Marine Jurassic

Pelecypods from

Central and Southern

Utah

GEOLOGICAL SURVEY PROFESSIONAL PAPER 483-C

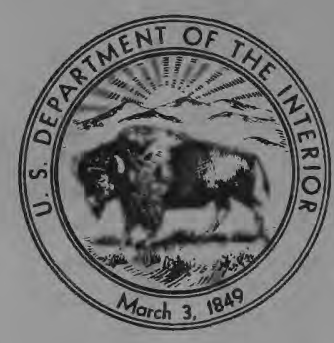



Marine Jurassic

Pelecypods from

Central and Southern

Utah

By RALPH W. IMLAY

C O N T R I B U T I O N S T O PA L E O N T O L O G Y

GEOLOGICAL SURVEY PROFESSIONAL PAPER 483-C

Descriptions and illustrations of

marine pelecypods of Jurassic age

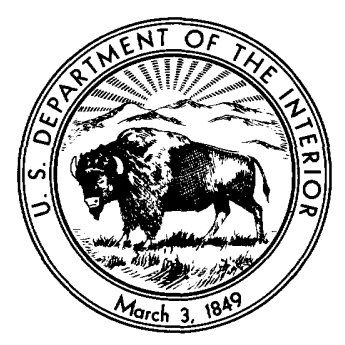

UNITED STATES GOVERNMENT PRINTING OFFICE, WASHINGTON : 1964 


\section{UNITED STATES DEPARTMENT OF THE INTERIOR STEWART L. UDALL, Secretary \\ GEOLOGICAL SURVEY \\ Thomas B. Nolan, Director}




\section{CONTENTS}

Abstract

Introduction.

Stratigraphic considerations

Ages and correlations.

Carmel Formation

Arapien Shale..................

\begin{tabular}{r|lr}
\hline $\begin{array}{r}\text { Page } \\
\text { C1 }\end{array}$ & Faunal associations and comparisons & $\begin{array}{r}\text { Page } \\
1\end{array}$ \\
1 & Geographic distribution & Summary of results \\
2 & Systematic descriptions & 8 \\
3 & Literature cited & \\
3 & Index &
\end{tabular}

\section{ILLUSTRATIONS}

[Plates follow index]

age 8 8 23 38

Plate 1. Nucula, Mytilus, Lopha, Idonearca?, Plicatula, Modiolus, Gervillia, Anomia?, Inoceramus, Ostrea (Liostrea), and Isognomon.

2. Camptonectes and Lima.

3. Trigonia, Vaugonia, Myophorella (Haidaia), and Myophorella (Promyophorella).

4. Astarte (Coelastarte), Quenstedtia, Myopholas, Pronoella, Cyprina?, Hamulus?, Cercomya, Thracia, Goniomya, and Pholadomya.

Page

FIGURE 1. Index map showing marine Jurassic localities in central and southern Utah.

\section{TABLES}

TABLES 1. Relative abundance of marine Jurassic pelecypods in central and southern Utah.

2. Regional distribution of marine Jurassic megafossils in central and southern Utah.

3-8. Geographic distribution of marine Jurassic megafossils in Utah.

3. Juab, San Pete, Piute, and Beaver Counties

4. Iron County

5. Washington County $---\frac{-1}{6}$

6. West side of San Rafael Swell, Emery County

7. East side of San Rafael Swell, Emery County

8. Wayne, Garfield, and Kane Counties. 



\title{
CONTRIBUTIONS TO PALEONTOLOGY
}

\section{MARINE JURASSIC PELECYPODS FROM GENTRAL AND SOUTHERN UTAH}

\author{
By RaLph W. IMLAY
}

\begin{abstract}
The pelecypod faunule in the marine Jurassic beds of central and southern Utah includes 31 genera and 44 species. The most common genera, in order of decreasing abundance, are Camptonectes, Ostrea, Pronoella, Vaugonia, Myophorella, and Lima. These genera include 80 percent of the specimens collected.

Pelecypods are more common than any other megafossils in the marine Jurassic rocks of central and southern Utah. They are most common in a belt about 40 miles wide that extends southwestward from the west side of the San Rafael Swell. Eastward they become less common as the limy unit wedges out into red siltstone of littoral origin. Westward the pelecypods become less common as the limy unit thickens greatly. To the west, deposition of calcareous muds was apparently too rapid for benthonic organisms to thrive. Nevertheless, all parts of the Jurassic sea were fairly shallow, as shown by the presence of such pelecypods as Ostrea, Lopha, Mytilus, Modiolus, Plicatula, and Isognomon, the absence of such pelecypods as Gryphaea and $\boldsymbol{N}_{\boldsymbol{1}}$ eleagrinella, and an absence of ammonites except at the northwest end of the San Rafael Swell.

Studies of the pelecypods from the limy lower unit of the Carmel Formation show that the lower part of that unit is of Middle Jurassic (Bajocian) age, that the entire unit is probably of Bajocian age, at least locally, and that the highest beds of that unit are not younger than earliest Late Jurassic (early Callovian). The unit is correlated with the lower part of the Twin Creek Limestone of northern Utah, western Wyoming, and southeastern Idaho.
\end{abstract}

The gypsiferous claystone and siltstone upper unit of the Carmel Formation has furnished a few fossils but only in southwestern Utah, and these belong to long-ranging species of Bajocian to Callovian age. This unit is considered to be of early Callovian age on the basis of stratigraphic position and of regional stratigraphic considerations.

The pelecypods from the Twelvemile Canyon Member of the Arapien Shale in central Utah show that it is the equivalent of the Twin Creek Limestone and of the Carmel Formation and ranges in age from Bajocian to early Callovian.

\section{INTRODUCTION}

This study of the marine Jurassic pelecypods from central and southern Utah is based on 176 collections made by 47 geologists. The oldest collection was made by C. D. Walcott in 1879 and the next oldest by T. W. Stanton in 1892 . All other collections have been made between 1907 and 1961. The study was undertaken at the request of J. C. Wright of the Geological Sur- vey as an aid in solving correlation problems concerning the formations and members of the San Rafael Group. The study does not include pelecypods from the Curtis Formation and equivalent beds.

Thanks are due to J. C. Wright for checking the geographic descriptions and locations of the collections from the Carmel Formation and for furnishing stratigraphic data concerning the Carmel Formation. C. T. Hardy kindly supplied data concerning collections from the Twelvemile Canyon Member of the Arapien Shale.

The index map of localities in central and southern Utah does not show the locations of many small creeks, washes, springs, and seeps that are mentioned in the locality descriptions. The positions of such geographic features may be found in the quadrangle maps of Utah and in the publications of Baker, Dane, and Reeside (1936); Gilluly (1929); Gregory (1950, 1951); Hardy (1952a, 1962); and Spieker (1946).

\section{BIOLOGIC ANALYSIS}

The marine Jurassic pelecypods from central and southern Utah that have been studied during preparation of this paper number about 1,700 specimens. Their distribution by genera and families is shown in table 1. The genera Camptonectes and Ostrea are by far the most common and could easily have been collected in much larger numbers. Vaugonia and Pronoella are a little less common but likewise represent a mere sampling of the numbers present in the outcrops. Nucula was found in abundance only near Cedar City and Thracia only at one place on Deep Creek north of Escalante. The number of specimens of the other genera, as shown in table 1 , represent all that have been obtained by many geologists during more than 60 years. The 17 genera and subgenera represented by $1-6$ specimens may be considered scarce. Eight genera and subgenera are represented by $14-37$ specimens and, therefore, are not very common. Only 8 genera and subgenera are represented by more than 64 specimens, and of these only 6 genera are widespread. 
Consequently most fossil collections from central and southern Utah are dominated by only a few genera, which in order of decreasing abundance include Camptonectes, Ostrea, Pronoella, Vaugonia, Myophorella (Promyophorella), and Lima (Plagiostoma).

TABLE 1.-Relative abundance of marine Jurassic pelecypods in central and southern Utah

\begin{tabular}{|c|c|c|}
\hline Family & Genus or subgenus & $\begin{array}{l}\text { Number of } \\
\text { specimens }\end{array}$ \\
\hline Nuculidae & Nucula & 100 \\
\hline $\begin{array}{l}\text { Parallelodontidae } \\
\text { Cucullaeidae.....- }\end{array}$ & Grammatodon? & 14 \\
\hline Mytilidae....... & Modiolus & 33 \\
\hline Isognomonidae.. & $\begin{array}{l}\text { Gervillia } \\
\text { Isognomon.. }\end{array}$ & $\begin{array}{r}4 \\
47 \\
37\end{array}$ \\
\hline Pinnidae-...- & $\begin{array}{l}\text { Inoceramus.-- } \\
\text { Pinna }\end{array}$ & \\
\hline $\begin{array}{l}\text { Pectinidae } \\
\text { Plicatulidae... }\end{array}$ & Camptonectes_- & $>450$ \\
\hline Limidae.....- & Llima (Plagiostoma) & $\begin{array}{l}18 \\
77\end{array}$ \\
\hline Ostreidae..... & $\begin{array}{l}\text { Ctenosireon } \\
\text { Ostrea (Liostrea) }\end{array}$ & $\begin{array}{r}3 \\
>300\end{array}$ \\
\hline Anomiidae.- & Anomia? & $\begin{array}{r}21 \\
5\end{array}$ \\
\hline Trigoniidae_. & Trigonia-- & $>210$ \\
\hline & Promyophorella & 90 \\
\hline Astartidae.... & Astarte & 1 \\
\hline Cyprinidae.. & \begin{tabular}{|l} 
Coelastarte \\
Pronoella
\end{tabular} & $\begin{aligned} & 30 \\
> & 230\end{aligned}$ \\
\hline $\begin{array}{l}\text { Lucinidae } \\
\text { Tancrediidae }\end{array}$ & $\begin{array}{l}\text { Lucina } \\
\text { Tancredia? }\end{array}$ & $\frac{4}{3}$ \\
\hline Quenstedtida & Ouenstedtia-. & \\
\hline Pleuromy & Pleuromya.............. & 6 \\
\hline Pholadomyida & $\begin{array}{l}\text { Pholadomya } \\
\text { Goniomya }\end{array}$ & $\begin{array}{r}31 \\
2\end{array}$ \\
\hline Pholadidae-_-_. & $\begin{array}{l}\text { Mactromya-...... } \\
\text { Myopholas }\end{array}$ & ${ }_{2}^{6}$ \\
\hline $\begin{array}{l}\text { Laternulidae......... } \\
\text { Thraciidae }\end{array}$ & Cercomya & \\
\hline Thraciidae.... & Thracia_-_-_---- & 64 \\
\hline
\end{tabular}

Other megafossils are less common than pelecypods in the marine Jurassic beds of central and southern Utah. Gastropods are about half as common but are fairly widespread. Only four ammonites have been found, and all these are from the northwestern part of the San Rafael Swell. Crinoid columnals have been obtained from 41 localities in various parts of central and southwest Utah, but only one occurrence is known in Emery County. Echinoid fragments have been obtained at eight localities in San Pete, Beaver, Washington, Iron, and Garfield Counties. One starfish has been found near Teasdale in Wayne County. The worm tube Hamulus? subquadratus (Meek and Hayden) (pl. 4, fig. 26) has been obtained at three localities in Emery County. Other less distinctive worm tubes are known from Kane and Beaver Counties. Some colonial corals, probably including Astrocoenia, have been found in Garfield, Kane, and Iron Counties.

\section{STRATIGRAPHIC CONSIDERATIONS}

The fossils described herein were obtained from the lower limy unit of the Carmel Formation of eastcentral, south-central, and southwestern Utah and from the Arapien Shale of central Utah. The overall characteristics and distribution of the Carmel Forma- tion have been described by Baker, Dane, and Reeside (1936, p. 6, 45, 54, 58) and have been summarized recently by Wright and Dickey $(1958$, p. 174, 175; 1963). The characteristics and distribution of the Arapien Shale have been described by Spieker (1946, p. 123125), Hardy (1952a), Hardy and Zeller (1953, p. 12641266), and Willard and Callaghan (1962).

The Carmel Formation, as discussed by Wright and Dickey $(1958$, p. 174), is readily divisible into two major units over its entire area of distribution. The lower unit consists mostly of gray limestone and shale. The upper unit consists of claystone, siltstone, and gypsum but locally in southern Utah contains considerable sandstone. It is generally red in its eastern areas of exposure and generally green to gray in its western areas. Westward the Carmel Formation thickens considerably (Wright and Dickey, 1958, p. 175) from 0 to at least 700 feet. In its westernmost exposures in Iron and Washington Counties it shows great resemblances lithologically to the Arapien Shale of central Utah and to the Twin Creek Limestone of north-central Utah. These resemblances probably are the basis for Spieker's (1946, p. 123) suggestion that the Arapien Shale may be recognized over a large area in southern and southwestern Utah.

In general, the Carmel Formation shows many of the same changes westward as are shown by equivalent beds exposed along the south side of the Uinta Mountains (Imlay, 1953, p. 58). The Carmel Formation along the east side of the San Rafael Swell consists of about 130 feet of sparsely fossiliferous limestone and silty limestone in the lower unit and about 175 feet of red siltstone containing some gypsum in the upper unit. It is comparable with the Carmel Formation exposed between White Rocks Canyon and Vernal in the Uinta Mountains.

The Carmel Formation on the west side of the San Rafael Swell consists of 135-200 feet of moderately fossiliferous oolitic limestone and interbedded clastics in the lower unit and 250-450 feet of greenish-gray clayey siltstone containing interbedded gypsum in the upper unit. It is comparable with the Carmel Formation exposed between Lake Fork and White Rocks Canyon in the Uinta Mountains.

The Carmel Formation exposed near Teasdale in Wayne County, on Deep Creek and Antimony Creek in Garfield County, and in western Kane County consists of 250-550 feet of moderately fossiliferous limestone and limy shale in the lower unit and 400-550 feet of clastic rocks containing interbedded gypsum in the upper unit. The lower unit shows resemblances to the Twin Creek Limestone exposed between Lake Fork and Duchesne River in the Uinta Mountains. 
Finally, the lower part of the Carmel Formation in Iron and Washington Counties consists of about 500-950 feet of red beds and shaly to medium-bedded limestone that show some resemblances to the lower part of the Twin Creek Limestone (members A-E) exposed near Peoa at the west end of the Uinta Mountains and at Monks Hollow in the Wasatch Range.

The Arapien Shale consists of a lower gray calcareous shale and limestone unit, named the Twelvemile Canyon Member, and an upper red siltstone and shale unit, named the Twist Gulch Member. The Arapien Shale is underlain by the Navajo Sandstone along Red Canyon about $2 \frac{1}{2}$ miles northeast of Nephi, Utah (Johnson, 1959 , p. 19, pl. 8) and along the west side of the Sevier Valley from 8 to 10 miles southwest of Marysvale, Utah (Willard and Callaghan, 1962). It is overlain by beds assigned questionably to the Morrison Formation in Salina Canyon east of Salina (Spieker, 1946, p. 125; Hardy, 1952a, p. 23, 76-78) and along the southeastern part of the Gunnison Plateau (Hardy, 1962, p. 54). In Salina Canyon, however, 177 feet of beds in the upper part of the Twist Gulch Member of the Arapien Shale consists of fossiliferous glauconitic sandstone, grit, and shale that lithologically resembles the Curtis Formation (Hardy, 1952a, includes units $31-35$ on p. 78) and contains the pelecypod Meleagrinella curta (Hall) which is common in the Curtis Formation of Utah and which has not been found elsewhere in any part of the Arapien Shale or in the Carmel Formation. For lithologic and faunal reasons, therefore, the 177 feet of beds in question in Salina Canyon is herein considered to be a western extension of the Curtis Formation.

The remainder of the Twist Gulch Member of the Arapien Shale consists mostly of unfossiliferous red siltstone and sandstone and attains thicknesses ranging from 1,265 feet to at least 1,910 feet (Hardy, 1952a, p. $23 ; 1952$ b, p. $94 ; 1962$, p. 53 ). It is lithologically similar to and occupies the same stratigraphic position as the Preuss Sandstone of north-central Utah (Baker, 1947; Imlay, 1952a, p. 1736-1739) and the Entrada Sandstone of central and southern Utah (Hardy, 1952a, p. 27, 28; Hardy and Zeller, 1953, p. 1266; Imlay, 1952 b, p. 1736).

The Twelvemile Canyon Member of the Arapien Shale is divisible lithologically into two parts. The lower part consists mostly of thin- to thick-bedded limestone that is locally oolitic but includes some shale. It is about a thousand feet thick southwest of Marysvale (Willard and Callaghan, 1962) and at least 800 feet thick in the northern part of the Gunnison Plateau (Hardy, 1952a, p. 16, 18, 19; Hardy and Zeller, 1953 p. 1264; Johnson, 1959, p. 23, 24). The upper part consists of gray calcareous shale and siltstone that is in part gypsiferous and red, locally includes salt beds at its top, and is commonly much contorted. This upper part is about 1,140 feet thick southwest of Marysvale (Willard and Callaghan, 1962). It is some thousands of feet thick on the west side of the Wasatch Plateau and near the Gunnison Plateau (Spieker, 1946, p. 12.5; Hardy, 1952a, p. 16, 17; Johnson, 1959, p. 22, 23, 24, 41; Hardy, 1962, p. 53), but the thicknesses cannot be determined because of complex distortion of beds. Estimates of thicknesses for the entire member in the Wasatch and Gunnison Plateaus areas range from 4,400 to 10,000 feet (Johnson, 1959, p. 22).

\section{AGES AND CORRELATIONS CARMEL FORMATION}

The age of the limy lower unit of the Carmel Formation, based on the pelecypods described herein, is Middle Jurassic to possibly early Late Jurassic. The Middle Jurassic age is shown by the presence in the lower part of the Carmel Formation of certain species of pelecypods that are known elsewhere in the western interior region only in beds that contain middle to upper Bajocian ammonites, such as Stemmatoceras, Stephanoceras, Zemistephanus, and Chondroceras. These pelecypods include the following:

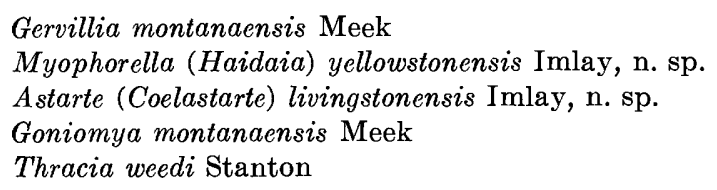

Most of these species have been obtained only in the lower fourth of the limy lower unit of the Carmel Formation at positions ranging from 15 to 30 feet above the top of the Navajo Sandstone. Thracia weedi Stanton has been obtained, however, throughout most of the limy lower unit. For example, at Deep Creek north of Escalante it occurs only 12-15 feet above the Navajo Sandstone (Mesozoic locs. 25671, 28468). At Mount Carmel it occurs from 20 to 30 feet below the top of the limy unit (Mesozoic locs. 28494, 28495). Near Pintura in Washington County it occurs 75 feet below the top of the 520-foot thick limy unit (Mesozoic loc. 27466). Evidently, if Thracia weedi Stanton is indicative of the Middle Jurassic, then essentially all of the limy unit is of that age.

Most of the pelecypods found in the limy lower unit of the Carmel Formation belong to species, or are closely similar to species, that elsewhere in the western interior region occur with ammonites of Bajocian age but also range higher into beds containing ammonites of early 
Callovian age, such as Cadoceras and Kepplerites. These pelecypods include the following:

Modiolus subimbricatus Meek

Mytilus of. $M$. whitei (Whitfield)

Pinna kingi Meek

Isognomon cf. $I$. perplana (Whitfield)

Camptonectes stygius White platessiformis White

Lima (Plagiostoma) occidentalis Hall and Whitfield

Ostrea (Liostrea) strigilecula White

Trigonia americana Meek elegantissima Meek

Vaugonia conradi (Meek and Hayden)

Myophorella (Promyophorella) montanaensis (Meek)

Astarte cf. A. meeki Stanton

Pronoella ef. P. cinnabarensis Stanton

Pleuromya subcompressa (Meek)

Pholadomya kingi Meek inaequiplicata Stanton

Cercomya punctata (Stanton)

The species of pelecypods in the preceding list have not been collected from all parts of the limy lower unit of the Carmel Formation (table 2). Four have been collected only from the lower part of that unit, four from both lower and middle parts, two from both middle and upper parts, and seven from all parts. The species that range throughout the unit are represented by many specimens. The species that have been found only in the lower part of the unit are, with one exception, represented only by a few specimens. Most of the other species listed are represented by a moderate number of specimens.

These relationships suggest that the apparent stratigraphic ranges of these pelecypod species within the limy lower unit may not be the real ranges but may reflect some environmental factor that influenced their development or some depositional factor that controlled their preservation after burial. Their stratigraphic distribution within the unit appears, therefore, to show only that the upper part of the unit cannot be younger than early Callovian and is not necessarily younger than Bajocian.

A Middle Jurassic age for the limy lower unit is supported by other organisms, including corals, nerineid gastropods, and ammonites. Of these, corals have been collected at eight localities from the lower and middle parts of the unit. They are all poorly preserved but belong in part to the genus Astrocoenia, which in Montana and Wyoming has been found only in beds of Bajocian age (Wells, 1942; Imlay, 1956b, p. $577,578,583,584)$. The only other recorded occurrences of corals in the Jurassic of the western interior are (1) in southeastern Idaho in the Wolverine Canyon Limestone Member of the Preuss Sandstone (Imlay, 1952a, p. 1740-1742) and (2) in southwestern Montana as pebbles in the basal part of the Stump
TABLE 2.-Regional and stratigraphic distribution of marine Jurassic megafossils in central and southern Utah

[ $\times$ indicates that stratigraphic position is unknown]

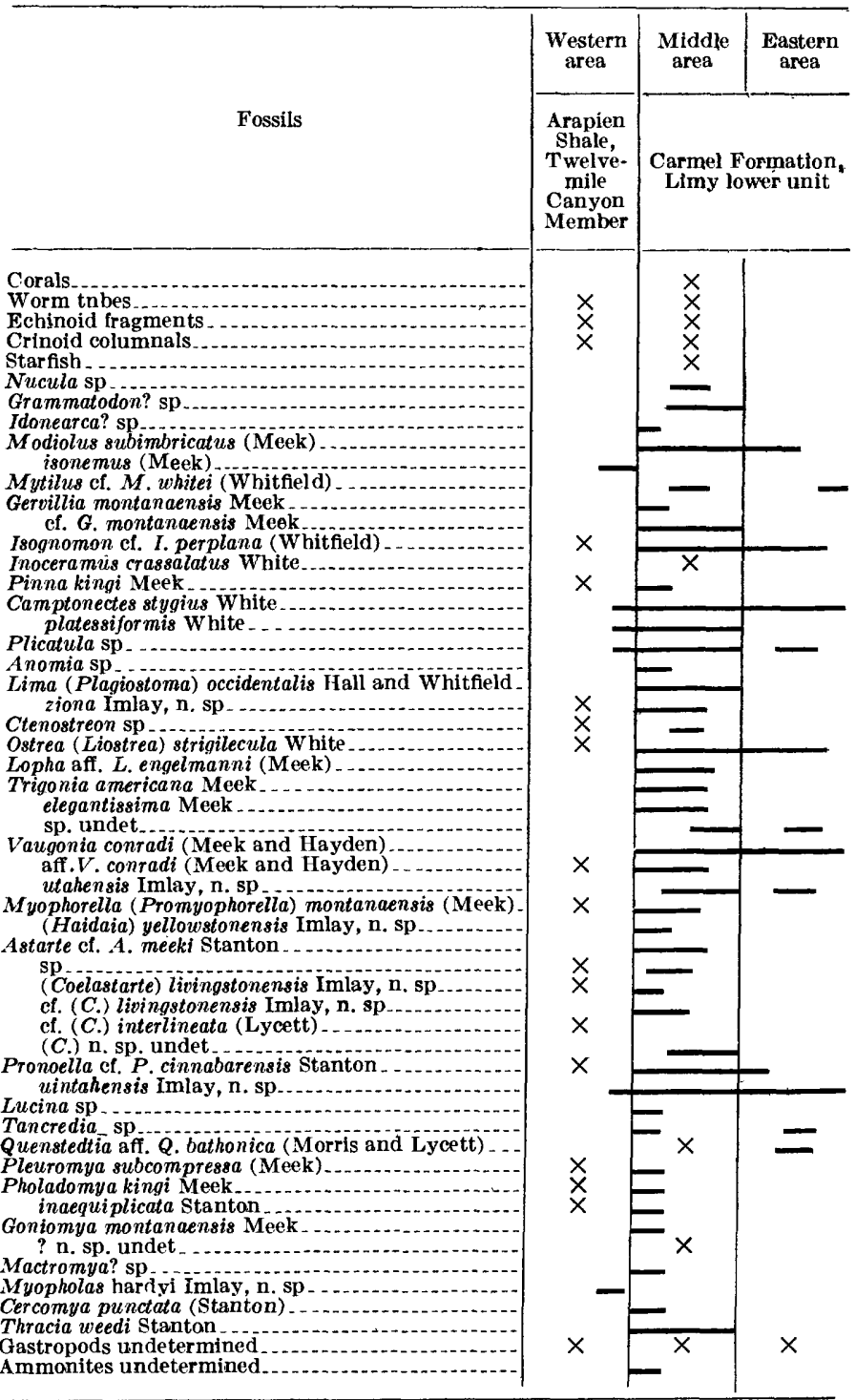

Sandstone exposed in the Centennial Range. The corals in the Stump Sandstone were derived from older Jurassic beds of unknown age, but the corals in the Preuss Sandstone are evidence that corals existed in the western interior sea after Middle Jurassic time. Nevertheless, as most of the occurrences of corals in the western interior are in Middle Jurassic beds, their presence in the Carmel Formation favors a Middle Jurassic age for that part of the formation in which they occur.

The age value of the nerineid gastropods in the Carmel Formation is similar to that of the corals. In the writer's experience they are fairly common in Middle Jurassic beds in Wyoming and Idaho and are unknown in younger Jurassic beds, except for an occurrence in 
the Wolverine Canyon Limestone Member of the Preuss Sandstone in southeastern Idaho (1952a, p. 1742). By comparison the occurrence of such gastropods in moderate abundance in all parts of the limy lower unit of the Carmel Formation is suggestive of a Middle Jurassic age for that unit.

Somrewhat stronger evidence for the Bajocian age of the lower unit of the Carmel Formation is furnished by four poorly preserved ammonites (Mesozoic locs. $12555,20351,25678$ ) found in the lower $5-40$ feet of the formation in the northwestern part of the San Rafael Swell. These ammonites belong to the genus Zemistephanus which occurs elsewhere in beds of Bajocian age in the northern part of the western interior region in the Cook Inlet region, Alaska, and in the Queen Charlotte Islands, Alaska. The ammonites do not resemble any from beds of Callovian age in the same regions.

In summation, the limy lower unit of the Carmel Formation contains many pelecypod species that elsewhere in the western interior region occur only in beds of Bajocian to early Callovian age. In addition, the lower part of that unit contains a few pelecypod species and some ammonites that have been found elsewhere only in beds of Bajocian age. A Bajocian age for the upper part of the unit locally, as at Mount Carmel and near Pintura, is suggested by the presence of the pelecypod Thracia weedi Stanton, which has been found only in beds of Bajocian age. A Bajocian age for the entire lower unit is suggested likewise by the presence of corals and of nerineid gastropods, as those organisms have been found mostly in beds of Bajocian age elsewhere in the western interior. Evidently the fossil evidence is very strong for a Bajocian age for the lower part of the lower unit, favors a Bajocian age for the upper part of lower unit, and shows that no part of the unit is younger than early Callovian.

This general age assignment for the lower unit of the Carmel Formation is also favored by lithologic and stratigraphic resemblances to members of the Twin Creek Limestone of northern Utah (Imlay, 1953, p. 55-59, fig. 3). If correlations based on the comparison are apt, then the lowest beds of the limy lower unit of the Carmel in the San Rafael Swell should be of Bajocian age, and the highest limy beds of the lower unit should be of earliest Callovian age. A Callovian age for the highest limy beds cannot be proved, however, because those beds have furnished few fossils, and the pelecypods present belong to species that range from Bajocian into the Callovian.

Similarly, in southwestern Utah between Gunlock and Cedar City, the limy lower unit of the Carmel Formation above the basal Red beds resembles members B-D, or possibly E, of the Twin Creek Limestone as exposed in north-central Utah. There is no definitive lithologic or faunal evidence, however, that the upper part of this unit in southwest Utah correlates directly with members $\mathrm{D}$ or $\mathrm{E}$ of the Twin Creek. In fact, the presence of such pelecypods as Thracia weedi Stanton and Gervillia cf. G. montanaensis Meek near the top of the limy lower unit suggests that no part of that unit is younger than member $\mathrm{C}$ of the Twin Creek Limestone.

The above remarks concerning the age of the Carmel Formation apply only to the limy lower unit of the formations as defined by Wright and Dickey (1958, p. 174). The overlying unit of gypsiferous claystone and siltstone on the San Rafael Swell has furnished no fossils, but its age is considered to be early Callovian on the basis of stratigraphic position and of regional stratigraphic relations (Imlay, 1952b, p. 963). The approximately equivalent beds in southwestern Utah that Gregory (1950, p. $94-98$; 1951, p. 27-33) referred to the Entrada Sandstone, Curtis Formation, and Winsor Formation are now included in the upper part of the Carmel Formation (Wright and Dickey, 1963). Their age is probably Callovian on the basis of stratigraphic position. A few fossils have been found, however, in limy beds associated with gypsum that Gregory referred to his Curtis Formation (Mesozoic locs. 15498, 18867, 20353). These fossils include the pelecypods Ostrea, Modiolus, Pronoella?, and Camptonectes, a neritid gastropod, and the crinoid columnals named Pentacrinus asteriscus Meek and Hayden. These merely show that the beds are of Jurassic age.

\section{ARAPIEN SHALE}

All the fossils from the Arapien Shale that are described or listed herein are from the Twelvemile Canyon Member. The exact stratigraphic positions of many of the fossil localities in the Twelvemile Canyon Member are not well known because of structural and stratigraphic complications, because of the thousands of feet of beds involved, and because in many places the base of the formation is not exposed. The thin- to thick-bedded limestone in the lower part of the member has, however, furnished all the collections from southwest of Marysvale in Piute County and has probably furnished the collections from near Mineral Gap in Beaver County (table 3). In addition, some oolitic limestone from 250 to 450 feet above the base of the member exposed on the first ridge south of Red Canyon, $2 \frac{1}{2}$ miles northeast of Nephi, has furnished crinoid columnals, echinoid spines, Camptonectes, and Pinna (Johnson, 1959, p. $23,24,41$ ).

Most of the remainder of the collections listed in table 3 are from the calcareous shale and siltstone in the upper part of the Twelvemile Canyon Member. 
TABle 3.-Geographic distribution of marine Jurassic megafossils in Juab, San Pete, Piute, and Beaver Counties, Utah [Nos. 1-15 refer to numbers on fig. 1. All five-digit numbers refer to Geol. Survey Mesozoic locs.]

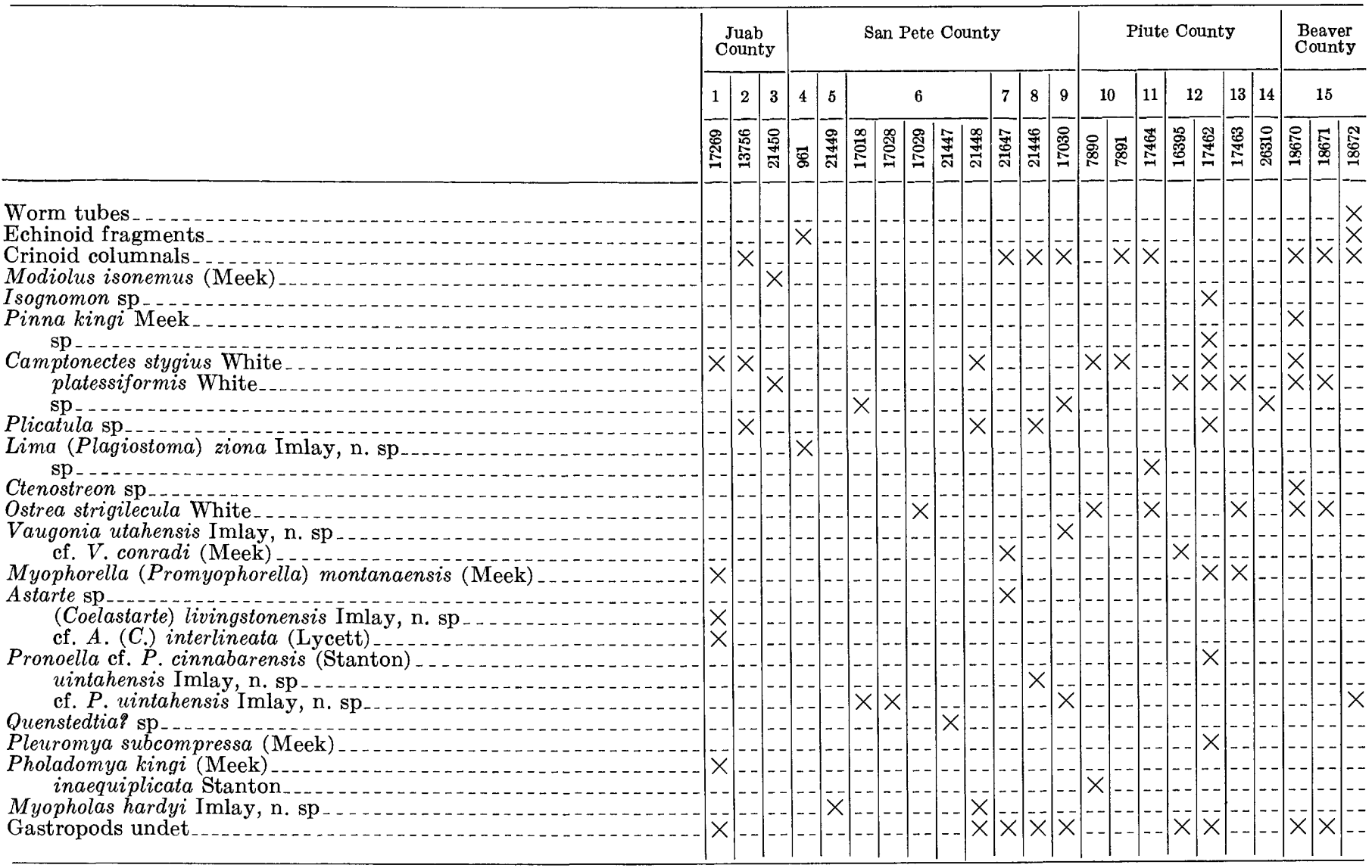

These include in particular the collections made by C. T. Hardy and E. M. Spieker and associates in areas near Mayfield and Gunnison. Four of these collections are labeled as probably from the upper 600 feet of the member (USGS Mesozoic locs. 21447-21450) and two as probably about 1,800 feet below its top (USGS Mesozoic locs. 21646, 21647).

Most of the fossils found in the Twelvemile Canyon Member of the Arapien Shale are identical specifically with those in the Twin Creek Limestone and in the limy lower unit of the Carmel Formation. The presence of Astarte (Coelastarte) livingstonensis Imlay, n. sp. (USGS Mesozoic loc. 17269) indicates that part of the Arapien Shale is as old as Bajocian because that species elsewhere in the western interior region has been found only in beds of that age. Most of the species found in the Twelvemile Canyon Member, however, range throughout most of the Twin Creek Limestone and by comparison could be as young as early Callovian or as old as Bajocian.

Stratigraphically, correlation of the Twelvemile Canyon Member of the Arapien Shale with the Twin Creek Limestone seems reasonable, considering that both are underlain by the similar appearing Navajo, or Nugget Sandstone, and both are overlain by saltbearing red beds. Furthernore, the lower part of Twelvemile Canyon Member exposed on the south side of Red Canyon, northeast of Nephi, shows many resemblances to the lower part of the Twin Creek Limestone. Thus, the basal 50-80 feet of oolitic limestone of the Tweivemile Canyon Member exposed at and near the bottom of Red Canyon could correspond with Member B of the Twin Creek Limestone, the overlying 100200 feet of shaly limestone with Member $\mathrm{C}$, the ridgecapping 200 feet of oolitic to dense limestone beds with Members $\mathrm{D}$ and $\mathrm{E}$, and the overlying splintery limestone with Member F.

\section{FAUNAL ASSOCIATIONS AND COMPARISONS}

The distribution, abundance, and variety of marine megafossils in the Carmel Formation and Arapien Shale of central and southern Utah are related to a gradual northwestward thickening of the associated limestone and calcareous shale from 0 in southeastern Utah to as much as 700 feet in Washington County, 2,140 feet in Piute County, and at least 3,000 feet in San Pete and Juab counties. This northwestward increase in calcareous rocks reflects a change from 
littoral deposition on the east and southeast to shallow marine deposition on the northwest (Wright and Dickey, 1958, p. 174, 175) and to progressively more rapid subsidence of the sea floor, toward the northwest. Furthermore, the westernmost marine Jurassic calcareous rocks that are preserved are comparable in thickness and lithologic features to the Twin Creek Limestone of north-central Utah and adjoining areas (Imlay, 1950, 1953), are probably a southern extension of that limestone, and similarly appear to have been formed in the open sea at a considerable distance from its western shore.

The relations of these lithologic changes to the associated fossils may be illustrated by considering all fossil occurrences in three areas (table 2) that trend northeastward and eastward roughly parallel to the eastern and southern shoreline of the Jurassic sea and that are partly separated by uplifts and plateaus on which Jurassic rocks are not exposed. The eastern area, thus selected, includes all fossil-collecting localities on the eastern side of the San Rafael Swell in Emery County as well as a few localities in central and eastern Wayne County east of Capitol Reef. The middle area includes all fossil-collecting localities in Washington, Iron, and Kane Counties, in western Garfield County east of Antimony and north of Escalante, in western Wayne County near Teasdale and Bicknell, and on the western side of the San Rafael Swell in Emery County. The western area includes all fossil-collecting localities in Beaver, Piute, San Pete, and Juab Counties.

Inspection of the fossils listed in table 2 shows that all three areas contain typical shallow-water attached pelecypods such as Ostrea, Modiolus, Plicatula, and Isognomon. All three contain the bottom-dwelling pelecypods Vaugonia, Pronoella, and Quenstedtia and the free-swimming pelecypod Camptonectes, and all contain gastropods. The middle area contains the greatest variety of organisms and the eastern area the least. Such organisms as worm tubes, echinoids, and crinoids have been found only in the middle and western areas and corals only in the middle area. Ammonites have been found only at the northwest end of the San Rafael Swell and starfish only near Teasdale, both in the middle area.

Table 2 does not show, however, that decrease in variety away from the middle area is accompanied by a great decrease in the abundance of individuals of most of the species or that the decrease in abundance is particularly striking between the Jurassic beds exposed on the west side of the San Rafael Swell in Emery County and the Jurassic beds west of the Wasatch Plateau in San Pete, Juab, and Piute Counties. This westward change is quite comparable to that occurring in the Twin Creek Limestone between western Wyoming and southeastern Idaho (Imlay, 1957, p. 496, 497). In the Twin Creek Limestone, as in the Carmel Formation, the most fossiliferous beds occur where the formation is of moderate thickness and contains interbeds of thin-bedded limestone and calcareous shale. Both formations become less fossiliferous northwestward as they thicken and as thin beds become less common. Both formations contain such shallow-water pelecypods as Ostrea, which shows that the relatively less fossiliferous thicker sequences to the west were not deposited in deep water.

The Jurassic megafossils in the Carmel Formation include many species in common with the Twin Creek Limestone of north-central Utah, southeastern Idaho, and western Wyoming, and with equivalent beds in the Yellowstone National Park area of Montana and Wyoming (Stanton, 1899, p. 600-640; Imlay, 1956a; 1956 b, p. $576,577,583,584,591)$. The most striking difference is the absence in the Carmel Formation of the pelecypod genera Gryphaea and Meleagrinella and a near absence of ammonites, except in one small area along the northwest side of the San Rafael Swell. The presence of a fair number of gastropods suggests faunal comparisons with the lower part of the Twin Creek Limestone and with the Gypsum Spring Formation of north-central Wyoming. The presence of corals suggests comparisons with the Middle Jurassic assemblages in north-central Wyoming and south-central Montana (Imlay, 1956b, p. 577, 578, 583, 584).

The few Jurassic megafossils that have been obtained from the Arapien Shale are mostly from its upper part and most belong to species that occur in the Twin Creek Limestone and in the Carmel Formation. The shale may have been deposited in slightly deeper water than the Carmel Formation, but the presence of such pelecypods as Ostrea and Modiolus indicate that the water was fairly shallow.

In summation, the megafossils of the Jurassic formations in central and southern Utah are closely similar to those in beds of Middle Jurassic age in southeastern Idaho, southwestern Wyoming, and southern Montana. They occur in greatest variety and numbers in a middle area or belt about 45 miles wide extending southwestward from the west side of the San Rafael Swell roughly parallel to a former shoreline that lay to the southeast and south. In this middle area the Jurassic calcareous sequence is of moderate thickness and consists mostly of thin-bedded limestone interbedded with calcareous shale. Southeastward, fossils become less common as the calcareous sequence wedges out into red siltstone and claystone of probable littoral origin (Wright and Dickey, 1958, p. 174). Northwestward, fossils become 
less common as the calcareous sequence thickens enormously, presumably owing to rapid subsidence accompanied by equally rapid sedimentation. Apparently both southeastward and northwestward from the middle area, conditions on the sea bottom became less favorable for benthonic organisms.

\section{GEOGRAPHIC DISTRIBUTION}

The occurrences by county and locality of the fossils described herein are shown in tables 3-8. The general position of each locality is shown in figure 1. Detailed descriptions of each locality are given in the following table.

TABLE 4.-Geographic distribution of marine Jurassic megafossils in Iron County, Utah

[Nos. 16-25 refer to numbers on fig. 1. All five-digit numbers refer to Geol. Survey Mesozoic locs.]

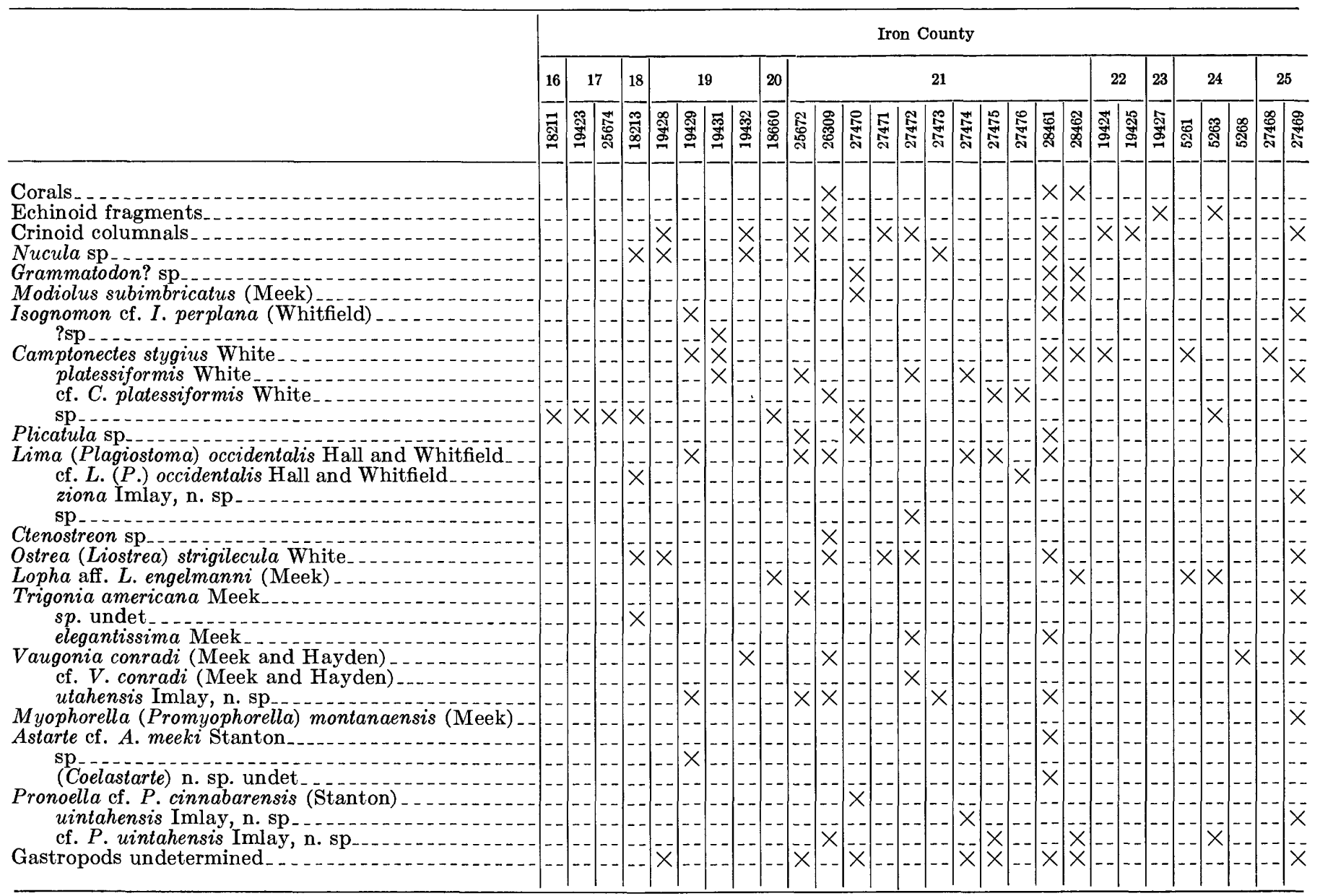


TABLE 5.-Geographic distribution of marine Jurassic megafossils in Washington County, Utah [Nos. 26-42 refer to numbers on fig. 1. All five-digit numbers refer to Geol. Survey Mesozoic locs.]

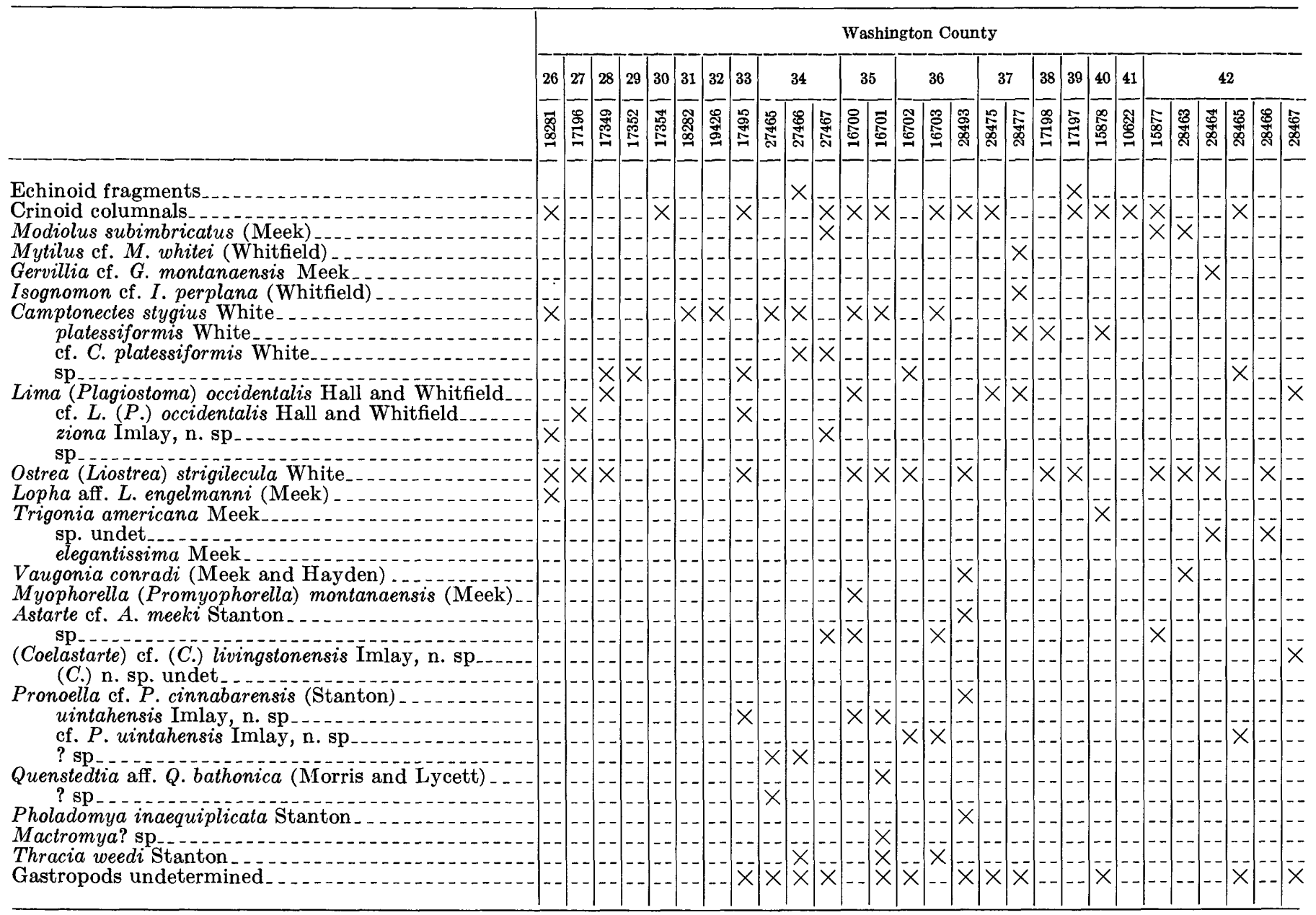




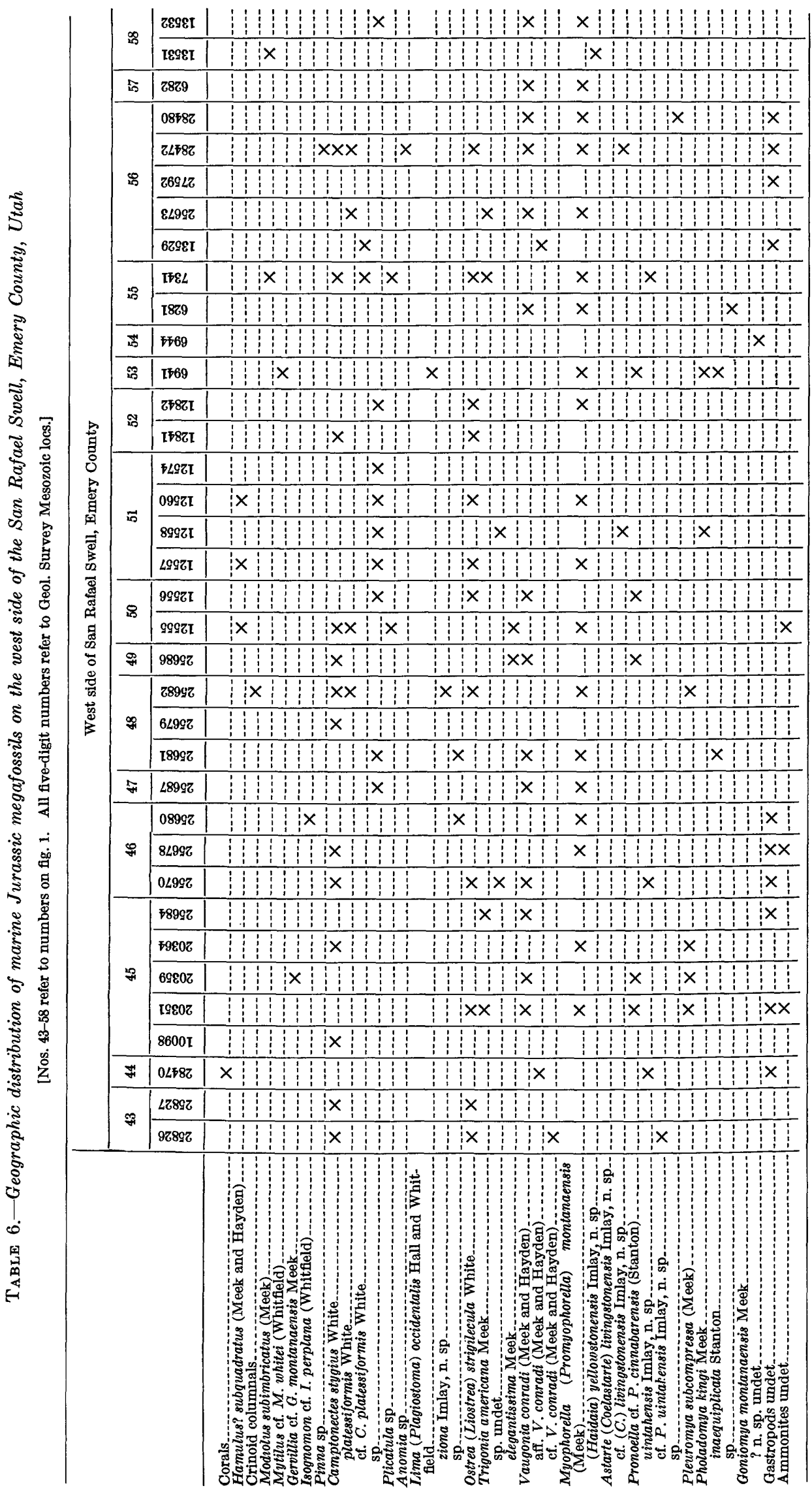


PELECYPODS FROM CENTRAL AND SOUTHERN UTAH

TABLE 7.-Geographic distribution of marine Jurassic megafossils on the east side of the San Rafael Swell in Emery County, Utah [Nos. 59-71 refer to numbers on fig. 1. All five digit numbers refer to Geol. Survey Mesozoic locs.]

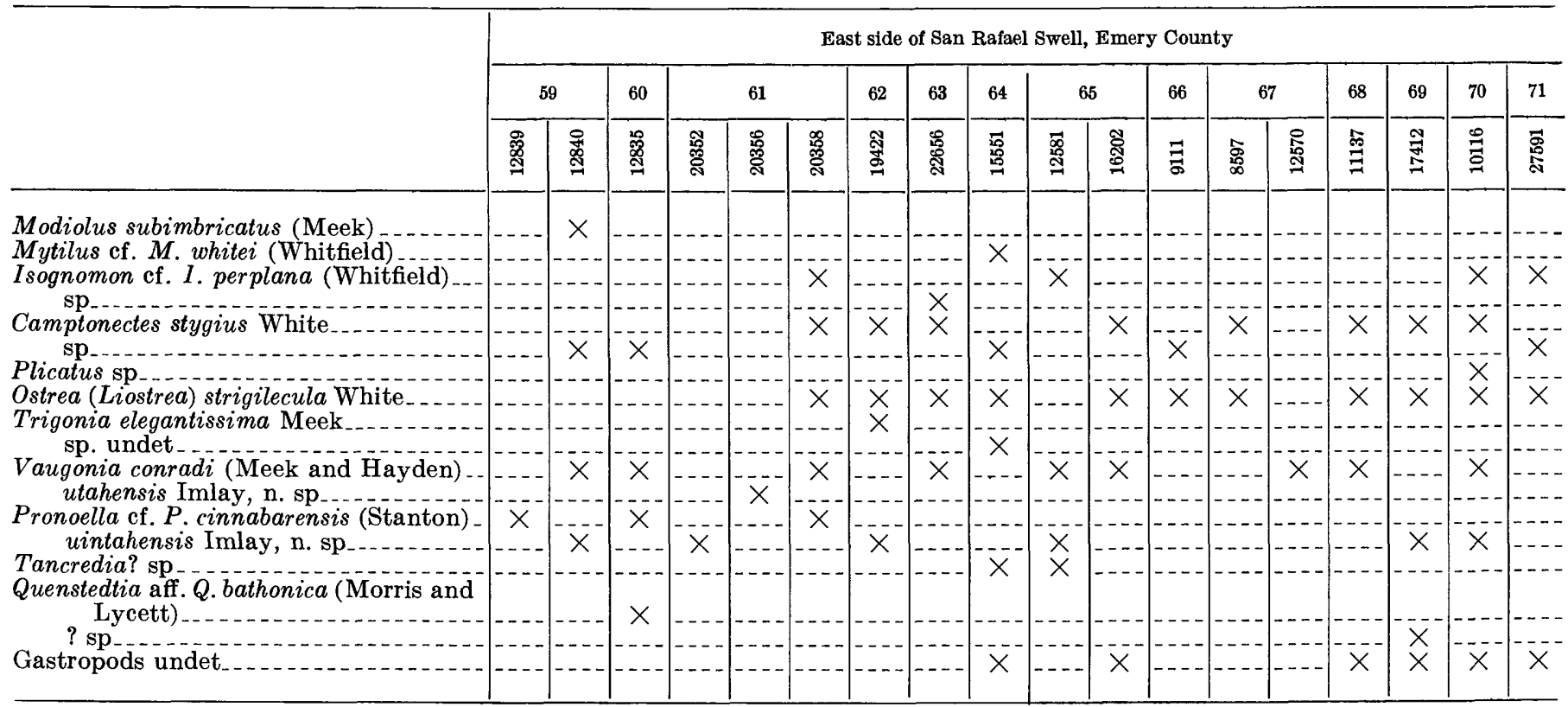


TABLE 8.-Geographic distribution of marine Jurassic [Nos. 72-94 refer to numbers on fig. 1. All five-

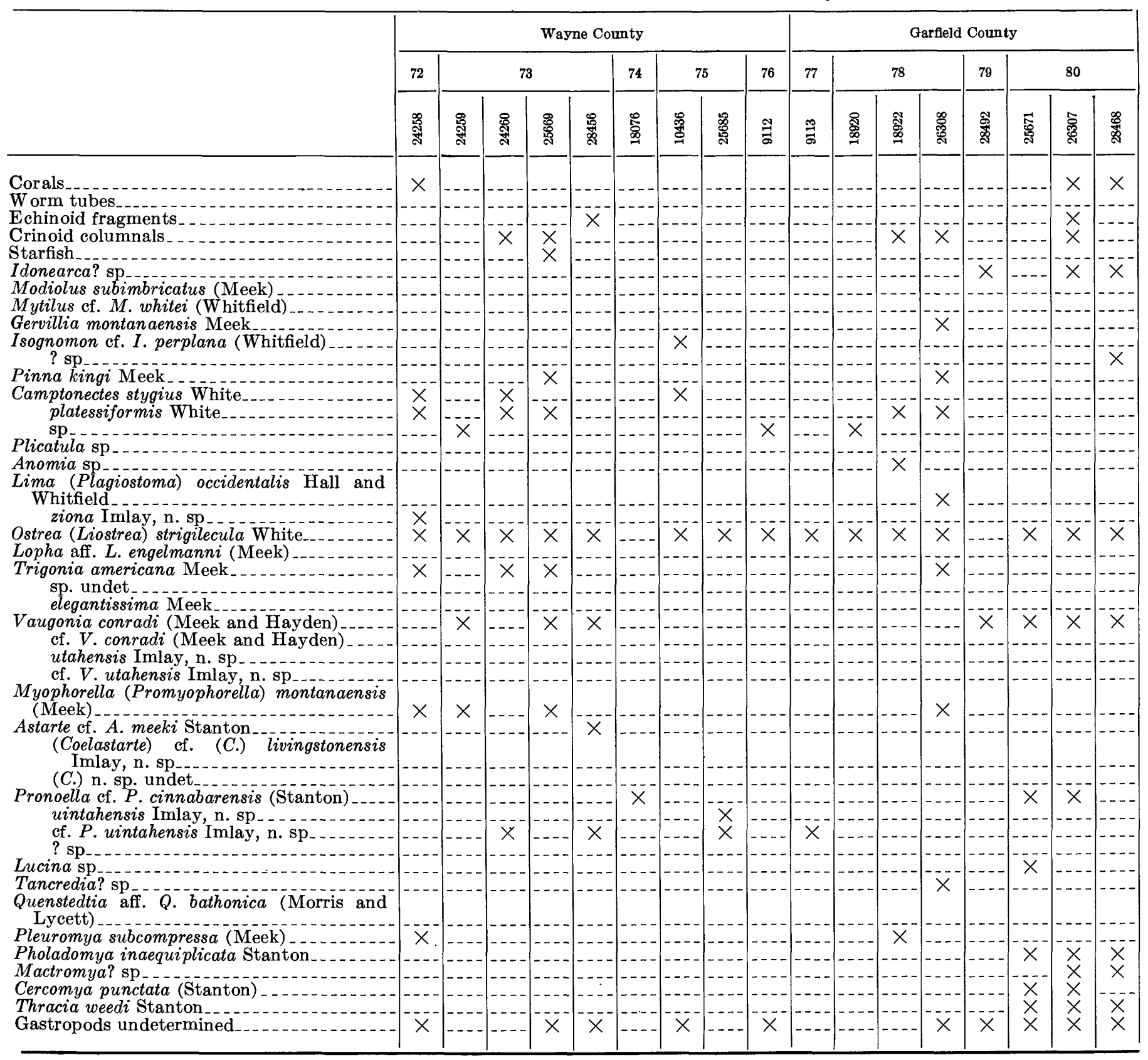


megafossils in Wayne, Garfield, and Kane Counties, Utah

diget numbers refer to Geol. Survey Mesozoic locs.]

\begin{tabular}{|c|c|c|c|c|c|c|c|c|c|c|c|c|c|c|c|c|c|c|c|c|c|c|c|c|c|c|}
\hline & & & & & & & & & & & & & Kane & Count & & & & & & & & & & & & \\
\hline 81 & 82 & & & 83 & & 84 & 85 & & 86 & & & & & 87 & & & & 88 & & 89 & & 90 & 91 & 92 & 93 & 94 \\
\hline $\begin{array}{l}\stackrel{0}{0} \\
\text { s. }\end{array}$ & 戀 & 岕 & 鹃 & 㑾 & 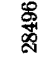 & : & 悹 & 胥 & $\begin{array}{l}\text { 笣 } \\
\text { 量 }\end{array}$ & $\begin{array}{l}\text { 密 } \\
\text { 恶 }\end{array}$ & 鴗 & 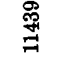 & 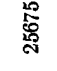 & $\begin{array}{l}\text { : } \\
\text { 怘 }\end{array}$ & 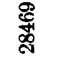 & 胥 & 荢 & $\begin{array}{l}\text { 咆 } \\
\text { 盗 }\end{array}$ & 古 & 茦 & 惫 & 罯 & 营 & 落 & 丞 & 䓌 \\
\hline- & & $\begin{array}{l}-- \\
--\end{array}$ & $\stackrel{x}{x}$ & $\underset{x}{x}$ & 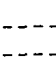 & & & & $\ldots$ & & & & & & & & & & & & & & & & & \\
\hline & & $x$ & $x$ & $\begin{array}{l}-1- \\
--1\end{array}$ & - & - & $\ldots$ & - & $x$ & $\ldots$ & $\cdots$ & $x$ & & & & & & & & & & & & & & $-1--$ \\
\hline & & 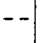 & - & $\cdots$ & $\cdots$ & & $-\cdots$ & $\cdots$ & 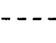 & $\cdots$ & -- & $\cdots$ & & & & & & & & & & & & & 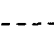 & $\mid-\ldots$ \\
\hline - - & {$[--$} & $\bar{x}$ & $x$ & $\cdots$ & $\cdots$ & $x$ & $\cdots$ & {$[-\cdots$} & $\cdots$ & 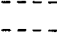 & $x$ & $x$ &.- & $\ldots$ & & & & & & & $\ldots$ & & $\ldots$ & $x$ & $\bar{x}$ & $\mid---1$ \\
\hline & & $=-1$ & 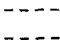 & -1 & & & |-. & $\ldots$ & -- & $\cdots$ & $-\ldots$ & $-\ldots$ & & $\cdots$ & & & & & & & & & & & $--\cdot$ & $-\cdots$ \\
\hline- & & - & $x$ & $x$ & $x$ & $x$ & $-\cdots$ & $\cdots$ & $\ldots$ & 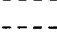 & 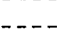 & $\cdots$ & $\cdots$ & $\cdots$ & $x$ &.- & & & & & & & -- & $x$ & $\cdots$ & $-1-1$ \\
\hline$\cdots$ & & -- & 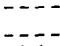 & {$[--$} & 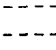 & - & & {$[--1$} & . & 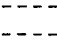 & & 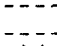 & 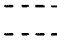 & 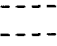 & 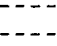 & & & & & & & & & -1 & & - \\
\hline-- & & $\begin{array}{l}x \\
x\end{array}$ & $\stackrel{x}{x}$ & {$[--$} & $\cdots$ & $\begin{array}{l}x \\
x\end{array}$ & $\mid---$ & $\mid--1]$ & $\cdots$ & $-\cdots$ & $\stackrel{x}{x}$ & X & $-\bar{x}$ & $\cdots$ & $\cdots$ & 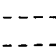 & $\cdots$ & $x$ & $\cdots$ & $x$ & $\times$ & $x$ & $\cdots$ & $\stackrel{\times}{x}$ & $\times$ & $\times$ \\
\hline & & -- & $\cdots$ & $-\cdots$ & $\cdots$ & $\cdots$ & $\ldots$ & $-\cdots$ & $\cdots$ & $\cdots$ & $\cdots$ & $\cdots$ & $\cdots$ & $\ldots$ & $\times$ & $\cdots$ & $\times$ & $\cdots$ & $\times$ & . & $-\bar{x}$ & 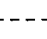 & & 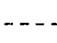 & $\ldots$ & $-\cdots$ \\
\hline & & -1 & & & & $\ldots$ & - & & & & $\ldots$ & $\ldots$ & $\ldots$ & $\ldots$ & 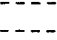 & . & & -1 & & & & 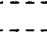 & & & 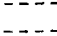 & $1-1$ \\
\hline & & - & $x$ & $\ldots$ & $\ldots$ & $\ldots$ & $x$ & $y$ & & & & & & & & $Y$ & & & & & & $x$ & & & & - \\
\hline-- & $x$ & $\bar{x} \mid$ & $x$ & $-\ldots$ & $\cdots$ & $\bar{x}$ & $\bar{x}$ & . & - & $x$ & $x$ & $-\bar{x}$ & $\hat{x}$ & 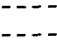 & $\cdots$ & $\lambda$ & 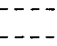 & & & & - & & & $\cdots$ & $x$ & $\cdots$ \\
\hline & & & $x^{-}$ & $\ldots$ & {$[-1$.} & $\cdots-$ & $\ldots$ & _. & -2 & - & 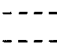 & $-\cdots$ & 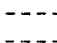 & -3 & $\cdots$ & 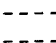 & - & $x$ & & $x$ & - & & & $-\cdots$ & - & 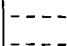 \\
\hline & & & 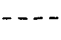 & $\ldots$ & -- & $\cdots$ & - & -- & $\cdots$ & -- & $\cdots$ & $-\bar{x}$ & $\times$ & $\cdots$ & $\cdots$ & - &.- & $\cdots$ & & $\ldots$ & -- &.- & & - & $\cdots$ & $-\cdots$ \\
\hline & & & $\ldots$ & $x$ & $\ldots$ & $\ldots$ & 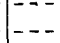 & -- & $\ldots$ & --- & 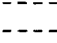 & त & 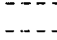 & $-\ldots$ & - & 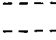 & 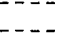 & 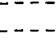 & & $\cdots$ &.- & & & - & $\cdots$ & $\mid$ \\
\hline & & 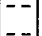 & $x$ & 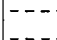 & - & $x$ & 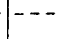 & & $\cdots$ & 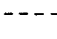 & & & & $\cdots$ & $\cdots$ & $\ldots$ & $\cdots$ & $\bar{x}$ & & . & - & . & - & $\cdots$ & $\times$ & $\bar{x}$ \\
\hline & & & & & & & -1 & & $\cdots$ & $\cdots$ & 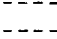 & $x$ & $-\cdots$ & $x$ & 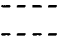 & 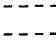 & $\cdots$ & $x$ & & $\cdots$ & $\ldots$ & $\ldots$ & 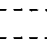 & $\cdots$ & & $x$ \\
\hline & & $x$ & & 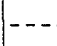 & $\ldots$ &. & $\ldots$ & & & & & & & . & 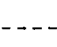 & $\cdots$ & $x$ & $z_{1}$ & & & 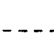 & & & & & - \\
\hline & & & & 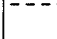 & & & & & - & & & $\lambda$ & & $\cdots$ & $\ldots$ & 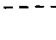 & & & & & & & & & & 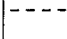 \\
\hline & & & & & & & & & & & $x$ & & & & & & & & & $x$ & $x$ & & & & & -- \\
\hline & & $\bar{x}$ & $\underset{\times}{X}$ & & & X & - & & -- & & $-\cdots$ & Y & & & $y$ & & $\cdots$ & $\cdots$ & & Y & $Y$ & . & & & $y$ & 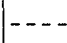 \\
\hline & & & $x$ & $\bar{x}$ & & $x$ & - & & $\cdots$ & $x$ & $\cdots$ & $x$ & {$\left[\begin{array}{ll}-\cdots \\
-\cdots\end{array}\right.$} & $x$ & $\lambda$ & $-\cdots$ & $x$ & 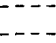 & $\cdots$ & $x$ & $x$ & 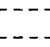 & $x$ & $\bar{x}$ & $x$ & $\bar{x}$ \\
\hline & & & & 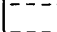 & $x$ & $\bar{x}$ & - & & - & $\cdots$ & $\cdots$ & & & $\cdots$ & $\cdots$ & .... & $\cdots$ & . & & $\ldots$ & -- & . & . & $\cdots$ & $\cdots$ & $\cdots$ \\
\hline & & & & & & & & & & & & & & & & & & & & & & & & & & \\
\hline & & & & & & & & & & & & & & & & & & & & & & & & & & $\times$ \\
\hline & & & & & & & & & & & & & & & & & & & & & & & & & & 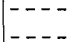 \\
\hline & & & & & & & & & & & & & & & & & & & & & & & & & & $\ldots$ \\
\hline-1 & & & & & & & & & & & & & & & & & & & & $x$ & $x$ & & & & & \\
\hline$x$ & & & $x$ & $x$ & $x$ & $x$ & & & - & $x$ & & & & & & & $x$ & $x$ & & $\hat{x}$ & $\hat{x}$ & $\bar{x}$ & $x$ & $x$ & & $x$ \\
\hline
\end{tabular}




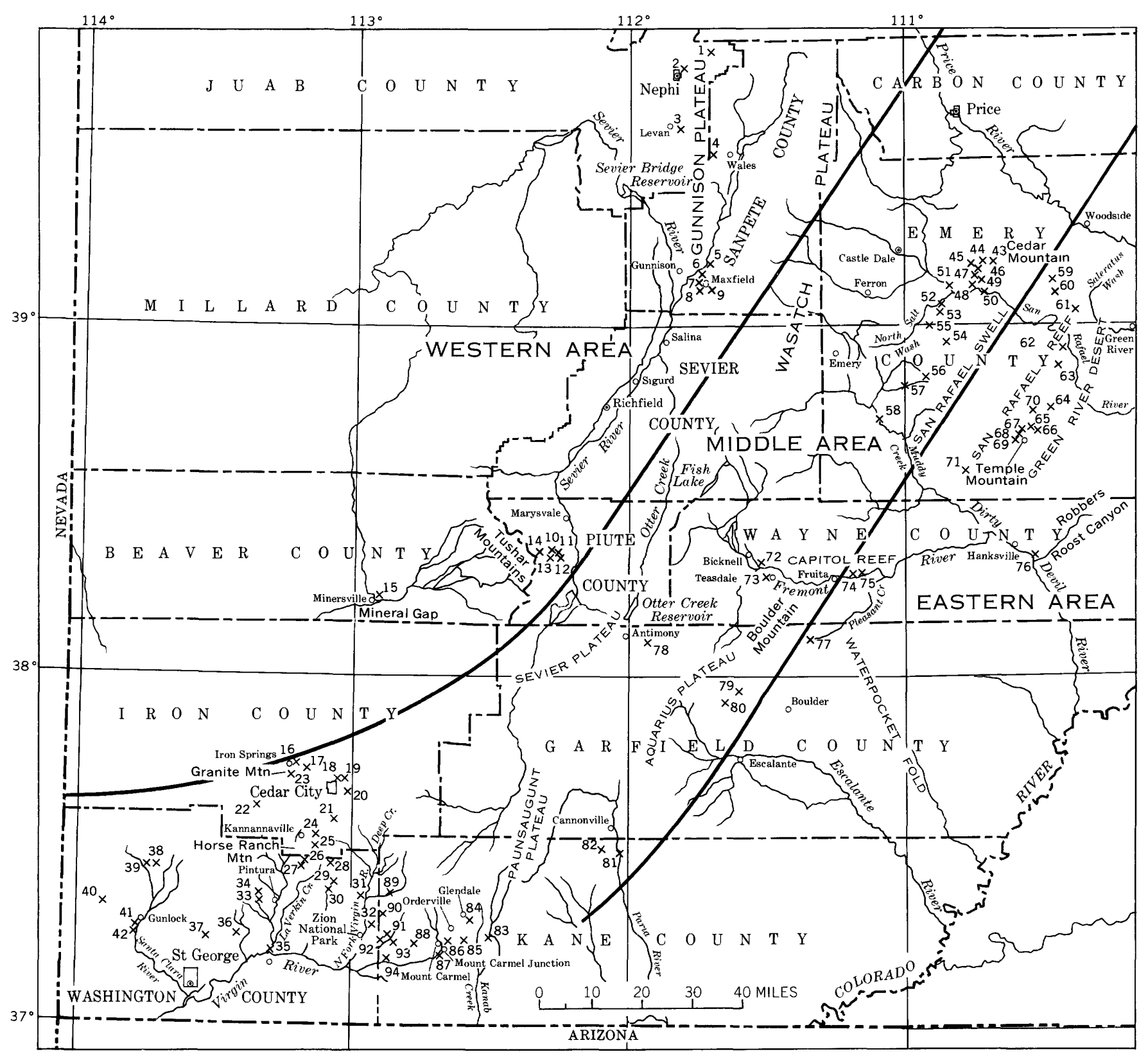

FIGURE 1.-Index map showing marine Jurassic localities in central and southern Utah. (Numbers on map refer to those given in tables 3-8.) 
Localities at which marine megafossils of Jurassic age have been collected in central and southern Utah

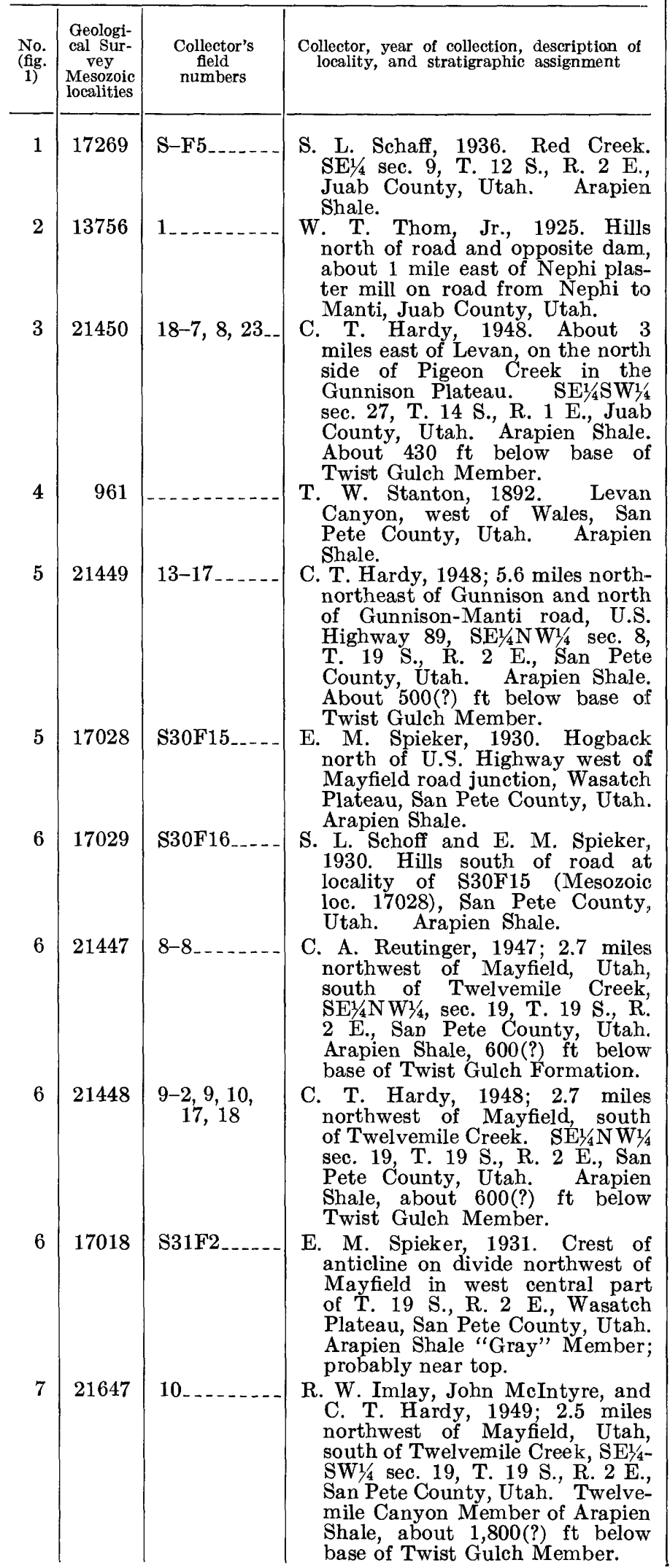

Localities at which marine megafossils of Jurassic age have been collected in central and southern Utah-Continued

\begin{tabular}{|c|c|c|c|}
\hline $\begin{array}{l}\text { No. } \\
\text { (fig. } \\
\text { 1) }\end{array}$ & $\begin{array}{l}\text { Geologi- } \\
\text { cal Sur- } \\
\text { vey } \\
\text { Mesozoic } \\
\text { localities }\end{array}$ & $\begin{array}{l}\text { Collector's } \\
\text { feld } \\
\text { numbers }\end{array}$ & $\begin{array}{l}\text { Collector, year of collection, description of } \\
\text { locality, and stratigraphic assignment }\end{array}$ \\
\hline 8 & 21446 & $7-21,25,30_{-}$ & $\begin{array}{l}\text { C. T. Hardy, 1948; } 1.9 \text { miles north- } \\
\text { west of Mayfield, NE⿺辶 NW1/4 sec. } \\
\text { 30, T. 19 S., R. 2 E., San Pete } \\
\text { County, Utah. Arapien Shale, } \\
\text { about 1,800(?) ft below base of } \\
\text { Twist Gulch Member. } \\
\text { E. M. Spieker, 1930. Near May- } \\
\text { field-Gunnison road about } 1 \text { mile } \\
\text { from Mayfield, Wasatch Plateau, } \\
\text { San Pete County, Utah. Arapien } \\
\text { Shale. }\end{array}$ \\
\hline
\end{tabular}
Shale.

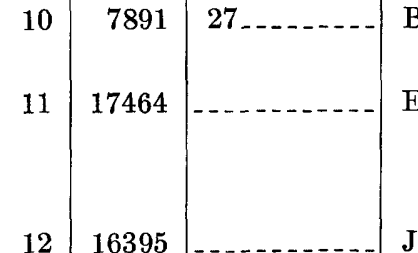

12

16395

17462

45

S. Butler, 1912. Cottonwood Canyon, Marysvale district, Piute County, Utah. Float, upper limestone.

B. S. Butler, 1912. Cottonwood Canyon, Marysvale District, Piute County, Utah.

Eugene Callaghan, 1937. Tushar Mountains, near the north side of sec. 24 , T. 28 S., R. 4 W., Piute County, Utah. From a gray well-bedded limestone outcrop.

J. W. Young, 1933 . Limestone Gulch, 8 miles south of Marysvale, sec. 20 , T. 28 S., R. 4 W., Piute County, Utah. Arapien Shale.

Eugene Callaghan, 1937. From Limestone Gulch in sec. 30, T. 28 S., R. 3 W., Piute County, Utah. Approximately at edge of Delano Peak No. 2 quadrangle.

17463 ugene Callaghan, 1937. From exposures near the road on south side of Cottonwood Creek canyon in sec. 26 [probably error for sec. 23], T. 28 S., R. 4 W., Tushar Mountains, Piute County, Ut ah

26310 W. Imlay, 1956. About 3-miles up Cottonwood Creek, southwest of Marysvale, sec. 20, T. 28 S., R. 4 W., Piute County, Utah. Limestone float on road, probably derived from just above Navajo Sandstone.

P. E. Dennis, 1941. Near Minersville, Beaver County, Utah.

P. E. Dennis, 1941 . Near Minersville, Beaver County, Utah.

P. E. Dennis, 1941. Northeast of Minersville, Beaver County, Utah.

18672

220.

18211

H. E. Thomas, 1940. Iron Springs quadrangle, $\mathrm{SE}^{1 / 4} \mathrm{NW}^{1 / 4}$ sec. 21 , T. 35 S., R. 12 W., Iron County, Utah. Carmel Formation. Dense blue limestone mapped by Leith and Harder as the Homestake limestone.
17

19423

17
G. C. Selfridge, 1943. From east side of Iron Mountain in the Iron Springs area. T. 35 S., R. 12 W., Iron County, Utah. Carmel Formation.

25674 1955. From one-half mile east of Iron Springs on paved highway to Cedar City, Iron County, Utah. Carmel Formation. 
Localities at which marine megafossils of Jurassic age have been collected in central and southern Utah-Continued

\begin{tabular}{|c|c|c|c|}
\hline $\begin{array}{l}\text { No. } \\
\text { (fig. } \\
\text { 1) }\end{array}$ & $\begin{array}{l}\text { Geologi- } \\
\text { cal Sur- } \\
\text { vey } \\
\text { Mesozoic } \\
\text { localities }\end{array}$ & $\begin{array}{c}\text { Collector's } \\
\text { field } \\
\text { numbers }\end{array}$ & $\begin{array}{l}\text { Collector, year of collection, description of } \\
\text { locality, and stratigraphic assignment }\end{array}$ \\
\hline 18 & 18213 & C-36-11_. & $\begin{array}{l}\text { H. E. Thomas, 1940; } 2 \text { miles north } \\
\text { of Cedar City, NE1/4 NE1/4, sec. 2, } \\
\text { T. } 36 \text { S., R. 11 W., Iron County, } \\
\text { Utah. Buff to brown limestone } \\
\text { of the Carmel Formation. Posi- } \\
\text { tion uncertain. }\end{array}$ \\
\hline 19 & 19428 & 121-bed 23.- & $\begin{array}{l}\text { H. J. Bissell, 1944. Sec. 1, T. } 36 \\
\text { S., R. 11 W., northeast of Cedar } \\
\text { City, Iron County, Utah. Car- } \\
\text { mel Formation. Main Penta- } \\
\text { crinus unit. From lower part of } \\
23 \mathrm{ft} \text { of fossiliferous limestone } \\
\text { lying } 231 \mathrm{ft} \text { above base of Carmel. }\end{array}$ \\
\hline 19 & 19429 & $122-\mathrm{A}$ & $\begin{array}{l}\text { H. J. Bissell, 1944. Sec. 1, T. } 36 \\
\text { S., R. } 11 \text { W., northeast of Cedar } \\
\text { City, Iron County, Utah. Car- } \\
\text { mel Formation, } 203 \mathrm{ft} \text { above base. }\end{array}$ \\
\hline 19 & 19431 & $123 \ldots$ & $\begin{array}{l}\text { H. J. Bissell, 1944, Sec. 1, T. } 36 \\
\text { S., R. 11 W., northeast of Cedar } \\
\text { City, Iron County, Utah. Car- } \\
\text { mel Formation, from } 331 \text { to } 369 \\
\text { ft above base. }\end{array}$ \\
\hline 19 & 19432 & $122 \ldots$ & $\begin{array}{l}\text { H. J. Bissell, 1944. Sec. 1, T. } 36 \\
\text { S., R. 11 W., northeast of Cedar } \\
\text { City, Iron County, Utah. Car- } \\
\text { mel Formation. Bed } 24 \text { of Bis- } \\
\text { sell's measured section. Main } \\
\text { Nucula-bearing unit, 11 ft of } \\
\text { limonitic to yellow-brown lime- } \\
\text { stone and siltstone lying about } \\
254 \mathrm{ft} \text { above base of formation. }\end{array}$ \\
\hline 20 & 18660 & 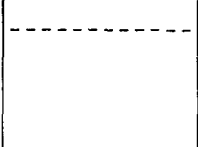 & $\begin{array}{l}\text { H. E. Gregory, 1941. Coal Creek } \\
\text { Canyon, sec. 15(?) T. } 35 \text { S., R. } \\
101 / 2 \text { W. East of Cedar City, } \\
\text { Iron County, Utah. Carmel } \\
\text { Formation. }\end{array}$ \\
\hline 21 & 25672 & I-55-7-19A_ & $\begin{array}{l}\text { R. W. Imlay, Paul Averitt, and } \\
\text { Hector Ugalde, 1955. North } \\
\text { side of Shurtz Creek about } 6 \\
\text { miles south of Cedar City, secs. } \\
11 \text { and } 14 \text {, T. } 37 \text { S., R. 11 W., } 11 \text {. } \\
\text { Iron County, Utah. Carmel } \\
\text { Formation. From pebbly oolitic } \\
\text { limestone in lower part of for- } \\
\text { mation about } 235 \mathrm{ft} \text { above base. }\end{array}$ \\
\hline 21 & 26309 & & $\begin{array}{l}\text { R. W. Imlay, } 1956 . \text { Along Shurtz } \\
\text { Creek Road in sec. 11, T. } 37 \text { S., } \\
\text { R. } 11 \text { W., Iron County, Utah. } \\
\text { Carmel Formation, oolitic sandy } \\
\text { limestone overlying cliff-forming } \\
\text { limestone whose top is } 235 \mathrm{ft} \\
\text { above top of Navajo Sandstone. }\end{array}$ \\
\hline 21 & 27470 & SS-II-A & $\begin{array}{l}\text { J. C. Wright and R. P. Snyder, } 1959 . \\
\text { On crest line of north pyramid } \\
\text { near Shurtz Creek. SW1/4W } 114 \\
\text { sec. 11, T. } 37 \text { S., R. 11 W., Iron } \\
\text { County, Utah. Carmel Forma- } \\
\text { tion, } 458 \text { ft above base. }\end{array}$ \\
\hline 21 & 27471 & S-I-E & $\begin{array}{l}\text { J. C. Wright and R. P. Snyder, } \\
1959 \text {. 314 ft above base of } \\
\text { Carmel Formation. Same local- } \\
\text { ity as } 27470 \text {. }\end{array}$ \\
\hline 21 & 27472 & S-I-D_- & $\begin{array}{l}\text { J. C. Wright and R. P. Snyder, } \\
\text { 1959. Shurtz Creek, 307-310 ft } \\
\text { above base of Carmel Formation. } \\
\text { Same locality as } 27470 \text {. }\end{array}$ \\
\hline 21 & 27473 & S-I-C & $\begin{array}{l}\text { J. C. Wright and R. P. Snyder, } \\
\text { 1959. Shurtz Creek, } 287 \mathrm{ft} \text { above } \\
\text { base of Carmel Formation. Same } \\
\text { locality as } 27470 \text {. }\end{array}$ \\
\hline
\end{tabular}

Localities at which marine megafossils of Jurassic age have been collected in central and southern Utah-Continued

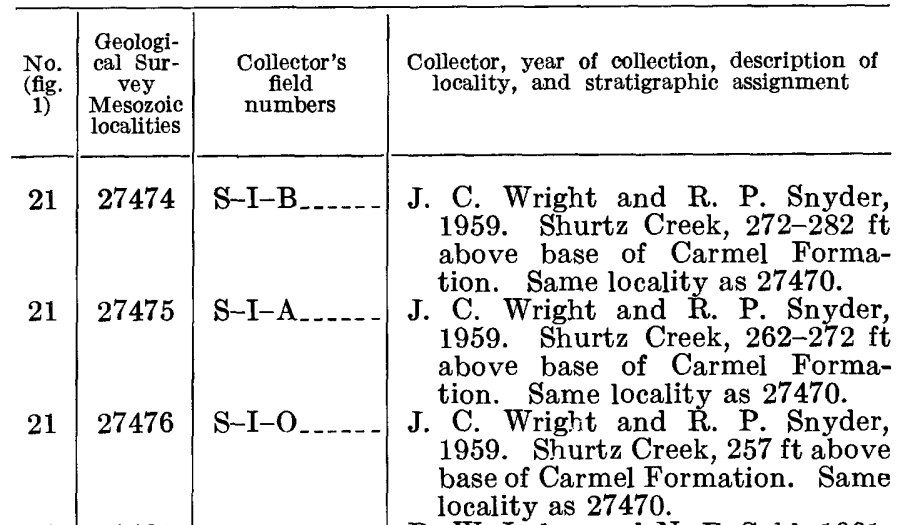

R. W. Imlay and N. F. Sohl, 1961. North side of Shurtz Creek about 6 miles south of Cedar City. Secs. 11 and 14, T. 37 S., R. 11 W., Iron County, Utah. Carmel Formation.

R. W. Imlay and N. F. Sohl, 1961. Iron County, Utah, 229-255 ft above base of Carmel Formation. Same locality as collection loc. 28461 .

G. C. Selfridge, 1943 , Just east of contact of porphyry and sedimentary deposits on Pilot Creek side of spur at Pilot Creek and Moody Wash, sec. 31, T. 36 S., R. 13 W., Iron County, Utah. Carmel Formation. Slabby gray oolitic limestone.

G. C. Selfridge, 1943 . Pink oolitic limestone from the Moody Wash side of the spur at same locality as loc. 19424.

G. C. Selfridge, 1943. Granite Mountain, just east of the Armstrong ore body. SW14 sec. 32 , T. 35 S., R 12 W., Iron County, Utah. Carmel Formation. Dense dark-gray impure limestone.

G.B.R. $5 \ldots$

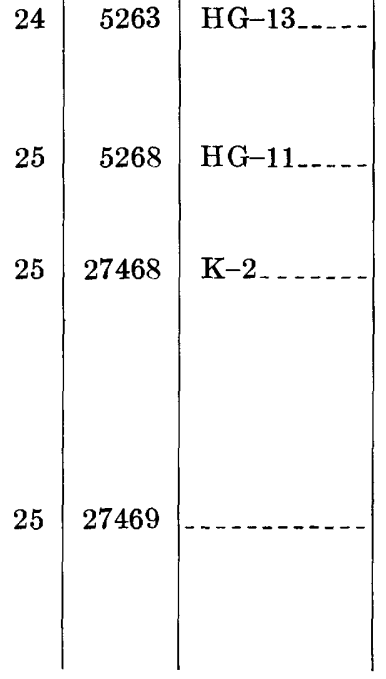

G. B. Richardson, 1907. Road from Kanarraville to coal mine, Utah. Near NW114 cor. sec. 29, T. 37 S., R. 11 W., Iron County, Utah. Altitude 7,000 ft. Carmel Formation.

G. B. Richardson and H. Graff, 1907. W1/2 sec. 32 , T. 37 S., R. 11 W., Iron Co., Utah. Carmel Formation, 1 mile southwest of Union coal mine, Kanarraville.

H. Graff, 1907 ; 1 mile east of Kanarraville, sec. 1, T. 38 S., R. 12 W., Iron County, Utah. Carmel Formation.

J. C. Wright and R. P. Snyder 1959. NE $1 / 4$ sec. $7, T$, 38 S., $R$. $11 \mathrm{~W}$. On southwest spur of knoll southwest of Sweetwater Spring about 4 miles southeast of Kanarraville. Gray dense limestone from 555 to $575 \mathrm{ft}$ above base of Carmel Formation, Iron County, Utah.

J. C. Wright and R. P. Snyder, 1959; 4 miles southeast of Kanarraville, $\mathrm{NE}^{1 / 4}$ sec. 7 , T. $38 \mathrm{~S}$., $\mathrm{R}$. 11 W., Iron County, Utah; 260$305 \mathrm{ft}$ above base of Carmel Formation. 
Localities at which marine megafossils of Jurassic age have been collected in central and southern Utah-Continued

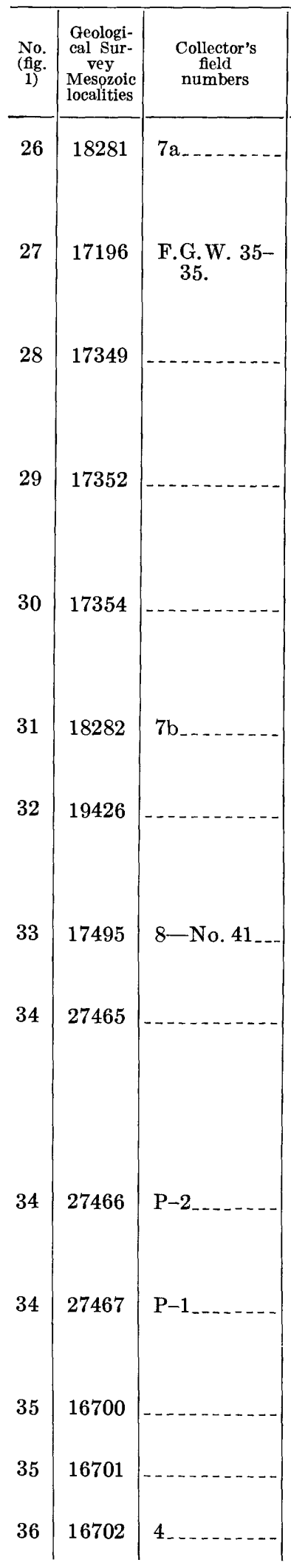

Collector, year of collection, description of locality, and stratigraphic assignment

H. E. Gregory, 1940. Horse Ranch Mountain, near E. $1 / 4$ cor. sec. 23, T. 38 S., R. 12 W. Washington County, Utah, Carmel Formation.

F. G. Wells, 1935. Limestone on hogback in $\mathrm{SW}^{1} / 4 \mathrm{SW}^{1} / 4$ sec. 26 , T. 38 S., R. 12 W., Washington County, Utah, Carmel Formation.

H. E. Gregory, 1936. Jolly Gulch, Zion Park. Head of La Verkin Creek, Washington County, Utah. Carmel Formation, topmost bed, $100 \mathrm{ft}$ below "Dakota" Sandstone.

H. E. Gregory, 1936. Horse Passture flats, $1 \frac{1}{2}$ miles northwest of road near Blue Springs, sec. 10, T. 39 S., R. 11 W., Washington County, Utah. Near top of Carmel Formation.

H. E. Gregory, 1936; 21/2 miles south of Blue Springs near limestone sinks, overlaid by lava. Sec. 15 , T. 39 S., R. 11 W., Washington County, Utah. Carmel Formation about $200 \mathrm{ft}$ above base.

H. E. Gregory, 1940 . Deep Creek near junction with North Fork, Virgin River, Washington County, Utah. Carmel Formation.

G. C. Selfridge, 1943. First main canyon to the east of Echo Spring Canyon, sec. 35, T. 40 S., R. 10 W., Washington County, Utah. Carmel Formation, pinkish-gray oolitic limestone.

H. E. Gregory, 1937. West of Pintura, sec. 32, T. 39 S., R. 13 W., Washington County, Utah. Carmel Formation

J. C. Wright and R. P. Snyder 1959. Just north of Mill Creek at Red Seep, 3.3. miles along road from east boundary of National Forest, sec. 29, T. 39 S., R. 13 W., Washington County, Utah. Carmel Formation. Yellowish silty limestone about $10 \mathrm{ft}$ below top.

J. C. Wright and R. P. Synder 1959. Same as USGS loc. 27465 . Washington County, Utah. Carmel Formation, about $75 \mathrm{ft}$ below top. 1959. Same location as USGS loc. 27465. Washington County Utah. Carmel Formation, about 250-350 ft above base.

C. E. Dobbin, 1934 . Sec. 22, T. 41 S., R. 13 W., Washington County, Utah. Carmel Formation.

C. E. Dobbin, 1953 . Sec. 22, T. 41 S., R. 13 W., Washington County, Utah. Carmel Formation.

C. E. Dobbin, 1934. NE $1 / 4$ sec. 34 T. 40 S., R. 14 W., Washingtor
J. C. Wright and R. P. Snyder
Localities at which marine megafossils of Jurassic age have been collected in central and southern Utah-Continued

\begin{tabular}{|c|c|c|c|}
\hline $\begin{array}{l}\text { No. } \\
\text { (fig. } \\
\text { 1) }\end{array}$ & $\begin{array}{l}\text { Geologi- } \\
\text { cal Sur- } \\
\text { vey } \\
\text { Mesoonoic } \\
\text { localities }\end{array}$ & $\begin{array}{l}\text { Collector's } \\
\text { feld } \\
\text { numbers }\end{array}$ & $\begin{array}{l}\text { Collector, year of collection, description of } \\
\text { locality, and stratigraphic assignment }\end{array}$ \\
\hline 36 & 16703 & $5 \ldots$ & $\begin{array}{l}\text { County, Utah. Carmel Forma- } \\
\text { tion. } \\
\text { C. E. Dobbin, } 1934 \text {. NE1/4 sec. } 34 \text {, } \\
\text { T. } 40 \mathrm{~S} ., \mathrm{R} .14 \text { W., Washington } \\
\text { County, Utah. Carmel Forma- } \\
\text { tion. }\end{array}$ \\
\hline
\end{tabular}

R. W. Imlay and N. F. Sohl, 1961. Danish Ranch section, NE. cor. sec. 34 , T. 40 S., R. 14 W., Washington County, Utah. Carmel Formation, $60 \mathrm{ft}$ above base of limestone.

37

28475 Imlay and N. F. Sohl, 1961. Cottonwood Canyon, sec. 3, T. 41 S., R. 15 W., Washington County, Utah. Carmel Formation. Oolitic and crinoidal limestone float, probably from $170 \mathrm{ft}$ above base of limestone member, but possibly from a unit $240 \mathrm{ft}$ above base.
37

28477

39
. W. Imlay and N. F. Sohl, 1961. Sec. 3, T. 41 S., R. 15 W., Cottonwood Canyon, Washington County, Utah. From oolitic limestone zone about $140 \mathrm{ft}$ above base of limestone member.

F. G. Wells, $1935 . \quad \mathrm{NE}^{1 / 4} \mathrm{NW}^{1 / 4}$ sec. 25 , T. 38 S., R. 17 W., Washington County, Utah. Carmel Formation.

F. G. Wells, 1935 . Limestone on ridge on south side of sec. $27, \mathrm{~T}$. 38 S., R. 17 W., Washington County, Utah. Carmel Formation.

H. E. Gregory, 1931. "Mount Jackson" near Nevada line west of Gunlock on the Santa Clara River, about $60 \mathrm{ft}$ below "Dakota" Sandstone. Washington County, Utah. Carmel Formation.

Robert Anderson, 1910. Santa Clara River $1^{1 / 4}$ miles below Gunlock on northeast side of creek and northeast of Gunlock field. Washington County, Utah. Carmel Formation.

H. E. Gregory, 1931; 2 miles south of Gunlock, Santa Clara River. Washington County, Utah. Carmel Formation. About middle of $300 \pm \mathrm{ft}$ of thin-bedded limestone.

R. W. Imlay and N. F. Sohl, 1961. West side of Santa Clara River, $1 \frac{1}{2}$ miles south of Gunlock near center of sec. $32, \mathrm{~T} .40 \mathrm{~S}$., R. 17 W., Washington County, Utah. 'Carmel Formation, 287 ft above base.

R. W. Imlay and N. F. Sohl, 1961; $11 / 2$ miles south of Gunlock, west side of Santa Clara River, Washington County, Utah. Carmel Formation, $50 \mathrm{ft}$ below base of Upper Cretaceous basal conglomerate. 
Localities at which marine megafossils of Jurassic age have been collected in central and southern Utah-Continued

\begin{tabular}{|c|c|c|c|}
\hline $\begin{array}{l}\text { No. } \\
\text { (fig. } \\
\text { 1) }\end{array}$ & $\begin{array}{l}\text { Geologi- } \\
\text { cal Sur- } \\
\text { vey } \\
\text { Mesozoic } \\
\text { localities }\end{array}$ & $\begin{array}{l}\text { Collector's } \\
\text { feld } \\
\text { numbers }\end{array}$ & $\begin{array}{l}\text { Collector, year of collection, description of } \\
\text { locality, and stratigraphic assignment }\end{array}$ \\
\hline 42 & 28465 & & $\begin{array}{l}\text { R. W. Imlay and N. F. Sohl, } 1961 \text {; } \\
11 / 2 \text { miles south of Gunlock on } \\
\text { west side of Santa Clara River; } \\
\text { near center sec. 32, T. 40 S., } \\
\mathrm{R} .17 \text { W., Washington County, } \\
\text { Utah. Carmel Formation, float } \\
\text { from oolitic sandstone capping } \\
\text { ridge about } 350 \mathrm{ft} \text { above base } \\
\text { of limestone member of Carmel } \\
\text { Formation. }\end{array}$ \\
\hline 42 & 28466 & & $\begin{array}{l}\text { R. W. Imlay and N. F. Sohl, } 1961 \text {; } \\
11 / 2 \text { miles south of Gunlock, } \\
\text { Washington County, Utah. } \\
\text { Carmel Formation about } 5 \mathrm{ft} \\
\text { above loc. } 28464 \text {. }\end{array}$ \\
\hline 42 & 28467 & - & $\begin{array}{l}\text { R. W. Imlay and N. F. Sohl, 1961; } \\
1 \text { miles south of Gunlock, } \\
\text { Washington County, Utah; } 430 \\
\text { ft above base of limestone mem- } \\
\text { ber of Carmel Formation. }\end{array}$ \\
\hline 43 & 25826 & JCW10 & $\begin{array}{l}\text { J. C. Wright and D. D. Dickey, } \\
1955 \text {. At Cedar Mountain, San } \\
\text { Rafael Swell, sec. 10, T. 19 S., } \\
\text { R. } 11 \text { E., Emery County, Utah. } \\
\text { Carmel Formation, } 30 \mathrm{ft} \text { above } \\
\text { base. }\end{array}$ \\
\hline 43 & 25827 & JCW13. & $\begin{array}{l}\text { J. C. Wright and D. D. Dickey, } \\
\text { 1955. At Cedar Mountain, San } \\
\text { Rafael Swell, sec. 10, T. 19 S., } \\
\text { R. 11 E., Emery County, Utah. } \\
\text { Carmel Formation, } 30 \text { ft above } \\
\text { base. }\end{array}$ \\
\hline 44 & 28470 & $\ldots \ldots$ & $\begin{array}{l}\text { R. W. Imlay and N. F. Sohl, } 1961 . \\
\text { Tributary on northeast side of } \\
\text { Buckhorn Wash at crossing of } \\
\text { road from Woodside to Castle } \\
\text { Dale, Utah. Sec. 8, T. 19 S., } \\
\text { R. 11 E., Emery County, Utah. } \\
\text { Carmel Formation. }\end{array}$ \\
\hline 45 & 10098 & & $\begin{array}{l}\text { W. M. Small, 1917. San Rafael } \\
\text { Swell at point where Buckhorn } \\
\text { Wash goes down from the lower } \\
\text { Buckhorn Flat, SE1/4 sec. 13, } \\
\text { T.19 S., R. } 10 \text { E., Emery County, } \\
\text { Utah. Carmel Formation. }\end{array}$ \\
\hline 45 & 20351 & I-46-9-11B- & $\begin{array}{l}\text { J. B. Reeside, Jr., and R. W. } \\
\text { Imlay, 1946. Head of Buckhorn } \\
\text { Wash, sec. 13, T. } 19 \mathrm{~S} ., \mathrm{R} .10 \mathrm{E} . \text {, } \\
\text { Emery County, Utah. Carmel } \\
\text { Formation, platy limestone about } \\
25 \mathrm{ft} \text { above base. }\end{array}$ \\
\hline 45 & 20359 & $1-46-9-11 \mathrm{~B}$ & $\begin{array}{l}\text { J. B. Reeside, Jr., and R. W. Imlay, } \\
1946 \text {. Head of Buckhorn Wash, } \\
\text { sec. 13, T. } 19 \text { S., R. 10 E., } \\
\text { Emery County, Utah, Carmel } \\
\text { Formation, platy limestone about } \\
25 \mathrm{ft} \text { above base. }\end{array}$ \\
\hline 45 & 20364 & $\mathrm{I}-46-9-11 \mathrm{~A}$ & $\begin{array}{l}\text { J. B. Reeside, Jr., and R. W. Imlay, } \\
1946 . \text { Head of Buckhorn Wash. } \\
\text { T. } 19 \text { S., R. } 10 \text { E., Carmel } \\
\text { Formation, from red beds about } \\
10 \mathrm{ft} \text { above the base. }\end{array}$ \\
\hline 45 & 25684 & I-55-6-25 - & $\begin{array}{l}\text { R. W. Imlay, } 1955 . \text { SW1/4 sec. 18, } \\
\text { T. 19 S., R. } 11 \text { E., to SE1/4 sec. } \\
13 \text {, T. 19 S., R. 10 E., Emery } \\
\text { County, Utah. Carmel Forma- } \\
\text { tion, 20-40 ft above base. }\end{array}$ \\
\hline 46 & 25670 & I-55-6-28A & $\begin{array}{l}\text { R. W. Imlay, } 1955 \text {. On small } \\
\text { ridge just south of road in } \\
\text { NE } 1 / 4 \mathrm{NW}^{1 / 4} \text { sec. } 17 \text {, T. } 19 \mathrm{~S} \text {, } \\
\text { R. 11 E., Emery County Utah. }\end{array}$ \\
\hline
\end{tabular}

Localities at which marine megafossils of Jurassic age have been collected in central and southern Utah-Continued

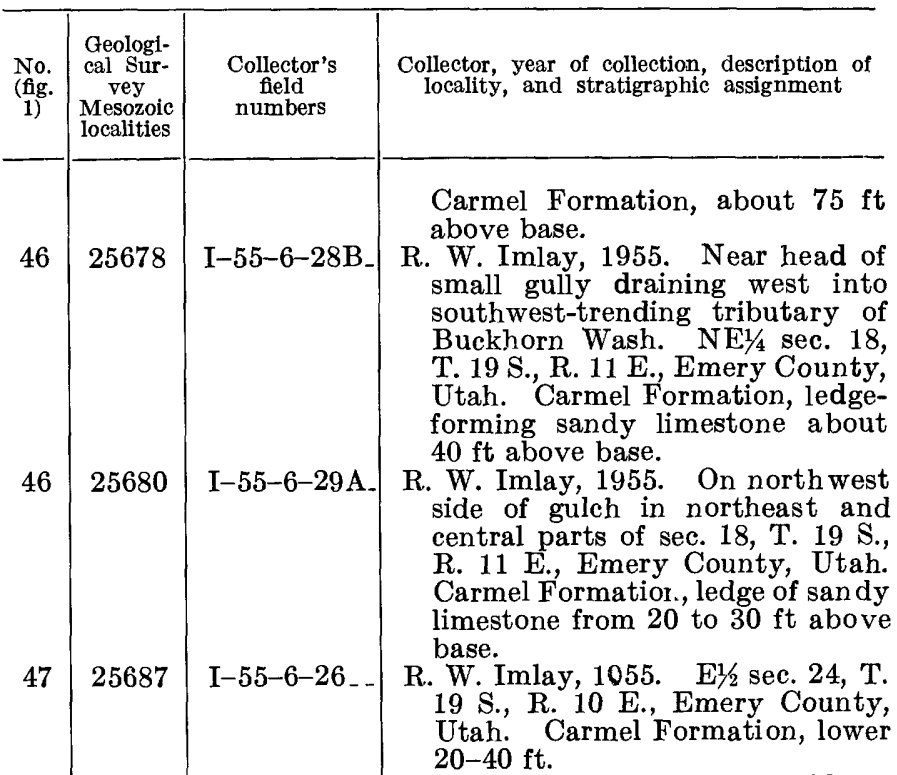

I-55-6-29B.

R. W. Imlay, 1955 . West side of gulch in $\mathrm{SW}^{1 / 4} \mathrm{SE}^{1 / 4}$ sec. $36, \mathrm{~T} .19$ S., R. 10 E., Emery County, Utah. Carmel Formation, from 15 to $25 \mathrm{ft}$ above base in sandy limestone.

48

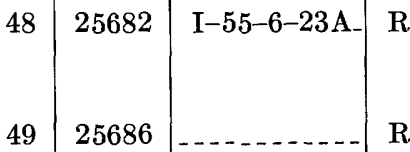

49

12555

12556

121

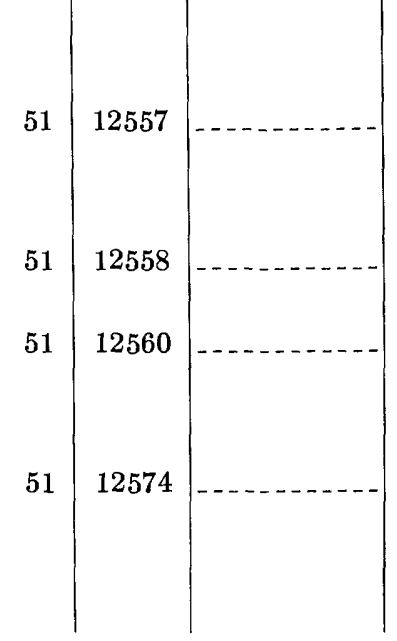

R. Imlay, 1955 NW1/4 sec. 1 , T. 20 S., R. 10 E., Emery County, Utah. Carmel Formation, about $40 \mathrm{ft}$ above base.

R. W. Imlay, $1955 . \quad \mathrm{NW}^{1 / 4}$ sec. 1 , T. 20 S., R. 10 E., Emery County, Utah. Carmel Formation, lower $20 \mathrm{ft}$.

R. W. Imlay, 1955. Northeast part of sec. 31 , T. 19 S., R. 11 E. Emery County, Utah. Carmel Formation, about $20 \mathrm{ft}$ above base.

W. H. Newhouse, 1924. The Wedge, about 2 miles south of Fullers Bottom along San Rafael River, San Rafael Swell, Emery County, Utah. Carmel Formation, 6-12 ft above Navajo Sandstone.

D. J. Fisher, 1924. The Wedge, between Buckhorn Wash and San Rafael River, Emery County, Utah. Carmel Formation, 8-10 ft above base.

W. H. Newhouse, 1924. San Rafael River below Fullers Bottom, San Rafael Swell, Emery County, Utah. Carmel Formation, $18 \mathrm{ft}$ above Navajo Sandstone.

W. H. Newhouse, 1924. San Rafael River, San Rafael Swell, Carmel Formation, $15 \mathrm{ft}$ above base

W. H. Newhouse, 1924. Below Fullers Bottom on San Rafael River, Emery County, Utah. Carmel Formation, $17 \mathrm{ft}$ above the Navajo Sandstone.

W. H. Newhouse, 1924. Below Fullers Bottom on San Rafael River. San Rafael Swell, Emery County, Utah. Carmel Formation, 2-8 ft above Navajo Sandstone. 
Localilies at which marine megafossils of Jurassic age have been collected in central and southern Utah-Continued

\begin{tabular}{|c|c|c|c|}
\hline $\begin{array}{l}\text { No. } \\
\text { (fig. } \\
1 \text { ) }\end{array}$ & $\begin{array}{l}\text { Geologi- } \\
\text { cal Sur- } \\
\text { vey } \\
\text { Mesozoic } \\
\text { localities }\end{array}$ & $\begin{array}{l}\text { Collector's } \\
\text { field } \\
\text { numbers }\end{array}$ & $\begin{array}{l}\text { Collector, year of collection, description of } \\
\text { locality, and stratigraphic assignment }\end{array}$ \\
\hline 52 & 12841 & No. L & $\begin{array}{l}\text { James Gilluly, 1924. Salt Wash } \\
\text { Canyon, sec. 25, T. 20 S., R. } 9 \text { E., } \\
\text { San Rafael Swell, Emery Count, } \\
\text { Utah. Carmel Formation, 16 } \\
\text { ft above Navajo Sandstone. }\end{array}$ \\
\hline 52 & 12842 & $M_{\ldots} \ldots$ & $\begin{array}{l}\text { James Gilluly, 1924. Salt Wash } \\
\text { Canyon, sec. 25, T. } 20 \text { S., R. } 9 \text { E., } \\
\text { San Rafael Swell, Emery County, } \\
\text { Utah. Carmel Formation, } 21 \mathrm{ft} \\
\text { above Navajo Sandstone. }\end{array}$ \\
\hline 53 & 6941 & & $\begin{array}{l}\text { Robert Forrester, } 1910 . \quad \text { Near } \\
\text { mouth of Salt Wash, Emery } \\
\text { County, Utah. Carmel Forma- } \\
\text { tion. }\end{array}$ \\
\hline 54 & 6944 & & $\begin{array}{l}\text { Robert Forrester, } 1910 \text {. Coal } \\
\text { Wash, about 2 miles above } \\
\text { Leaking Springs, Emery County, } \\
\text { Utah. Carmel Formation. }\end{array}$ \\
\hline 54 & 7341 & $\mathbf{F}-33 \ldots$ & $\begin{array}{l}\text { T. Lupton, 1911. About } 2 \\
\text { miles above spring on Coal Wash } \\
\text { east of Ferron, Emery County, } \\
\text { Utah. Carmel Formation, just } \\
\text { above massive Navajo Sandstone. }\end{array}$ \\
\hline 55 & 6281 & & $\begin{array}{l}\text { Robert Forrester, } 1909 \text {. Coal } \\
\text { Wash, sec. 10, T. } 21 \mathrm{~S} . \text {, R. } 9 \text { E., } \\
\text { Emery County, Utah. Carmel } \\
\text { Formation, in lower } 30 \mathrm{ft} \text {. }\end{array}$ \\
\hline 56 & 13529 & No. ABCD_ & $\begin{array}{l}\text { James Gilluly, and E. T. McKnight, } \\
1925 \text {. Rim of Eagle Canyon, } \\
\text { just below road from Rochester } \\
\text { to Horn Silver Prospect, approxi- } \\
\text { mately sec. 33, T. } 22 \text { S., R. } 9 \text { E. } \\
\text { (unsurveyed), Emery County, } \\
\text { Utah. Basal limestone of Carmel }\end{array}$ \\
\hline 56 & 25673 & I- $-55-7-8 A_{--.}$ & $\begin{array}{l}\text { Formation. } \\
\text { R. W. Imlay, } 1955 \text {. On divide } \\
\text { between Eagle Canyon and } \\
\text { Devil's Canyon, NE1/4 sec. } 33 \text {, } \\
\text { T. } 22 \text { S., R. } 9 \text { E., Emery County, } \\
\text { Utah. Carmel Formation, about } \\
15 \mathrm{ft} \text { above base. }\end{array}$ \\
\hline 56 & 27592 & & $\begin{array}{l}\text { J. S. Wright and D. D. Dickey, } \\
1956 \text {. On high flatiron just } \\
\text { south of Eagle Canyon near } \\
\text { road on west side of San Rafael } \\
\text { Swell. NE1/4 sec. 33, T. 22 S., } \\
\text { R. } 9 \text { E., Emery County, Utah. } \\
\text { Carmel Formation, limestone } \\
\text { member. }\end{array}$ \\
\hline 56 & 28472 & & $\begin{array}{l}\text { R. W. Imlay and N. F. Sohl, } 1961 . \\
\text { Justensen Flats, NW1/4 sec. 3, } \\
\text { T. } 23 \text { S., R. } 9 \text { E., Emery County, } \\
\text { Utah. Limestone ledges between } \\
\text { switchbacks of road, 14-21 ft } \\
\text { above base of Carmel Formation. }\end{array}$ \\
\hline 56 & 28480 & & $\begin{array}{l}\text { R. W. Imlay and N. F. Sohl, } 1961 \text {. } \\
\text { Eagle Canyon Lookout, sec. 33, } \\
\text { T. } 22 \mathrm{~S} \text {., R. } 9 \text { E., Emery County, } \\
\text { Utah. About } 20 \mathrm{ft} \text { above base } \\
\text { of Carmel Formation in bed } \\
\text { of limestone with very abundant } \\
\text { fossil fragments just below green } \\
\text { shale. }\end{array}$ \\
\hline 57 & 6282 & No. $3 \mathrm{Cc}_{-}$ & $\begin{array}{l}\text { Robert Forrester, 1909. } \\
\text { Canyon, sec. 14, T. 23 } \\
8 \text { S., R. R. } \\
8 \text { E., Emery County, Uttah. } \\
\text { Carmel Formation, in lower } \\
30 \mathrm{ft} .\end{array}$ \\
\hline 58 & 13531 & B-1_.- & $\begin{array}{c}\text { James } \\
\text { Creek, }\end{array}$ \\
\hline
\end{tabular}

Localities at which marine megafossils of Jurassic age have been collected in central and southern Utah-Continued

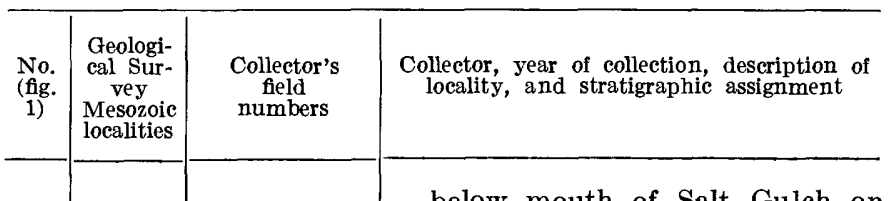
west flank of San Rafael Swell, Emery County, Utah. Carmel Formation, $17 \mathrm{ft}$ above base.

58 1

12839

No. F

No. G

No

No. $B$ 
Localities at which marine megafossils of Jurassic age have been collected in central and southern Utah-Continued

\begin{tabular}{|c|c|c|c|}
\hline $\begin{array}{l}\text { No. } \\
\text { (fig. } \\
\text { 1) }\end{array}$ & $\begin{array}{l}\text { Geologi- } \\
\text { cal Sur- } \\
\text { vey } \\
\text { Mesozoic } \\
\text { localities }\end{array}$ & $\begin{array}{l}\text { Collector's } \\
\text { field } \\
\text { numbers }\end{array}$ & $\begin{array}{l}\text { Collector, year of collection, description of } \\
\text { locality, and stratigraphic assignment }\end{array}$ \\
\hline 65 & 12581 & & $\begin{array}{l}\text { J. B. Reeside, Jr., } 1924 \text {. About } 15 \\
\text { miles southwest of Green River, } \\
11 / 2 \text { miles west of Lost Spring, San } \\
\text { Rafael Swell, Emery County, } \\
\text { Utah: Carmel Formation, } 104 \mathrm{ft} \\
\text { above top of Navajo Sandstone. }\end{array}$ \\
\hline 65 & 16202 & No. H51_. & $\begin{array}{l}\text { L. G. Henbest, } 1930 . \text { Green River } \\
\text { Desert, NE1/4 sec. 19, T. } 24 \text { S., } \\
\text { R. } 13 \text { E., Emery County, Utah. } \\
\text { Carmel Formation. }\end{array}$ \\
\hline 66 & 9111 & & $\begin{array}{l}\text { B. S. Butler, 1914. Lost Spring } \\
\text { just east of San Rafael Swell, sec. } \\
29 \text {, T. } 24 \text { S., R. } 13 \text { E., Emery } \\
\text { County, Utah. Carmel Forma- } \\
\text { tion. }\end{array}$ \\
\hline 67 & 8597 & No. $80_{-}$ & $\begin{array}{l}\text { C. T. Lupton, } 1913 . \text { Green River } \\
\text { Desert, secs. 23, 24, 26, 35 T. } \\
24 \text { S., R. 12 E., Emery County, } \\
\text { Utah. Carmel Formation. }\end{array}$ \\
\hline 67 & 12570 & & $\begin{array}{l}\text { J. B. Reeside, Jr., 1924. West of } \\
\text { Lost Spring, secs. 23, 24, 26, 35, } \\
\text { T. } 24 \text { S., R. 12 E., Emery County, } \\
\text { Utah. Carmel Formation, up- } \\
\text { permost limestone (oolite), } 100 \mathrm{ft} \\
\text { above base. }\end{array}$ \\
\hline 68 & 11137 & Lot 2 . & $\begin{array}{l}\text { C. J. Hares, 1921. East side of San } \\
\text { Rafael Swell, near Temple Moun- } \\
\text { tains, sec. } 34 \text { T., } 24 \text { S., R. } 12 \text { E., } \\
\text { Emery County, Utah. Carmel } \\
\text { Formation, limestone at bottom. }\end{array}$ \\
\hline 69 & 17412 & 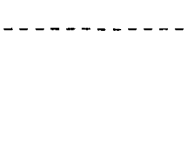 & $\begin{array}{l}\text { A. A. Baker, } 1930 \text {. About } 500 \text { feet } \\
\text { east of Swazey's Seep, Green } \\
\text { River Desert, Emery County, } \\
\text { Utah. Upper part of Carmel } \\
\text { Formation. }\end{array}$ \\
\hline 70 & 10116 & No. 11 . & $\begin{array}{l}\text { W. B. Emery, 1917. Hanksville } \\
\text { road, } 2 \text { miles south of Straight } \\
\text { Wash, Green River Desert, SE1/4 } \\
\text { sec. 6, T. } 24 \text { S., R. } 13 \text { E., Emery } \\
\text { County, Utah. Carmel Forma- } \\
\text { tion. }\end{array}$ \\
\hline 71 & 27591 & SF-1 & $\begin{array}{l}\text { J. C. Wright, } 1955 . \text { Little Wild } \\
\text { Horse Mesa, SE1/4 sec. } 35 \text {, T. } 25 \\
\text { S., R } 10 \text { E., Emery County, } \\
\text { Utah. Carmel Formation; } 42- \\
44^{1 / 2} \mathrm{ft} \text { above base. }\end{array}$ \\
\hline 72 & 24258 & CR-108_ & $\begin{array}{l}\text { J. F. Smith, } 1952 \text {. About } 1 / 4 \text { mile } \\
\text { west of Sun Glo Park (in Fish } \\
\text { Lake National Forest) Capitol } \\
\text { Reef area, SW } 1 / 4 \text { sec. 31, T. } 28 \text { S., } \\
\text { R. } 4 \text { E., Wayne County, Utah. } \\
\text { Carmel Formation, about } 40-60 \\
\text { ft above base. }\end{array}$ \\
\hline 73 & 24259 & CR-110 . & $\begin{array}{l}\text { J. F. Smith, 1952. Approximately } \\
\text { 1/2 mile west of Teasdale, Capitol } \\
\text { Reef area, sec. } 17 \text { T. } 29 \text { S., R. } 4 \\
\text { E., Wayne County, Utah. Car- }-{ }^{4} \text { - Way } \\
\text { mel Formation, 40-60 ft above } \\
\text { base. }\end{array}$ \\
\hline 73 & 24260 & CR-115. & $\begin{array}{l}\text { J. F. Smith, } 1952 . \text { Approximately } \\
\text { 1/2 mile west of Teasdale, Capitol } \\
\text { Reef area, sec. } 17, \text { T. } 29 \text { S., R. } \\
4 \text { E., Wayne County, Utah. Car- } \\
\text { mel Formation. }\end{array}$ \\
\hline 73 & 25669 & I-55-7-13 . & $\begin{array}{l}\text { R. W. Imlay, } 1955 \text {. One-half mile } \\
\text { west of Teasdale, sec. 17, T. } 29 \mathrm{~S} \text {., } \\
\text { R. } 4 \text { E., Wayne County, Utah. } \\
\text { Carmel Formation. Oolitic lime- } \\
\text { stone, about } 44 \mathrm{ft} \text { above top of } \\
\text { Navajo Sandstone, or } 7 \mathrm{ft} \text { above } \\
\text { top of red unit at base of Carmel. }\end{array}$ \\
\hline
\end{tabular}

Localities at which marine megafossils of Jurassic age have been collected in central and southern Utah-Continued

\begin{tabular}{c|c|c}
\hline $\begin{array}{c}\text { No. } \\
\text { (fig. } \\
1 \text { ) }\end{array}$ & $\begin{array}{c}\text { Geologi- } \\
\text { cal Sur- } \\
\text { vey } \\
\text { Mesozoic } \\
\text { localities }\end{array}$ & $\begin{array}{c}\text { Collector's } \\
\text { feld } \\
\text { numbers }\end{array}$
\end{tabular}
West of Teasdale, between town and Bullberry Creek, SE1/4 SE1/4 sec. 18, T. 29 S., R. 4 E., Wayne County, Utah. Carmel Formation.

7

180

18076

No. 4.....

E. Gregory, 1937. In Fremont River Canyon, 4 miles below Fruita, west-central part of sec. 17, T. 29 S., R. 7 E., Wayne County, Utah. Carmel Formation.

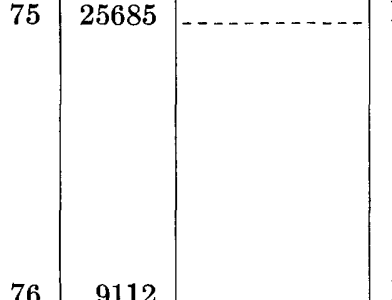

76

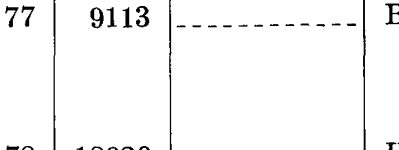

78

18920

78

79

28492

80

25671
F. Davis, 1920. East side of the Water Pocket fold just north of Capitol Wash, $\mathrm{NW}^{1 / 4}$ sec. 10 , $\mathrm{T}$. 30 S., R. 7 E., Wayne County, Utah. Carmel Formation.

R. W. Imlay, 1955. North side of mouth of Capitol Wash, at east end of Capitol Reef Monument, $\mathrm{NW}^{1 / 4}$ sec. 10 , T. 30 S., R. 7 E., Wayne County, Utah. Carmel Formation. Oolitic bed at top of limestone ledge, about $81 \mathrm{ft}$ above top of massive Navajo Sandstone and $28 \mathrm{ft}$ above gypsum bed.

B. S. Butler, 1914. On trail about midway between Hanksville and Robbers Roost just above Navajo Sandstone, $\mathrm{NE}^{1 / 4}$ sec. 31, T. 28 S., R. 13 E., Wayne County, Utah. Carmel Formation.

S. Butler, 1914. Boulder Mountain, near head of Pleasant Creek, T. 31 S., R. 5 E., Garfield County, Utah. Carmel Formation.

H. E. Gregory, 1943. Antimony Canyon, sec. 20, T. 31 S., R. 1 W., Garfield County, Utah. Carmel Formation, $10 \mathrm{ft}$ above Navajo Sandstone.

H. E. Gregory, 1943. Antimony Creek, sec. 20, T. 31 S., R. 1 W., Garfield County, Utah. Carmel Formation.

R. W. Imlay, 1956. On west side of gulch draining south into Antimony Creek in west-central part of sec. 20, T. 31 S., R. 1 W., Garfield County, Utah. Carmel Formation, $20-30 \mathrm{ft}$ above base. N. F. Sohl, 1961. Devils Backbone, approximately sec. 5 , T. 33 S., R. 3 E., Garfield County, Utah. About 200 yds south of pre-1961 Devils Backbone Bridge on west side of road. Carmel Formation, about $15-20 \mathrm{ft}$ above base.

R. W. Imlay, 1955. Deep Creek, about 1 mile southeast of road to Hell's Backbone Ridge and about 15 miles north of Escalante, Garfield County, Utah. Carmel Formation, from basal bed of $15-\mathrm{ft}$ limestone ledge about $20 \mathrm{ft}$ above top of Navajo Sandstone. 
Localities at which marine megafossils of Jurassic age have been collected in central and southern Utah-Continued

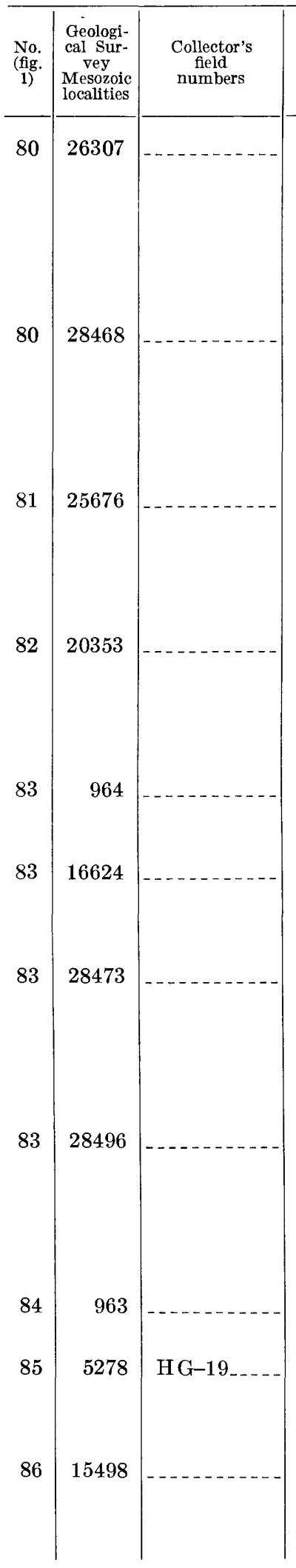

R. W. Imlay, J. D. Strobell, Jr., J. C. Wright, D. D. Dickey, 1956. On Deep Creek, onehalf mile southeast of road to Hell's Backbone, east-central part of sec. 15, T. 33 S., R. 2 E., Garfield County, Utah, Carmel Formation, shaly limestone about $12 \mathrm{ft}$ above Navajo Sandstone.

R. W. Imlay and N. F. Sohl, 1961. East side of Deep Creek 4,000 ft downstream from road crossing of Deep Creek by the road to Devil's Backbone, sec. 15, T. 33 S., R. 2 E., Garfield County, Utah. Same as loc. 26307. Carmel Formation.

R. W. Imlay, 1955. In Paria Valley, about 5 miles south of Cannonville, Kane County, Utah. Carmel Formation about $12 \mathrm{ft}$ above base of formation in gray shaly limestone. (See Gregory, 1951, p. 58.)

H. E. Gregory, 1946. Averitt Hollow, probably $\mathrm{NW}^{1 / 4}$ sec. 23 , T. 38 S., R. 3 W., Kane County, Utah. From the calcareous sandstone above the thick bed of gypsum in the "Curtis" Formation of Gregory.

T. W. Stanton, 1892 . Upper part of Kanab Creek, sec. 3, T. 41 S., R. 6 W., Kane County, Utah. Carmel Formation.

C. D. Walcott, 1879. Upper end of box canyon below volcano, Kanab Creek, $W^{1 / 2}$ sec. 3, T. 41 S., R. 6 W. Kane County, Utah. Carmel Formation.

R. W. Imlay and N. F. Sohl, 1961. Kanab Creek, west fork at Box Canyon just below Glendale Road and below lava flow, $W^{1 / 2}$ of sec. 3, T. 41 S., R. 6 W., Kane County, Utah. Carmel Formation, about 10-15 ft below top of imestone member.

R. W. Imlay and N. F. Sohl, 1961. Kanab Creek, west fork at Box Canyon just below Glendale Road and below lava flow, $W^{1 / 2}$ of sec. 3 , T. 41 S., R. 6 W., Kane County, Utah. Carmel Formation, about $10-15 \mathrm{ft}$ below top of limestone member.

T. W. Stanton, 1892. NW $\mathrm{NW}^{1 / 4}$ see. 25, T. 40 S., R. 7 W., Glendale area, Kane County, Utah.

G. B. Richardson, 1907. About $21 / 2$ miles southeast of Orderville. From limestone on top of "Sevier Fault," Kane County, Utah. Carmel Formation.

J. B. Reeside, Jr. and others, 1930. West of bridge at south end of Mount Carmel. W1/2 sec. 18, T. 41 S., R. 7 W., Kane County, Utah. Carmel Formation, $15 \mathrm{ft}$
Localities at which marine megafossils of Jurassic age have been collected in central and southern Utah-Continued

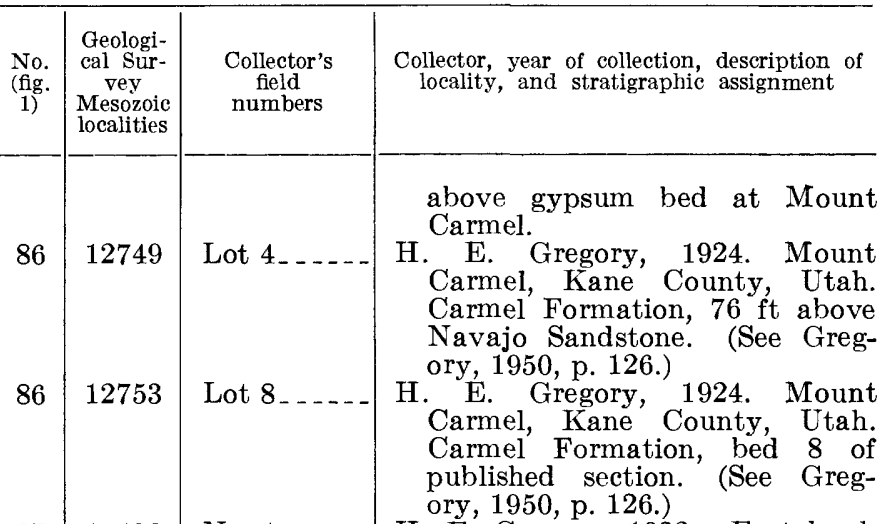

87

87

25683

87

87

28474

28469

I-55-7-21A-

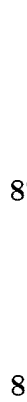

88

17353

5273 p. 126. .)

H. E. Gregory, 1922. East bank of Virgin River, about 3 miles south of Mount Carmel, sec. 30, T. 41 S., R. 7 W., Kane County, Utah. Carmel Formation.

H. E. Gregory, 1922. East bank of Virgin River, about 3 miles south of Mount Carmel, sec. 30, T. 41 S., R. 7 W., Kane County, Utah. Carmel Formation, $25 \mathrm{ft}$ above Navajo Sandstone.

R. W. Imlay and Hector Ugalde, 1955. In $\mathrm{NW}^{1 / 4}$ sec. 30 , T. $41 \mathrm{~S}$., R. 7 W., Kane County, Utah. Carmel Formation. From base of unit 10 of Gregory's published section (Gregory, 1950, p. 127).

W. Imlay and Hector Ugalde, 1955. Just southeast of bridge, in $\mathrm{SE}^{1 / 4}$ sec. 31 , T. 41 S., R. $7 \mathrm{~W}$., Kane County, Utah, Carmel Formation, top bed. Unit 15 of Gregory's published section (Gregory, 1950, p. 127).

R. W. Imlay and N. F. Sohl, 1961. Just east of highway and south of pre-1960 bridge, sec. 30, T. 41 S., R. 7 W., Kane County, Utah. Carmel Formation, about $20 \mathrm{ft}$ below top of limestone member. R. W. Imlay and N. F. Sohl, 1961. On east side of U.S. Highway 89 just south of pre-1960 bridge, sec. 30, T. 41 S., R. 7 W., Kane County, Utah. Carmel Formation, about $93 \mathrm{ft}$ above base of limestone member (at top of "Lower lime")

R. W. Imlay and N. F. Sohl, 1961. On east side of U.S. Highway 89 just south of pre-1960 bridge, sec. 30, T. 41 S., R. 7 W., Kane County, Utah. Carmel Formation, about $35 \mathrm{ft}$ below top of limestone member.

H. E. Gregory, 1936. Escarpment south of Zion-Mount Carmel road. Approximately sec. 18, T. 41 S., R. 8 W., Kane County, County, Utah. Carmel Formation.

G. B. Richardson, 1907. Valley of North Fork of Virgin River about $1 \frac{1}{2}$ miles west of Walkers Ranch, sec. 33, T. 39 S., R. 9 W., Kane County, Utah. Carmel Formation. 
Localities at which marine megafossils of Jurassic age have been collected in central and southern Utah-Continued

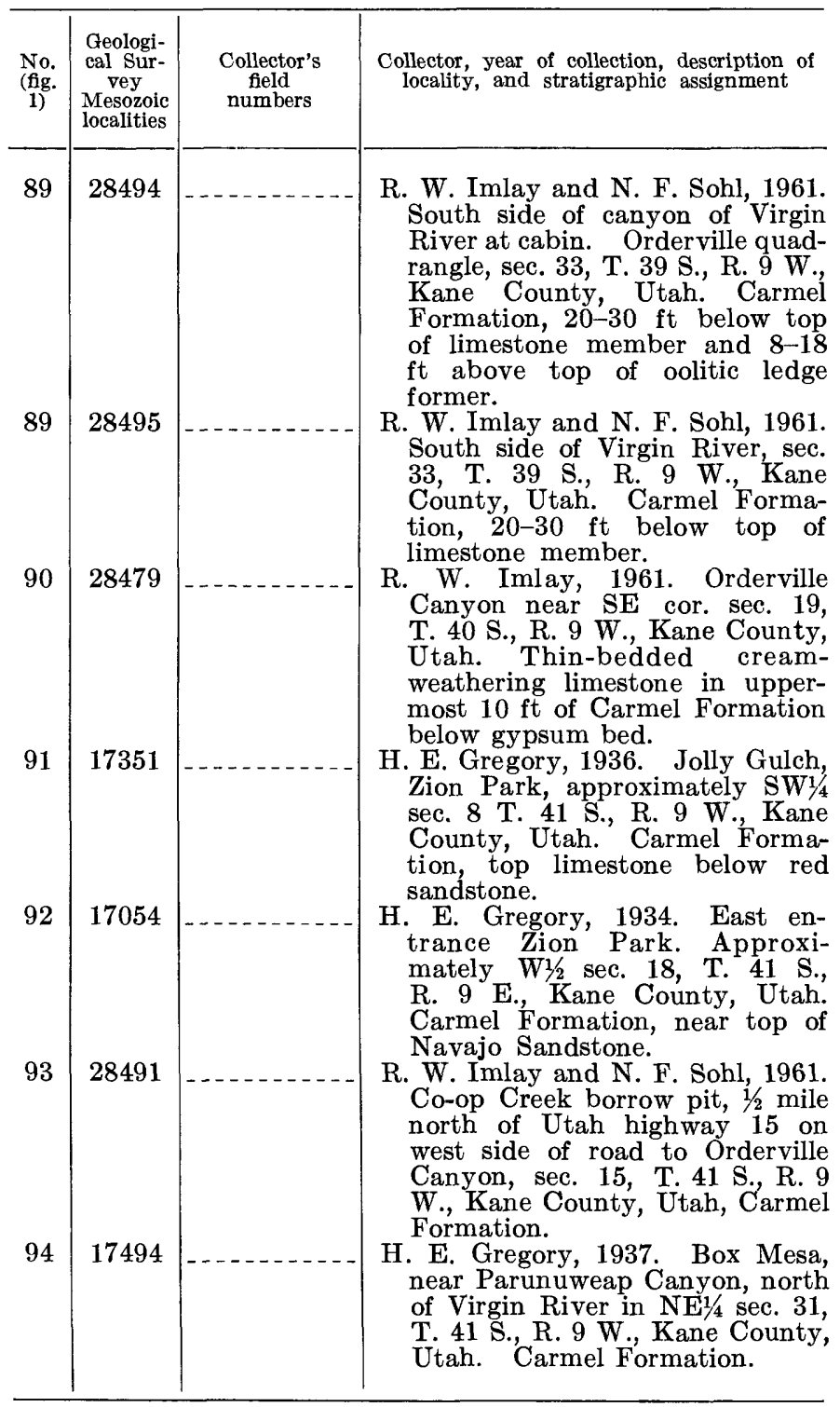

\section{SUMMARY OF RESULTS}

1. The marine Jurassic pelecypods from central and southern Utah available for study comprise about 1,700 specimens, are more common than other megafossils, and are divisible into 31 genera and 44 species. The dominant genera in order of decreasing abundance are Camptonectes, Ostrea, Pronoella, Vaugonia, Myophorella (Promyophorella), and Lima (Plagiostoma). Numerically these compose 80 percent of the specimens available for study. Nucla and Thracia are locally common but are not widespread. Grammatodon?, Modiolus, Isognomon, Plicatula, Lopha, Trigonia, Astarte (Coelastarte), and Pholadomya are fairly wide- spread but are not common. Seventeen genera occur rarely.

2. The limy lower unit of the Carmel Formation is of Bajocian to possible early Callovian age. The lower part of this unit contains pelecypods and some ammonites that are found elsewhere in the western interior region only in beds of Bajocian age. A Bajocian age for the upper part of the unit locally is suggested by the presence of the pelecypod Thracia weedi Stanton. The presence of corals and nerineid gastropods likewise favors a Bajocian age for the lower limy unit, as those organisms are uncommon above beds of that age elsewhere in the western interior.

3. This age assignment for the limy lower unit of the Carmel Formation is also favored by stratigraphic resemblances with the members of the Twin Creek Limestone of northern Utah.

4. The age of the gypsiferous claystone and siltstone composing the upper unit of the Carmel Formation is not as well established because it has furnished few fossils. It is considered to be of early Callovian age on the basis of regional stratigraphic relations.

5. The fossils found in the Twelvemile Canyon Member of the Arapien Shale are identical with those in the Twin Creek Limestone and in the limy lower unit of the Carmel Formation. One of the species present in the Twelvemile Canyon Member indicates a Bajocian age, but most of the species have a longer range elsewhere in the western interior region. Stratigraphically the Twelvemile Canyon Member is a southward extension of the Twin Creek Limestone, is overlain and underlain by nearly identical-appearing beds, and differs mainly by being more shaly and more contorted.

6. Marine Jurassic megafossils in central and southern Utah occur in greatest abundance and variety in a broad belt extending southwestward from the west side of the San Rafael Swell and become less common southeastward and northwestward from that belt. This condition is related to a gradual northwestward thickening of the associated limy beds from 0 in southeastern Utah to at least 3,000 feet in San Pete and Juab Counties. It reflects a change from slow littoral deposition on the southeast to rapid shallow marine deposition on the northwest. The shallowness of the sea to the northwest is attested by the presence of the pelecypods Ostrea, Lopha, and Mytilus. The scarcity of megafossils to the northwest in the Twelvemile Canyon Member of the Arapien Shale is probably related to rapid subsidence accompanied by equally rapid sedimentation. 


\section{SYSTEMATIC DESCRIPTIONS}

Genus NUCULA Lamarck, 1799

Nucula sp.

Plate 1, figures 1-3

The genus Nucula has been found in the Jurassic of the western interior region only at several localities near Cedar City, Utah. At these localities it occurs in thin beds of yellow oolitic slightly sandy pebbly limestone lying from 230 to 255 feet above the base of the Carmel Formation. It is commonly associated with small oysters, crinoid columnals, and small gastropods. Less commonly it occurs with trigonias and Camptonectes.

The species is small, subtrigonal, and moderately convex. The umbones are prominent and are situated about one-third of the length of the shell from the anterior end. The ventral margin curves evenly into the anterior margin and more sharply into the posterior margin. The surface is marked by weak concentric growth lines. The interior of the shell has a taxodont hinge and simple palial line.

Figured specimens: USNM 131917.

Occurrences in southern Utah: USGS Mesozoic locs. 18213, 19428, 19432, 25672, 28461.

\section{Genus IDONEARCA Conrad, 1862}

\section{Idonearca? sp.}

Plate 1, figures $10-12$

This species is represented by five specimens that retain considerable shell material. The shell is moderate in size, inflated, equivalve, inequilateral, elongate, subrhomboidal in outline. The dorsal margin is straight and makes an angle of about $135^{\circ}$ with the nearly straight posterior margin. The anterior margin is regularly rounded. The ventral margin is slightly convex and rounds acutely into the posterior margin. The beaks are widely separated, incurved, fairly prominent, situated near the middle of the ligamental area, and about one-third of the length of the shell from the anterior end. A prominent angular ridge curves backward from the beak to the postero-ventral end. It is bordered dorsally by a deep depression and ventrally by a shallow depression. The surface of the cardinal area is marked by at least five chevron-shaped ligamental grooves, but the exact number cannot be ascertained. The hinge line is straight and about two-thirds as long as the length of the shell, inclined slightly obliquely to the hinge margin and is exposed on the anterior part of one specimen. The surface of the shell is ornamented with strong concentric growth lines. Radiating striae are not visible, but their absence may be due to corrosion. The figured speci- men has a length of $42 \mathrm{~mm}$, a height of $29 \mathrm{~mm}$, and a convexity of $28.5 \mathrm{~mm}$.

This species compares in shape with Cucullaea? elongata Warren (1932, p. 12, pl. 1, fig. 15) from beds of Bajocian age near Blairmore, Alberta, but has a more angular umbonal ridge, and the area above the ridge is more depressed. Idonearca? haguei Stanton (1899, p. 623 , pl. 73 , figs. 11-13) is much higher, is trigonal in outline, and has much more prominent umbones.

Figured specimen: USNM 131918.

Occurrences in southern Utah: USGS Mesozoic locs. 26307, $28468,28461$.

\section{Genus MODIOLUS Lamarck, 1799}

Modiolus subimbricatus (Meek)

Plate 1, figures 16-20

Modiola (Vulsella) subimbricata Meek, 1873, U.S. Geol. Survey Terr. (Hayden) 6th Ann. Rept., 1872, p. 472.

Volsella subimbricata (Meek). White, 1880, U.S. Geol. Survey Terr. (Hayden), 12th Ann. Rept., 1878, pt. 1, p. 145, 146, pl. 37, figs. 2 a-c.

Modiola subimbricata (Meek). Stanton, 1899, U.S. Geol. Survey Mon. 32, pt. 2, p. 617.

This species is characterized by its imbricating concentric sculpture. As noted by Meek (1873, p. 472), it is similar to Modiolus imbricatus (Sowerby) as illustrated by Morris and Lycett (1853, pl. 4, fig. 2) except " *** that its anterior ventral portion, in front of the umbonal ridges, is more prominent, and its posterior basal extremity more produced and narrowed."

Modiolus sphenoproratus Crickmay (1930, p. 56, pl. 7b) from the Bajocian Opuntia Formation of southwestern British Columbia appears to have a weaker umbonal ridge than M. subimbricatus (Meek), but it could be an immature representative of that species. M. rosii McLearn (1924, p. 53, pl. 8, fig. 1, pl. 9, fig. 12) is distinguished by its author from $M$. subimbricatus (Meek) by being larger, not as strongly arched, and less elongated posteroventrally. It appears, also, to have a longer hinge line.

Modiolus subimbricatus (Meek) is fairly common in the western interior region in beds of Bajocian to early Callovian age. The Geological Survey collections from central and southern Utah contain 31 specimens of the species.

The original collection studied by Meek was obtained from the Lower Canyon of the Yellowstone River about 2 miles south of Livingston, Mont. It contains 8 specimens of which 1 was illustrated by White (1880, pl. 37, fig. 2a). This specimen is herein designated the lectotype of Modiolus subimbricatus (Meek) and is re-illustrated.

Types: Lectotype USNM 131919; syntypes USNM 131920, 131921, 7818; plesiotypes USNM 131922, 131923. 
Occurrences in central and southern Utah: USGS Mesozoic locs. 963, 964, 7341, 11438, 11439, 12840, 15877, 16624, 17054, $27467,27470,28461-28463$, and 28491 .

\section{Modiolus isonemus (Meek)}

Plate 1, figure 22

Volsella scalprum var. isonema Meek, 1877, U.S. Geol. Explor. 40th Parallel (King), v. 4, pt. 1, p. 132, 133, pl. 12, figs. 4 , $4 \mathrm{a}$.

This species is characterized by its fine regularly spaced concentric ribs. M. subimbricatus (Meek) differs by having coarser imbricate ribs and a much more highly arched form. M. pertinuis (Meek and Hayden) (1858, p. 51; 1865, p. 86, pl. 3, figs. 5, 5a), whose type specimen is probably lost, has similar fine regular concentric ribbing but appears to differ by its less arcuate form, less prominent umbonal ridges, and less inflated anterior end.

The holotype was obtained from Weber Canyon in the Wasatch Range of north-central Utah, presumably from the Twin Creek Limestone. In central Utah the species is represented by one external and one internal mold from the Arapien Shale of San Pete County. The species has not been reported elsewhere from the western interior region.

Type: Holotype USNM 12545.

Occurrence in central Utah: USGS Mesozoic loc. 21450.

\section{Genus MYTILUS Linné, 1758}

Mytilus cf. M. whitei Whitfleld

Plate 1, figures 4-6

The genus Mytilus is represented in southern Utah by four internal molds that are similar in shape to $M$. whitei Whitfield (1877, p. $18 ; 1880$, p. 360 , pl. 5, figs. 9-12) from the Jurassic of the Black Hills but appear to lack the rugose irregular concentric ribs of that species. Their smoothmess, however, may be due to the absence of shell material.

Figured specimens: USNM 131924, 131925.

Occurrences in central and southern Utah: USGS Mesozoic locs. $6941,15551,25675$, and 28477.

\section{Genus GERVILIIA Defrance, 1820}

\section{Gervillia montanaensis Meek}

Plate 1, figure 21

Gervillia montanaensis Meek, 1873, U.S. Geol. Survey Terr. (Hayden), 6th Ann. Rept., 1872, p. 472.

Gervillia sparsilirata Whitfield, 1876, in Ludlow, Wm., Report of a reconnaissance from Carroll, Montana Territory *** to the Yellowstone National Park and return $* * *$ p. 142, pl. 2 , fig. 8 .

Gervillia montanaensis Meek. White, 1880, U.S. Geol. Survey Terr. (Hayden), 12th Ann. Rept., 1878, pt. 1, p. 145 pl. 37 , figs. 1a, b.
Gervillia montanaensis Meek. ' Stanton, 1899, U.S. Geol. Survey Mon. 32, pt. 2, p. 617.

This species is represented in southern Utah by an external mold at USGS Mesozoic loc. 26308 and is probably represented by internal molds at Mesozoic locs. 20359 and 28464.

\section{Genus ISOGNOMON Solander, 1786}

Isognomon perplana (Whitfleld)

Plate 1, figures 32-34

Myalina? (Gervillia) perplana Whitfield, 1876, in Ludlow, Wm., Report of a reconnaissance from Carroll, Montana Territory $* * *$ to the Yellowstone National Park and return $* * *$, p. 143, pl. 1, fig. 8.

?Mytilus whitei White (not Whitfield), 1880, U.S. Geol. Survey Terr. (Hayden), 12th Ann. Rept. 1878, pt. 1, p. 147, pl. 37, fig. 9a.

The holotype is a left valve that lacks part of the ventral margin and is not sufficiently well preserved to show the amount of prolongation of the umbones. It was obtained near the south end of the east side of the Bridger Range about 6 miles north of Fort Ellis, Mont. It is reported to have been obtained from about 60 feet of "firm blue compact limestone" and to be associated with other Jurassic fossils that include Gryphaea planoconvexa Whitfield. Actually the holotype is embedded in a brownish-gray fine-grained limestone matrix that matches very well with limestone in the middle part of the Piper Formation exposed on the north side of Bridger Creek at the south end of the Bridger Range.

The best preserved specimens of Isognomon perplana (Whitfield) have been found in beds of Bajocian age in and near Yellowstome National Park. These show that the shell is high, elongated obliquely, and is gently inflated in the umbonal region. The umbones are fairly prolonged. The anterior margin is gently concave in its dorsal half and gently convex in its ventral half. The ventral margin is narrowly rounded. The posterior margin is nearly straight and forms an obtuse angle with the hinge line. The surface of the shell bears variably spaced irregularly weak growth lamellae. The ligamental area bears narrow vertical ligamental pits.

Isognomon perplana (Whitfield) is similar in shape and ornamentation to Isognomon promytiloides Arkell (1933, pl. 27, figs. 2, 3, 3a, text figs. 49-52) from the Upper Jurassic of Europe, but its umbones appear to be less projected. I. weelaupensis McLearn (1926, p. 93 , pl. 19, figs. 1,2) from the Bajocian beds of western British Columbia likewise has much more projected umbones as well as more vigorous ribbing.

The genus Isognomon is moderately common in beds of Bajocian age in the western part of the western 
interior region. Most of the specimens, however, are fragmentary molds that have been assigned commonly to Gervillia or Perna and are probably not identifiable specifically. The Geological Survey collections from the Jurassic beds of southern Utah contain 33 specimens of Isognomon (pl. 1, figs. 30,31, 35, 36) of which all are fragmentary. They show resemblances to Isognomon perplana (Whitfield) and possibly belong to that species. Several show the narrow vertical ligamental pits that are characteristic of the genus.

Types: Holotype, Yale Peabody Museum 21280; plesiotypes USNM 131926, 131927. Figured specimens of Isognomon cf. $I$. perplana (Whitfield), USNM 8177, 131928, 131929.

Occurrences of Isognomon in southern Utah: USGS Mesozoic locs. $963,10116,10436,12581,16202,16624,17054,19422$, 19429, 19431, 20358, 25680,27469, 27591, 28461, 28469, 28473, and 28496.

\section{Genus INOCERAMUS J. Sowerby, 1814}

\section{Inoceramus crassalatus White}

Plate 1, figures 24, 25

Inoceramus crassalatus White, 1877, U.S. Geog. Surveys West 100 Meridian (Wheeler), Rept., v. 4, pt. 1, p. 166, pl. 13 , figs. $4 \mathrm{a}-\mathrm{c}$.

The original description of the species is as follows:

Shell rather small, thin; valves subequal, moderately convex, subovate or obscurely tetrahedral in marginal outline; hinge-line rather short, forming an angle with the axis of the shell of about seventy degrees; beaks small, not very prominent. Left valve more capacious than the right, having an indistinct auricular furrow extending from just behind the beak to the postero-basal border, obscurely defining a thick posterior wing; but the right valve has little or no trace of a similar furrow.

Surface of both valves marked by the usual lines of growth, and also by more or less numerous, slightly raised, concentric folds.

Greatest length of an average example in the collection, about thirty millimeters; thickness, both valves together, about fifteen millimeters.

This species is characterized by the valves being markedly unequal, by the left valve bearing a conspicuous furrow, and by the concentric ornamentation consisting of both broad folds and fine ribs. The type collection includes four specimens which, judging by their size, are probably immature.

This species is of particular interest because it is represented to date only by the four type specimens, because no other specimen of Inoceramus has been found in the Jurassic of central and southern Utah, and because Inoceramus is extremely rare in the Jurassic of the western interior region south of central Montana. Furthermore, the type specimens bear weak concentric ribs and folds that are quite different from the coarse ribs on specimens of Inoceramus from the Jurassic of Alberta (McLearn, 1924, p. 41, pl. 3, fig. 9; Warren, 1932 , p. 15 , pl. 2, figs. 1-3) and northern Montana. These facts suggest the possibility that the type specimens of Inoceramus crassalatus White may be from Cretaceous instead of Jurassic rocks.

The specimen illustrated by White on his plate 13, figure $4 \mathrm{a}$ is herein selected as lectotype of $I$. crassalatus White.

Types: Lectotype USNM 131929; syntypes USNM 131930, 8585 .

Occurrence: The type lot is labeled "Jurassic North Fork of Virgin River, Utah." This would be in either northeastern Iron County or westernmost Kane County.

\section{Genus PINNA Linné, 1758}

\section{Pinna kingi Meek}

Pinna kingi Meek, Meek, 1877, U.S. Geol. Explor. 40th Parallel (King), v. 4, pt. 1, p. 131, 132, pl. 12, figs. 9, 9a.

Pinna kingi Meek. Stanton, 1899, U.S. Geol. Survey Mon. 32, pt. 2, p. 618.

Pinna kingi Meek. Veatch, 1907, U.S. Geol. Survey Prof. Paper 56, pl. 6, figs. 11, 11a.

This species appears to have much stronger ribs and growth lines than Pinna jurassica Whitfield and Hovey (1906, p. 392, pl. 44, pl. 45, fig. 1) from the Black Hills, South Dakota. The type specimens of $P$. jurassica Whitfield and Hovey occur in a slab of glauconitic sandstone suggestive of sandstones near the base of the Redwater Shale Member of the Sundance Formation. This member is correlated with the upper part of the Oxfordian stage. The type specimen of Pinna kingi Meek was obtained as float in Weber Canyon, Utah. Presumably it was derived from the Twin Creek Limestone. The species is fairly common in the western part of the western interior region in beds of Bajocian age. It is represented by five fragmentary specimens from southwestern Utah.

Occurrences in Utah: USGS Mesozoic locs. 18670, 25669, 26308 , and 28472.

\section{Genus CAMPTONECTES Meek, 1864}

\section{Camptonectes stygius White}

Plate 2, figures 1-10

Camptonectes stygius White, 1874, U.S. Geog. and Geol. Surveys West 100th Meridian (Wheeler), p. 23.

Camptonectes stygius White, 1877, U.S. Geog. Surveys West 100th Meridian (Wheeler), v. 4, pt. 1, p. 164, 165, pl. 13, figs. $2 \mathrm{a}-\mathrm{c}$.

Camptonectes stygius White. Butler and others, 1920, U.S. Geol. Survey Prof. Paper 111, pl. 10, fig. 19.

The shell is medium in size, suborbicular, moderately convex. The left valve is more convex than the right. The hinge line is about half as long as the width of the shell. The auricles are moderate in size for the genus and are covered with fine growth lines. The anterior auricles are much larger than the posterior auricles. A deep byssal notch occurs beneath the right anterior auricles. 
The surface of the shell is covered mostly with fine concentric growth lines. Faint radiating striae are barely visible near the margins of the best preserved shells and appear to be slightly stronger on left valves than on right valves.

This species is very common in the western interior region in beds of Bajocian to early Callovian age. It is represented by several hundred specimens in the Geological Survey collections from central and southern Utah.

The specimen illustrated by White (1877, pl. 13, fig. 2a) is herein selected as lectotype of the species. The type specimens are from two widely separated localities described as " $* * *$ east of Aquarius Plateau, fifteen to twenty miles south of Dirty Devil River, and also at the North Fork of Virgin River, Utah."

Types: Lectotype USNM 131931; syntypes USNM 131932131934, 8580; plesiotypes USNM 131935-131938.

Occurrences in central and southern Utah: USGS Mesozoic locs. 963, 964, 5261, 7341, 7890, 7891, 8597, 10098, 10116, 10436, 11137 $11438,11439,11445,12555,12841,13756,16202,16700,16701$, $16703,17054,17269,17353,17412,17462,17494,18281,18282$, $19422,19424,19426,19429,20358,20364,21448,22656,24258$, $24260,25670,25678,25679,25682,25686,25826,25827,27465$, $27466,27468,28461,28462,28472,28491$, and 28495 .

\section{Camptonectes platessiformis White}

Plate 2, figures 11-14

Camptonectes platessiformis White, 1880, U.S. Geol. Survey Terr. (Hayden) 12th Ann. Rept., 1878, pt. 1, p. 143, 144, pl. 37, fig. 5a.

Camptonectes platessiformis White. Stanton, 1899, U.S. Geol. Survey Mon. 32, pt. 2, p. 615.

Camptonectes extenuatus Whitfield (not Meek and Hayden). Whitfield, 1880, U.S. Geog. and Geol. Survey Rocky Mtn. Region, p. 353 , pl. 4 , fig. 4 .

This species is subovate in shape and higher than wide. The hinge line is about two-thirds as wide as the shell. The auricles are large for the genus and have the shape of right-angle triangles. The anterior ear is considerably larger than the posterior ear. The surface of the shell is marked by fairly coarse fan-wise radiating crenulated obliquely curved branching ribs. The ribs are much wider than the interspaces, are flattopped and broad on the umbones but become broadly rounded and slightly narrower toward the margins of the shell. The interspaces are punctate. Fine concentric growth lines produce a cancellate pattern near the margins of the larger shells.

This species may be distinguished from Camptonectes bellistriatus (Meek) (Meek and Hayden, 1865, p. 77, 78 , text figs. a-d) by its more slender shape, much smaller size in the adult, much longer hinge line, and broader coarser ribs. C. distans Stanton (1899, p. 614, pl. 72, fig. 13) differs by having much broader ribs and a broader shell (pl. 2, figs. 15, 16). It is associated with $C$. platessiformis White in the western interior region but is much less common. C. platessiformis White in the Yellowstone National Park area of Wyoming and Montana occurs in beds that contain ammonites of Bajocian to early Callovian age. $C$. bellistriatus (Meek) occurs higher stratigraphically in beds of Oxfordian age, such as the Stump Formation and the Upper Sundance Formation.

C. platessiformis White is represented in central and southern Utah by about 60 specimens of which most are fragmentary and poorly preserved.

Types: Holotype USNM 8179; plesiotypes USNM 131939, 131940.

Occurrences in central and southern Utah: USGS Mesozoic locs. $963,964,11438,15878,16395,16624,17054,17198,17462$, $17463,18670,18922,19431,21450,24258,24260,25669,25672$, $25673,25675,25682,26308,26309,27469,27474,25682,28461$, $28472,28477,28479$. Specimens comparable with $C$. platessiformis White occur at USGS Mesozoic locs. 7341, 12555, 13529, 18671, 27466, 27475, and 27476.

\section{Genus PLICATULA Lamarck, 1801}

Plicatula sp.

Plate 1, figures 13-15

The genus Plicatula is represented in central and southern Utah by 18 specimens that show considerable variation in fineness of ribbing and in spinosity. Finely ribbed specimens occur at U.S. Geological Survey Mesozoic localities 7341, 10116 and 13756. More coarsely ribbed specimens occur at U.S. Geological Survey Mesozoic localities 12555, 17462, 21446, 21448, $25672,27470,28461$, and 28495 . The coarser ribbed specimens resemble $P$. peregrina d'Orbigny from the Callovian beds of India (Cox, 1952, p. 40, pl. 4, figs. 2-6).

Figured specimens: USNM 131941, 131942.

\section{Genus ANOMIA Linné, 1758}

\section{Anomia? sp.}

Plate 1, figure 23

The genus is represented in the Carmel Formation of southern Utah by five specimens that are very small, ovate in outline, and highly convex. The beak appears to be near the dorsal margin, but its tip is broken. The surface bears broad faint concentric folds and microscopic radiating flexuous striae.

Figured specimen: USNM 131943.

Occurrence in southern Utah: USGS Mesozoic locs. 18922 and 28472 . 


\section{Genus LIMA Bruguiere, 1797 \\ Subgenus PLAGIOSTOMA J. Sowerby, 1814}

Lima (Plagiostoma) occidentalis Hall and Whitfield

Plate 2, figures $17-20$

Lima occidentalis Hall and Whitfield, 1877, U.S. Geol. Explor. 40th Parallel (King), v. 4, pt. 2, p. 292, 293, pl. 7, fig. 23. Lima occidentalis Hall and Whitfield. Butler and others, 1920, U.S. Geol. Survey Prcf. Paper 111, pl. 10, fig. 16 .

This species was based on a single left valve from Jurassic beds exposed in the Flaming Gorge southeast of Manila in northeast Utah. The valve lacks most of the umbonal region. It is subovate in outline and slightly oblique. It has a height of about $45 \mathrm{~mm}$, a length of $42 \mathrm{~mm}$, and a convexity of about $12 \mathrm{~mm}$. The anterior umbonal ridge is well defined. The lunule is fairly deep and long. The posterior auricle is small, only partly preserved, bears fine radial ribs, and merges with the body of the shell. The ornamentation of the valve consists of about 60 rounded radial ribs that are about twice as wide as the intervening grooves. Both ribs and grooves near the ventral margin are crossed by very fine concentric growth striae that are most conspicuous in the grooves. The grooves are not punctate on the parts of the shell that are preserved.

Other specimens of this species show that punctae are present in grooves on the umbonal region but that ventrally the punctae are replaced by fine striae. Also, the width of the radial ribs in some specimens may be three times greater than that of the grooves. The anterior auricle is much smaller than the posterior auricle.

This species is uncommon in the western interior region outside of Utah. A few specimens have been found, however, in western Wyoming and southeastern Idaho in beds of Bajocian to early Callovian age. In Utah the species is moderately common in the lower part of the Carmel Formation. It is represented by 37 specimens from central and southern Utah.

Types: Holotype USNM 12539; plesiotypes USNM 131944 131946.

Occurrences in central and southern Utah: USGS Mesozoic loes. $5278,6941,16624,16700,17349,19429,26309,27469,27474$, $27475,28461,28475,28477$, and 28479. Fragments that possibly belong to the species occur at Mesozoic locs. 17495, 18213 , and 27476 .

\section{Lima (Plagiostoma) ziona Imlay, n. sp.}

Plate 2, figures 21-26

This species differs from Lima (Plagiostoma) occidentalis Hall and Whitfield by having only about half as many ribs. Also, the ribs are much broader, are flat topped on the umbonal region, and tend to split toward the ventral margin. The left valve of the holotype has about 30 ribs. The left valve of the larger paratype, shown on plate 2, figure 26, has 32 ribs of which 7 ribs near the posterior auricle are much smaller than the others. The ornamentation near the beak consists of widely separated rows of punctae. Ventrally on the umbones these rows of punctae develop into narrow punctate grooves that separate very broad flat-topped ribs. On the largest specimens the punctae are gradually replaced ventrally by fine transverse markings, the radial grooves become a little broader and deeper, and the ribs become broadly rounded. The left valve of the holotype has a length of $42 \mathrm{~mm}$ and a width of $40 \mathrm{~mm}$. The largest paratype has a length of $48 \mathrm{~mm}$ and a width of $43 \mathrm{~mm}$.

This species is represented by 35 specimens from central and southern Utah. It is also fairly common in northern Utah but has not yet been identified elsewhere in the western interior region. Most of the specimens in the Geological Survey collections are from Washington and Iron Counties and the western part of Kane County. All the specimens from these counties are from parts of the Carmel Formation that have furnished Lima (Plagiostoma) occidentalis Hall and Whitfield, but surprisingly the two species have been found in association only near Cedar City (USGS Mesozoic loc. 27469). This suggests that the two species lived under slightly different environmental conditions.

Types: Holotype USNM 131947; paratypes USNM 131948131950.

Occurrences in central and southern Utah: USGS Mesozoic locs. 961,12749 , 18281, 25672, 25675, 25682, 26308, 27467, 27469, 28471 , and 28491 .

\section{Genus CTENOSTREON Eichwald, 1862}

\section{Ctenostreon sp.}

This genus is represented in southern Utah by three fragments that are similar in size and in strength of ribbing to Ctenostreon gikshanensis McLearn (1926, p. 91, pl. 19, figs. 3, 4) from Bajocian beds in British Columbia.

Occurrence in southern Utah: USGS Mesozoic locs. 18670 and 26309.

Genus OSTREA Linné, 1758

Subgenus LIOSTREA Douville, 1904

Ostrea (Liostrea) strigilecula White

Plate 1 , figures $26-29$

Ostrea strigilecula White, 1877, U.S. Geog. Surveys West 100th Meridian (Wheeler), v. 4, pt. 1, p. 163, 164, pl. 13, figs. $3 \mathrm{a}-\mathrm{d}$.

Ostrea strigilecula White. Whitfield, 1880, U.S. Geog. and Geol. Survey Rocky Mtn. Region, p. 348, 349, pl. 3, figs. 8-12. Ostrea strigilecula White, 1884, U.S. Geol. Survey 4th Ann. Rept., p. 289, 290, pl. 35, figs. 9, 11. 
Ostrea strigilecula White. Stanton, 1899, U.S. Geol. Survey Mon. 32, pt. 2, p. 610.

?Ostrea strigilecula White. Logan, 1900, Kansas Univ. Quart., v. 9 , no. 2 , ser. A, p. 120,121 , pl. 28 , figs. $1-6$.

Ostrea strigilecula White. Butler and others, 1920, U.S. Geol. Survey Prof. Paper 111, pl. 10, fgs. 8, 9.

This species is small, subovate in outline, and the axis of shell is curved slightly posteriorly. The right valve is fiat to slightly concave. In some right valves the umbonal part is slightly convex and the ventral part slightly concave. The left valve is strongly swollen, much larger than the right valve, bears ordinary lines of growth, and is marked by an attachment scar of variable size. Many left valves have a weak winglike expansion of the posterior margin. The left valves do not bear radiating plications, although White $(1877, \mathrm{p}$. 164) thought that there were a few faint indications of plications.

The primary types were obtained from the Carmel Formation south of the Dirty Devil River in eastcentral Utah, presumably from Wayne County. The species has been found subsequently in enormous numbers at many places in the Carmel Formation of Utah, and the name has been applied to similar small nondescript hump-shaped oysters from the marine Jurassic beds throughout the western interior region.

The specimen figured by White (1877) on his plate 13 , figures $3 . \mathrm{a}-\mathrm{c}$ is herein selected as lectotype of Ostrea strigilecula White.

Types: Lectotype USNM 131951; syntypes USNM 131952 8581; plesiotype USNM 131953.

Occurrences: As noted on tables 3-8.

Genus LOPHA Bolten, 1798

Lopha aff. L. engelmanni (Meek)

Plate 1, figures 7-9

Twenty-one specimens of a small ovate oyster have been obtained in Iron and Kane Counties in southern Utah. These specimens are characterized by a few broad irregular radial plications that are somewhat stronger on the left valve than on the right valve. The left valve is slightly to moderately convex; the right valve is flat to slightly concave.

This species differs from "Ostrea" dowlingi McLearn (1924, p. 43, pl. 3, figs. 6-8) from the Callovian beds of Alberta by being more elongate and by having fewer and coarser ribs. Its rib pattern is more similar to that on "Ostrea" engelmanni Meek (1860, p. 311; Meek and Hayden, 1865 , p. 73 , 74, figs. A, B; Meek, 1876 , p. 355 , pl. 3, fig. 6; White, 1884 , p. 289 , pl. 34 , figs. 3 , 4) from the Upper Sundance Formation of eastern and central Wyoming, but it is much smaller than that species.

Figured specimens: USNM 131954, 131955

Occurrence in southern Utah: USGS Mesozoic locs. 5261, 5263, 17353, 18281, 18660, 28462, and 28494.

\section{Genus TRIGONIA Bruguière, 1789}

\section{Trigonia americana Meek}

Plate 3, figures 8-13

Trigonia americana Meek. 1873, U.S. Geol. Survey Terr. (Hayden) 6th Ann. Rept., 1872, p. 472.

Trigonia americana Meek. White, 1880, U.S. Geol. Survey Terr. (Hayden), 12th Ann. Rept., 1878, pt. 1, p. 148, pl. 38, figs. 1a, b.

Trigonia americana Meek. Stanton, 1899, U.S. Geol. Survey Mon. 32, pt. 2, p. 618.

The original description by Meek (1873, p. 472) is as follows:

A fine species of the type of $T$. costata of the Old World, but differing from that and the other allied forms, in having the radiating costae of the corselet, or posterior dorsal region, all of uniform size.

The description by White (1880, p. 148) is as follows:

Shell subtetrahedral in marginal outline, gibbous in front of the umbonal ridge and compressed behind it; the margin from the beak all the way to the postero-basal angle forming a continuous and almost regular curve; posterior margin nearly straight, truncating the shell downward and backward; dorsal margin gently concave; beaks somewhat elevated and having the appearance of being directed slightly backward; umbonal ridge forming a somewhat prominent crenulated carina, which very gradually increases in size towards and ending at the posterobasal angle. Surface in front of the umbonal ridge marked by 15 to 18 sharply-raised concentric varices, which become suddenly obsolete just before reaching the umbonal ridge. The surface behind that ridge is marked by numerous raised, radiating, finely crenulated lines, which are nearly uniform in size; but the spaces separating the five and six lower ones are a little wider than the others.

Length from front to posterior margin, $34 \mathrm{~mm}$; heights from base to beaks, $31 \mathrm{~mm}$.

Both White and Meek noted that T. americana Meek was characterized by the uniform character of the radiating riblets on the area posterior to the marginal carina. The area is divided into two parts, however, by a weak groove. The general appearance of the species is similar to that of $T$. denticulata Agassiz as figured by Lycett (1877, p. 152, pl. 29, figs. 1-3) except for somewhat sparser ribbing. In this respect it resembles T. costata Sowerby (Lycett, 1877, pl. 30, figs. 3, $3 \mathrm{a}, \mathrm{b}$, 6 ; Cox, 1952 , p. 109 , pl. 12 , figs. $3,4,7$ ), but its posterior area bears much finer radial riblets and is not divided by a prominent median carina, its posterior carina bears much finer crenulations, and its shell is much less elongate. T. guhsani McLearn (1926, p. 94, pl. 20, figs. 2,3), from middle Bajocian beds in British Columbia, differs from $T$. americana Meek by being much larger, by having denser concentric ribs, fewer riblets on the posterior area, and by being more elongated posteriorly.

This species is fairly common in the western interior region in beds of Bajocian to early Callovian age. The 
type specimens are from the middle of the Piper Formation exposed at the mouth of Yellowstone Canyon about $2 \frac{1}{2}$ miles south of Livingston, Mont. The beds from which they were obtained have furnished the Bajocian ammonite Zemistephanus (USGS Mesozoic locs. 19214, 15635). Near Lower Slide Lake, Teton County, Wyo. (Imlay, 1956a, p. 70), Trigonia americana Meek occurs with ammonites of Bajocian to Callovian age. The species is represented in the U.S. Geological Survey collections from the Carmel Formation of Utah by 9 adult specimens and probably also by 9 immature specimens.

The specimen illustrated by White $(1880$, pl. 38 , fig. 1) is herein selected as lectotype of the species.

Types: Lectotype USNM 131956; syntypes USNM 131957, 7794, 7819; plesiotype USNM 131958.

Occurrences in central and southern Utah: Carmel Formation at USGS Mesozoic locs. 7341, 24258, 25669, 25684, 27469. The species is probably represented by some small immature specimens at Mesozoic locs. 16624, 25670, 25672, 25673, and 26308.

\section{Trigonia elegantissima Meek}

Plate 3, figures 1-7

Trigonia elegantissima Meek, 1873, U.S. Geol. Survey Terr. (Hayden) 6th Ann. Rept., 1872, p. 474.

Trigonia elegantissima Meek. Stanton, 1899, U.S. Geol. Survey Mon. 32, pt. 2, p. 619, pl. 73, fig. 2.

The original description by Meek (1873, p. 474) is as follows:

A small species of the type of $T$. costata, but having the concentric or horizontal costae on the sides of the valves very delicate, closely arranged, and but slightly larger than the radiating ones on the posterior dorsal region, or corselet. The valves are rather compressed, about one-fourth longer than wide, and have the posterior umbonal slopes acutely angular.

The description by Stanton (1899, p. 619) is as follows:

Shell small, subtrigonal in outline, moderately convex, with prominent, acute, recurved beaks; posterior umbonal ridge prominent, angular, and curved; anterior end broadly rounded; posterior end subangular below at the extremity of the umbonal ridge and forming a convex slope to the beak above; escutcheon not distinctly marked; posterior area depressed and bearing numerous equal, fine radiating lines; remainder of surface with regular, closely arranged, small concentric ribs that show a tendency to bend downward toward the front.

Length of figured specimen, $21 \mathrm{~mm}$; height, $14 \mathrm{~mm}$; convexity of single valve, $4 \mathrm{~mm}$.

This species is closely related to $T$. americana, from which it differs in outline, and more especially in having much smaller and more numerous concentric ribs. In specimens of $T$. americana no larger than the type of this species the spaces between the ribs are at least a millimeter wide.

Trigonia elegantissima Meek differs from T. americana Meek, with which it is commonly associated, by its less erect form, much smaller adult size, much finer and denser ribbing, and by its ribbing becoming denser ventrally instead of sparser. The species shows some variation in the coarseness of its ribbing, but even the most coarsely ribbed specimens have much finer ribbing than on T. americana Meek. The downward bending of the concentric ribs near the anterior margin, described by Stanton, occurs on only some of the more finely ribbed variants. The marginal carina is nearly smooth, but on the best preserved specimens bears faint crenulations. The radiating lines or fine riblets on the area are crossed by finer threads.

The specimen from the Devils Slide area, Montana, illustrated by Stanton (1899, p. 73, fig. 2) is the most finely ribbed of the specimens available. Somewhat less finely ribbed specimens from the north side of Lower Slide Lake, Teton County, Wyoming, are herein illustrated for comparison (pl. 3 , figs. 4,5 ). In collections from the Carmel Formation of Utah, most of the specimens of $T$. elegantissima Meek represent medium to coarsely ribbed variants, but some are as finely ribbed as the specimen illustrated by Stanton.

Among American species, T. elegantissima Meek is most similar to Trigonia poststriata Whitfield and Hovey (1906, p. 396, pl. 48, figs. 4-6) obtained 4 miles southwest of Hulett, Wyo., but differs by being considerably smaller, less elongate posteriorly, and by its concentric ribs remaining distinct instead of fading on the posterior part of the area.

Among foreign species, T. elegantissima Meek is similar in shape and fineness of ribbing to T. hemisphaerica Lycett (1877, p. $174-178$, pl. 31 , figs. 4-8) from the Inferior Oolite of England, differing mainly by its much smaller size, sparser ribbing near the beak, and weaker crenulations on the area and marginal carina.

Trigonia elegantissima Meek in the western interior is associated with such species of the Trigoniidae as T. americana Meek, Myophorella (Promyophorella) conradi (Meek and Hayden), and Vaugonia montanaensis (Meek) but is much less common. It is represented in the Carmel Formation of Utah by only 15 specimens and is similarly poorly represented in collections from Montana and Wyoming. Its range, based on associated ammonites found with it in Wyoming and Montana, is from Bajocian to basal Callovian. For example, in the Jurassic section near Lower Slide Lake, Wyoming (Imlay, 1956a, p. 70, 71), it ranges from the lower part of unit 10 into unit 19. In unit 10 it is associated with an ammonite of Bajocian age (USGS Mesozoic loc. 20967). In unit 19 it is associated with the ammonites Arcticoceras and Cadoceras of earliest Callovian age (USGS Mesozoic loc. 22112). A similar range is shown by occurrences of the species in northcentral Wyoming and south-central Montana (Imlay, 1956 b, p. 576, 577, 583, 584, 591).

Types: Plesiotypes USNM 28935, 131959-131964. The holotype was never figured. The specimen mentioned by Stanton 
(1899, p. 619) as probably being the type has not been located. Occurrences in central and southern Utah: Carmel Formation at USGS Mesozoic locs. 11439, 12555, 15878, 19422, 20351, 25686 , and 27472 .

\section{Genus VAUGONIA Crickmay, 1930}

\section{Vaugonia conradi (Meek and Hayden)}

Plate 3, figures 14-21

Trigonia conradi Meek and Hayden, 1860, Acad. Nat. Sci. Philadelphia Proc. 1860, p. 183, 184.

Trigonia conradi Meek and Hayden, 1865, Smithsonian Inst., Smithsonian Contr. Knowledge, v. 14, no. 172 , p. 83 , 84, pl. 3, fig. 11 .

Not Trigonia quadrangularis Hall and Whitfield, 1877, U.S. Geol. Explor. 40th Parallel (King), v. 4, pt. 2, p. 293, 294, pl. 7, fig. 22.

Trigonia quadrangularis Hall and Whitfield. Veatch, 1907, U.S. Geol. Survey Prof. Paper 56, pl. 6, fig. 8.

Not Trigonia quadrangularis Hall and Whitfield. Butler and others, 1920, U.S. Geol. Survey Prof. Paper 111, pl. 10, fig. 18.

Not Trigonia quadrangularis Hall and Whitfield. Mansfield, 1927, U.S. Geol. Survey Prof. Paper 152, pl. 31, fig. 18.

The original description of the species (Meek and Hayden, 1860, p. 183, 184) is as follows:

Shell rather small, subtrigonal, moderately convex; anterior side truncate; base rounded; posterior side sloping obliquely from the beaks above, and apparently vertically truncate at the extremity; beaks elevated, narrow, incurved, and located in advance of the middle; posterior umbonal slopes distinctly angular; surface ornamented by rather small, obscure concentric costae, which on the posterior side of the valves, descend at first perpendicularly, after which they are deflected forward parallel to the basal and anterior borders. Length and height, each about 0.97 inch; convexity 0.58 inch.

The type specimen, obtained from the southwest base of the Black Hills, is preserved in a fine-grained light grayish-yellow sandstone and is associated with Trigonia americana Meek and Myophorella (Promyophorella) cf. M. montanaensis (Meek).

The holotype is so corroded that the ornamentation is not well shown. Much better preserved specimens have been obtained subsequently in the southwestern part of the Black Hills from the upper part of the Canyon Springs Sandstone Member of the Sundance Formation (USGS Mesozoic locs. 20499, 20497), from the same member in the Hartville uplift in southeastern Wyoming (USGS Mesozoic loc. 20501), and from equivalent oolitic limestones near the base of the Sundance Formation near Cody, Wyoming (USGS Mesozoic loc. 20362). These are the beds characterized by Arcticoceras codyense Imlay. The species has also been found at many places in western Wyoming, southeastern Idaho, and western Montana in beds of Bajocian age containing such ammonites as Zemistephanus and Stemmatoceras.
Some of the specimens from these areas show that the species had a shape and ornamentation similar to that on Vaugonia veronica Crickmay (1930, p. 53, pl. 7f) and V. mariajosephinae Crickmay (1930, p. 54, pl. 7g) from the Middle Jurassic of Canada. The shape is more elongate posteriorly than is suggested by the holotype because the posterior end of the holotype is not preserved. The ribs on the disk form a sharp $V$. The ribs on the anterior part of the disk are high, thin, widely spaced, nontuberculate, and parallel to the ventral margin. The ribs on the posterior part of the disk are fairly thick, are weakly tuberculate transversely, and are vertical. During growth, the $V$ formed by the junction of these vertical and nearly horizontal ribs tends to become more acute and eventually broken. Most shells have 1 or 2 more horizontal ribs than vertical ribs.

The marginal carina is sharp and weakly crenulated. The inner carina is less sharp. The area is about one-third as wide as the disk, is crossed transversely by weak riblets, and is marked medially by a groove dorsal to a weak carina. The escutcheon is narrow, depressed, and nearly smooth.

Vaugonia conradi (Meek and Hayden) shows some variation in the density and strength of the ribbing on the disk and in the sharpness of the $V$ formed by the two sets of ribs on the disk. The specimens from the beds of Bajocian age in the Yellowstone National Park area are, in general, smaller and have denser ribbing (pl. 3, figs. 20, 21) than those in beds of Callovian age in the lower part of the Sundance Formation, although the specimen figured by Veatch (1907, pl. 6, fig. 8) from Bajocian beds west of Nugget, Wyo., is closely similar to specimens from the lower part of the Sundance Formation. The specimens from the Carmel Formation of central and southern Utah show similar variations in size and ribbing.

Most specimens of $V$. conradi (Meek and Hayden) in the Geological Survey collection have been misidentified as V. quadrangularis (Hall and Whitfield) (1877, p. 293, pl. 7, fig. 22) whose holotype is a fragmentary external mold preserved in a matrix of oolitic limestone obtained near Como Bluff, Wyo. Other specimens of $V$. quadrangularis have been found at that place by Pipiringos $(1957$, p. 23, 50) in an oolitic bed at the base of the Redwater Shale Member of the Sundance Formation. The holotype is a left valve that lacks part of its anterior margin (pl. 3, fig. 22). The remainder of the holotype indicates that the species is similar to $V$. conradi (Meek and Hayden), but differs by having broader, lower, and more closely spaced ribs on its disk. Such ribs are well shown on a specimen illustrated by Butler and others (1920, pl. 10, fig. 18) and Mansfield (1927, pl. 31, fig. 18) from the Stump Formation near Bear River City, Wyo. (Schultz, 1914, 
p. 53), and contrast markedly with the high thin widely spaced ribs on $V$. conradi (Meek and Hayden).

Similarly, $V$. sturgisensis Whitfield and Hovey (1906, p. 394-396, pl. 47, 48, figs. 1-3, 7) from the Black Hills of Wyoming and South Dakota differs from $V$. conradi (Meek and Hayden) by having broad low closely spaced ribs on its disk, by being more elongate posteriorly, and by attaining much larger size. Also, it occurs at a higher stratigraphic position. The largest specimen illustrated by Whitfield and Hovey (1906, pl. 48, fig. 7) is from the Redwater Shale Member of the Sundance Formation. The other specimens illustrated are probably from the Hulett Sandstone Member of the Sundance Formation.

Much closer resemblances to $V$. conradi (Meek and Hayden) are borne by the Canadian species $V$. veronica Crickmay (1930, p. 53, pl. 7f) which, judging from the holotype, appears to have somewhat stronger transverse ribbing on the area and a less acute $V$ formed by the ribs on the disk.

Compared with $V$. conradi (Meek and Hayden), $V$. praelonga (Gottsche) (1878, p. 26, pl. 6, figs. 2a, b) from the Bajocian of Argentina appears to be more elongate and to have stronger tubercles on the vertical ribs on the posterior part of the disk. $V$. v-costata (Lycett) (1874, p. 66, pl. 13, fig. 5, pl. 15, figs. 1-4) from the Inferior Oolite of England appears to develop the $V$ on its disk ribs at a later growth stage.

This species is represented by about 210 specimens in collections from central and southern Utah.

Types: Holotype USNM 212; plesiotypes USNM 131965131971.

Occurrences in central and southern Utah: USGS Mesozoic locs. $5268,6281,6282,10116,11137,12556,12570$, 12581, 12835, $12840,13532,16202,19432,20351,20356,20358,20359,22656$, $24259,25669,25670,25671,25673,25681,25684,25685,25686$, $26307,26309,28463,28468,28470,28472,28473,28480,28492$, 28493 . Immature or fragmentary specimens that possibly belong to $V$. conradi (Meek and Hayden) occur at Mesozoic locs. 16395, $21448,24260,25687,27469,27472,27475$, and 28491.

\section{Vaugonia aff. V. conradi (Meek and Hayden)}

Plate 3, figure 37

One specimen from Emery County, Utah, differs from $V$. conradi (Meek and Hayden) by an abrupt geniculation of the ribs in the anterior part of the disk. This results in the formation of a $V$ that is much more acute than on $V$. conradi but resembles that on $V$. (Hijitrigonia) geniculata Kobayashi and Mori (1955, p. 84, pl. 4, figs. 4-9). The Utah specimen differs, however, by lacking tubercles and by having widely spaced ribs on the anterior part of the disk.

Figured specimen: USNM 131972.

Occurrence in Utah: USGS Mesozoic loc. 13529
Vaugonia utahensis Imlay, n. sp.

Plate 3, figures 23-26

The shell is subtrigonal, moderately convex, elongated posteriorly. The umbo is located about onethird of the length of the shell from the anterior end. The disk is ornamented with ribs that form a $V$ near its posterior side. The ribs on the posterior side of the disk are vertical, thicken considerably ventrally, and are finely crenulated transversely. The ribs on the anterior side of the disk are irregularly fine, closely to moderately spaced, wavy to geniculate, apparently nontuberculate, tend to fade out near the middle of the disk, outnumber the vertical ribs 2 or 3 to 1 , and become very crowded and irregular near the ventral margin. The marginal carina is blunt. The area is about one-fourth as wide as the disk, is marked by a median groove, and is crossed transversely by striae. The escutcheon is depressed, is about half as wide as the area, and is crossed obliquely by striae.

The holotype, a right valve, is $25 \mathrm{~mm}$ long. $20 \mathrm{~mm}$ high, and $8 \mathrm{~mm}$ thick.

This species is distinguished from $V$. conradi (Meek and Hayden) by having much denser ribbing on the anterior side of the disk, by the anterior ribs being much more numerous than the posterior ribs of the disk, by having a blunt instead of a sharp marginal carina, and by having much weaker transverse markings on the area. The character of the ribbing on the disk of the Utah species shows some resemblance to that of Vaugonia (Hijitrigonia) kojiwa Kobayashi and Mori (1955, p. 85, pl. 4, figs. 2a, b, 3a, b) from the Lower Jurassic of Japan, but the marginal carina is blunt and rounded instead of prominent, and the shell is much more elongate posteriorly.

This species has been found only in southwest Utah and mostly in Kane, Iron, and San Pete Counties. About 85 specimens are available in the collections of the Geological Survey.

Types: Holotype USNM 131973; paratypes USNM 131974, 131975

Occurrences in Utah: USGS Mesozoic locs. 963, 16624, 17030, $17353,17494,20356,25672,26309,27473$. The species probably occurs at Mesozoic locs. 11439, 19429, 25683, 25685, 25826, and 28461 .

Genus MYOPHORELLA Bayle, 1878

Subgenus PROMYOPHORELLA Kobayashi and Tamura, 1955

Myophorella (Promyophorella) montanaensis (Meek)

Plate 3, figures $33-36,38-41$

Trigonia montanaensis Meek, 1873, U.S. Geol. Survey Terr. (Hayden), 6th Ann. Rept., 1872, p. 472.

Trigonia montanaensis Meek. White, 1880, U.S. Geol. Survey Terr. (Hayden), 12th Ann. Rept., 1878, pt. 1, p. 147, 148 , pl. 38 , fig. 2 a. 
Trigonia montanaensis Meek. Stanton, 1899, U.S. Geol. Survey Mon. 32, pt. 2, p. 619, 620.

?Trigonia ferrieri McLearn, 1924, Royal Soc. Canada Proc. and Trans., 3d ser., v. 18, sec. 4, p. 45, 46, pl. 5, figs. 2, 10.

The original description by Meek $(1873$, p. 472$)$ is as follows:

Of the type of $T$. signata Agassiz, but differing in its proportionally shorter form, with smaller nodiferous costae, while it has a row of nodes down the anterior lateral region of each valve, nearly as in T. navis Lamarck.

The species description by White $(1880$, p. 147,148$)$ is as follows:

Shell transversely elongate, somewhat arcuate and rudely subtetrahedral in marginal outline, gibbous anteriorly and narrowed and compressed behind; umbones elevated; from the umbo to the middle of the base the margin, as seen by side view, forms a continuous and almost true semicircular curve; from the middle of the base to the posterobasal angle the margin is slightly concave; posterior margin nearly straight or very slightly convex, forming, with the dorsal margin, a distinct slightly obtuse angle; dorsal margin concave, the appearance of concavity being increased by the elevation of the umbones; umbonal ridge distinct and prominent below, but becoming obsolete toward the beak. Surface in front of the umbonal ridge marked by ten or twelve curved varices, which start from the umbonal ridge and extend upward and forward. The first six or seven of these varices become broken up into distinct nodules a short space in front of the ridge. The forward ones of each varix or transverse row of nodules are larger than the others, and are arranged in a vertical row of strong nodules, which form a conspicuous feature of the shell. The corselet, a space behind and above the umbonal ridge, is marked only by numerous, distinct, nearly vertical, lines of growth.

The descriptions by Meek and White were based on the type figured by White and on one fragment consisting of the posterior three-fifths of a left valve. Both specimens are much corroded, and consequently, the ornamentation appears to be subdued as compared with other specimens of the species that are herein illustrated. In particular the umbonal ridge, or marginal carina, is distinct near the beak and bears prominent tubercles, and the postumbonal area bears a shallow median groove throughout its length and is bounded dorsally by a tuberculate carina. The escutcheon is about half as wide as the area, is depressed, and is nearly smooth but bears weak transverse riblets near the beak.

The specimen illustrated by White $(1880$, pl. 38 , fig. 2a) is herein designated as the lectotype of Myophorella (Promyophorella) montanaensis (Meek).

This species is very similar in shape and ornamentation to "Scaphogonia" argo Crickmay (1930, p. 51, 52 , pl. 5, figs. a, b) from middle Bajocian beds in British Columbia. In both species the anterior part of the disk bears strongly arcuate tuberculate ribs that diverge from the marginal carina at an acute angle and that meet the anterior margin at nearly a right angle. The tubercles on these ribs become stronger ventrally near the middle of the flank and then weaken slightly as the ribs curve anteriorly. Near the anterior margin the ribs weaken rather abruptly, or in some specimens are interrupted, but immediately anterior to the point of weakening or interruption, each rib bears a single prominent tubercle which is much stronger than the tubercles near the middle of the flanks. These prominent tubercles, as developed on successive ribs near the anterior margin, are almost in a row. They are generally followed anteriorly by from 1 to 3 weaker tubercles.

In both species the ribs on the posterior part of the disk have only a slight forward curvature near the ventral margin, are not interrupted or weakened near the ventral margin, and gradually become more strongly tuberculate ventrally.

The characteristics of the postumbonal area are likewise similar on the two species except that on "Scaphogonia" argo Crickmay the median groove is bounded ventrally by a weak tuberculate median carina, until the shell attains about $15 \mathrm{~mm}$ in length. Thereafter, both groove and carina disappear, according to Crickmay (1930, p. 52), and the middle of the area is marked only by a row of weak tubercles. In contrast, on "Trigonia" montanaensis Meek the median groove persists in the adult and is not bordered by tubercles or by a median carina.

Trigonia trafalgarensis Warren (1932, p. 18, pl. 3, figs. 5, 6) and T. ferrieri McLearn (1924, p. 45, pl. 5, figs. 2, 10) from the Fernie Formation of Alberta, Canada, are referred to Myophorella (Promyophorella) by Kobayashi and Tamura $(1955$, p. 93, 94). They are based on poorly preserved specimens that are difficult to compare with "Trigonia" montanaensis Meek but are described as being much less tuberculate. T. ferrieri McLearn, however, has the same shape and rib pattern as "T." montanaensis Meek. Furthermore, some specimens of the species obtained from the type locality on Grassy Mountain near Blairmore, Alberta, are as strongly tuberculate as "T." montanaensis Meek. It is doubtful, therefore, that $T$. ferrieri McLearn is a valid species.

Myophorella (Promyophorella) montanaensis Meek is common in the western interior region in beds of Bajocian to early Callovian age. The lectotype was obtained with the types of Trigonia americana Meek in beds of Bajocian age at the mouth of Yellowstone Canyon about $2 \frac{1}{2}$ miles south of Livingston, Mont.

The species is represented in the U.S. Geological Survey collections from the Carmel Formation of Utah by about 90 specimens.

Types: Lectotype USNM 131976; syntype USNM 7817; plesiotypes USNM 131977-131982. 
Occurrences in central and southern Utah: Carmel Formation at USGS Mesozoic locs. 964, 6281, 6282, 6941, 7341, 12555, 12557, $12560,12842,13532,16700,17269,17462,17492,20364,22170$, $24258,24259,25669,25673,25678,25680-25682,25687,26308$, $26310,27469,28472,28474$, and 28480 .

\section{Subgenus HAIDAIA Crickmay, 1930}

Myophorella (Haidaia) yellowstonensis Imlay, n. sp.

Plate 3, figures 27-32

The shell is subtrigonal in outline, a little longer than high. The anterior and ventral margins form a semicircle. The posterior margin is straight. The beak is small, curved backward, and located at $1 / 3-1 / 4$ of the length of the shell from the anterior end.

The disk is gently convex, ornamented with $16-20$ finely crenulated arcuate ribs that diverge acutely from the marginal carina. These ribs are narrow, rounded, and are steepest on their umbonal side. The crenules are very fine and are most distinct on the posteroventral side of the ribs. The marginal carina is narrow, distinct curved, and tuberculate. The area is about one-third as wide as the disk, is divided medially by a groove, is bounded dorsally by a tuberculate carina that is broader than the marginal carina, and is ornamented by fine transverse riblets. These riblets are fairly widely spaced near the beak but become closely spaced posteriorly. Most of them terminate in the tubercles on the marginal and inner carinae, but some terminate between the tubercles. The escutcheon is depressed, is about half as wide as the area, and is crossed by fine widely spaced riblets. The holotype is $21 \mathrm{~mm}$ long and $15 \mathrm{~mm}$ high.

The species shows some variation in the strength of the tubercles on the carinae and in the density of the riblets on the area.

It differs from Myophorella (Haidaia) dawsoni (Whiteaves) (1884, p. 231, pl. 31, figs. 1, 1a) from western British Columbia by its much smaller size, more trigonal shape, finer ornamentation, and the lack of a row of tubercles in the middle of the area. It shows much more resemblance to $M$. (H.) crenulata Kobayashi and Tamura (1955, p. 100, pl. 5, figs. 8, 9a, b, 10) from the Upper Jurassic of Japan, but its carinae are tuberculate instead of crenulate, its area is less depressed, and the riblets on the area are more widely spaced near the beak.

This species is moderately common in western Montana, western Wyoming, southeastern Idaho, and northern Utah in beds of Bajocian age. In contrast, only one specimen has been found in Utah south of the Uinta and Wasatch Mountains.

Types: Holotype USNM 131983; paratypes USNM 131984131988.

Occurrence in central Utah: Carmel Formation at USGS
Mesozoic loc. 13531. The holotype and one of the paratypes are from Mesozoic loc. 27624 in Montana. The other paratypes are from Mesozoic loc. 21385 in Montana, Mesozoic loc. 20967 in Wyoming, and Mesozoic loc. 13531 in Utah.

\section{Genus ASTARTE Sowerby, 1816}

Subgenus COELASTARTE Boehm, 1893

Astarte (Coelastarte) livingstonensis Imlay, n. sp.

Plate 4, figures 1-4

The shell is very flat, elongate, subrectangular in outline, inequilateral. Posterior dorsal margin long, straight, gently inclined. Posterior margin straight, nearly vertical, rather short, meeting the dorsal margin sharply at a subobtuse angle, meeting the ventral margin rather abruptly at nearly a right angle. Ventral margin slightly convex on immature specimens but becoming nearly straight on adults. Anterior margin short, rounding evenly into ventral margin. Anterior dorsal margin slightly concave, shorter and steeper than the posterior dorsal margin, meeting the anterior margin at nearly a right angle. Beaks small, compressed, rising moderately above the hinge line, situated from $1 / 3$ to $2 / 5$ of the length of the shell from the anterior end. A low oblique ridge trends from the beak to the posteroventral angle.

The ornamentation consists mainly of narrowly rounded concentric ribs that follow the outline of the shell, bend sharply where they cross the oblique ridge, meet the posterior dorsal margin at nearly a right angle, and number from 17 to 20 on the largest specimens. The interspaces are broadly rounded and are wider than the ribs. On the largest adult the ribs tend to weaken near the posterior margin. Nearly microscopic growth lines cover the entire surface. The holotype has a length of $23 \mathrm{~mm}$ and a height of $12.5 \mathrm{~mm}$.

This species is distinguished from most described species of Coelastarte by having a more salient beak farther from the anterior end of the shell and by a shorter posterior margin. One of the most similar is $A$. (C.) rectangula Arkell (1934, p. 248, pl. 33, fig. 16), from beds of late Oxfordian age in England, but that species evidently has a much longer posterior margin, a less salient beak, and a weakly concave ventral margin. $A$. (C.) aytonensis Lycett (not Bean) (Lycett, 1863, pl. 40, fig. 13; Arkell, 1934, p. 246) from the Great Oolite of England is likewise similar to $A$. (C.) lvvingstonensis Imlay, n. sp., but has a broader anterior margin, a longer posterior margin, and its beak is nearer the anterior end of the shell.

This species has been obtained in collections from the western part of the western interior region in beds of Bajocian age but is somewhat less common than other species of Astarte found in those beds. In the Geological 
Survey collections from southern Utah, it is represented by one specimen.

Types: Holotype USNM 131989; paratypes USNM 12220, 131990, 131991.

Occurrences in Utah: USGS Mesozoic locs. 12558, 17269. The species is probably represented also at Mesozoic locs. 11438, 28467, 28472.

Occurrences of type specimens: The large paratype shown on pl. 1, fig. 2, is in a collection made by A. C. Peale on the west side of the Lower Canyon of the Yellowstone River near Livingston, Mont. The other paratypes are from Mesozoic locs. 21385 and 25866. The holotype is from Mesozoic loc. 25866. All these localities are in southwestern Montana.

\section{Astarte (Coelastarte) n. sp. undet.}

Plate 4, figures 6-8

This species is represented in central and southern Utah by about 25 molds. It differs from $A$. livingstonensis Imlay, $\mathrm{n}$. sp. by having a higher rounder less elongate shell, by its beaks being nearer the anterior end of the shell, and by its ribbing fading toward the margins.

Figured specimens: USNM 131993, 132011, 132012.

Occurrences in central and southern Utah: USGS Mesozoic locs. 17269, 27474, 28461, 28494, and 28495.

Astarte (Coelastarte) cf. A. (C.) interlineata (Lycett)

Plate 4, figure 5

One specimen of Coelastarte from southern Utah is comparable in shape and sparseness of ribbing to A. (C.) interlineata (Lycett) (Morris and Lycett, 1853, p. 87, pl. 9, figs. 14, 15a, b) from the Middle Jurassic of England. It appears to have a less rectangular outline and a slightly convex instead of a straight ventral margin.

Figured specimen: USNM 131992.

Occurrence in southern Utah: USGS Mesozoic loc. 17269.

\section{Genus PRONOELLA Fischer 1887}

Pronoella cinnabarensis (Stanton)

Plate 4, figures 27-30

Cyprina? cinnabarensis Stanton, 1899, U.S. Geol. Survey Mon. 32 , pt. 2, p. 621,622 , pl. 72, figs. 7,8 .

The original description is as follows:

Shell of medium size, moderately convex, subcircular in outline, with prominent submedian beaks; dorsal margin excavated in front of the beaks, gently sloping behind, and in both cases passing gradually into the rounded ends; posterior end in some individuals slightly straightened, so as to become almost yertically subtruncate; ventral margin broadly rounded; surface sculpture unknown. There is a very obscure posterior umbonal ridge, and the muscular and pallial impressions are not clearly shown on the cast.

One cast showing impression of part of hinge has three strong cardinal teeth, of which the posterior one is very long and oblique.
The specimen is not in condition to show whether lateral teeth are present.

Height of an average shell, $28 \mathrm{~mm}$; length $32 \mathrm{~mm}$; convexity of two valves united $15 \mathrm{~mm}$. The largest specimens in the collection the have corresponding dimensions about one-fifth greater.

The hinge described by Stanton is on a right valve and is illustrated herein. It bears an elongate posterior lateral tooth that is followed by a deep, fairly narrow pit. Beyond is a triangular shaped bifid posterior cardinal tooth. This is followed by a wide triangular-shaped pit beyond which is a moderately prominent tooth that occurs at the posterior end of a thin elongate anterior lateral tooth.

The presence of a bifid posterior cardinal tooth indicates that this species belongs to Pronoella rather than to Eocallista or Isocyprina (Cox, 1947, p. 144, 145). The presence of a faint umbonal ridge also favors an assignment to Pronoella.

The specimen illustrated by Stanton is herein selected as lectotype of $P$. cinnabarensis (Stanton). This is from the ridge southwest of the second crossing of the Snake River, Yellowstone National Park. The specimen on which the hinge is exposed is from the ridge between Red and Basin creeks near Sheridan Peak.

Internal molds having the shape of $P$. cinnabarensis (Stanton) are fairly common in beds of Bajocian to early Callovian age in the western part of the western interior region and particularly near Yellowstone National Park. Lack of distinctive markings and poor preservation generally make specific identification difficult or uncertain. The Geological Survey collections from central and southern Utah contain about 40 internal molds comparable with the species. Such occur at USGS Mesozoic localities 6284, 6941, 12556, 12835, 12839 , 16624, 17462, 18076, 20351, 20358, 20359, $25671,25686,27470$, and 28493.

Types: Lectotype USNM 28939; syntype USNM 30648.

\section{Pronoella uintahensis Imlay, n. sp.}

Plate 4, figures $15-23$

The shell is subovate, wider than high, moderately inflated. The umbones are broadly rounded, curved inward and forward, and are situated at $1 / 4-1 / 5$ of the length of the shell from the anterior end. The posterodorsal margin is gently convex, gently inclined, and meets the posterior margin at an obtuse angle. The anterodorsal margin is slightly excavated. The anterior margin is rather sharply convex. The ventral margin is broadly convex, rounds evenly into the anterior margin and sharply into the posterior margin. The posterior margin is high and steeply inclined. The lunule is poorly defined. The escutcheon is long, narrow, deep and is bounded by a ridge. A weak 
ridge curves downward from the umbo to the posteroventral corner. The shell surface is nearly smooth, but toward the margins it bears very weak growth lines and a few larger growth imbrications.

The hinge line is fairly well exposed on two silicified specimens from the Uinta Mountains (USGS Mesozoic loc. 20536). The hinge on the right valve bears an elongate posterior lateral tooth. The posterior cardinal tooth is triangular, thick, and bifid. It is separated by a deep pit from a less prominent median(?) cardinal tooth that occurs at the posterior end of a thin oblique lateral tooth. The hinge on the left valve bears a thick prominent median cardinal tooth. This is separated by a deep pit from a thin posterior cardinal tooth and by a smaller shallower pit from a moderately thin anterior cardinal tooth that bends abruptly into an anterior lateral tooth.

The paratype shown on plate 4 , figures $20-22$, has a height of $23 \mathrm{~mm}$, a length of $26 \mathrm{~mm}$, and a convexity (both valves) of $11.5 \mathrm{~mm}$.

This species is distinguished from Cyprina? iddingsi Stanton (1899, p. 622, pl. 73, fig. 9) (pl. 4, figs. 24, 25) by having more prominent umbones that are nearer the anterior end and by its posterior margin being higher. Pronoella cinnabarensis (Stanton) (1899, p. 621, pl. 73, figs 7,8$)$ has a fainter umbonal ridge, and its umbones are nearer the middle of the shell. Among foreign species it shows considerable resemblance to Pronoella ravenscarensis Cox (1947, p. 175, 176, text figs. 56a, b, on p. 181, pl. 10, fig. 86) from the Middle Jurassic of England but has more prominent umbones, a higher posterior margin, and a weak postumbonal ridge.

This species is represented in the Geological Survey collections from southern and central Utah by 140 specimens. Most of these are internal molds preserved in a limestone matrix, but about a dozen specimens from one locality (USGS Mesozoic loc. 25685) are silicified, and these show the external shape of the species very well. In addition, the species may be represented by another 65 specimens that are very poorly preserved.

The specimens showing the hinge features are from Uintah County, northeast Utah, and were collected from the base of the Carmel Formation in Steinaker Draw, near Vernal (USGS Mesozoic loc. 20536) in sec. 26, T. 3 S., R. 21 E.

This species is assigned to Pronoella rather than Eocallista because of the presence of a weak postumbonal ridge and of a bifid posterior cardinal tooth in the right valve. This bifid tooth is present on several right valves from USGS Mesozoic locality 20536 in northeast Utah. The hinge is not exposed on any of the specimens from central and southern Utah. Consequently the identification of $P$. uintahensis Imlay, n. sp., in those parts of Utah is based solely on external shape.

Types: Holotype, USNM 131994; paratypes, USNM 131995131999.

Occurrences in central and southern Utah: USGS Mesozoic locs. $963,964,7341,9113,10116,11439,11445,12581,12840,16624$, $16700,16701,17054,14712,17495,19422,21446,20352,25670$, 25685, 27469, 27474, 28469, 28470, 28491, 28494, and 28595. Poorly preserved specimens comparable to Pronoella uintahensis Imlay, n. sp., occur at Mesozoic locs. 5263, 15498, 16702, 16703, $17018,17030,17351,17494,18672,24260,25683,25826,26309$, 27475 , and 28462 .

\section{Genus LUCINA Bruguière, 1797}

Lucina sp.

The genus is represented in southern Utah by four poorly preserved specimens. These are orbicular in shape, bear fine concentric growth lines, and have a weak furrow extending backward from the umbo. The genus is rare in the Jurassic of the western interior region.

Occurrences in southern Utah: USGS Mesozoic locs. 963 and 25671.

\section{Genus QUENSTEDTIA Morris and Lycett, 1854}

Quenstedtia aff. Q. bathonica (Morris and Lycett)

Plate 4, figures 9-11

Three specimens from southern Utah resemble Quenstedtia bathonica (Morris and Lycett) (1854, p. 95, pl. 13, fig. 14; Cox, 1929, pl. 13, fig. 10) in shape and in the position of their umbones but differ by having a more distinct ridge extending from the umbo to the posteroventral angle and a less rounded posterior end.

Figured specimens: USNM 132000-132002.

Occurrences: USGS Mesozoic locs. 12835, 16701, and 17494.

\section{Genus PLEUROMYA Agassiz, 1843}

\section{Pleuromya subcompressa (Meek)}

Myacites (Pleuromya) subcompressa Meek, 1873, U.S. Geol. Survey Terr. (Hayden) 6th Ann. Rept., 1872, p. 472.

Myacites (Pleuromya) subcompressa Meek, 1877, U.S. Geol. Explor. 40th. Parallel (King), v. 4, pt. 1, p. 136, pl. 12, figs. $6,6 a$.

Myacites (Pleuromya) subcompressa Meek. White, 1880, U.S. Geol. Survey Terr. (Hayden) 12th Ann. Rept., 1878, pt. 1, p. 151, pl. 38, figs. 5a-e.

Pleuromya subcompressa (Meek). Stanton, 1899, U.S. Geol. Survey Mon. 32, pt. 2, p. 627, pl. 74, figs. 8-11.

Pleuromya subcompressa (Meek). Crickmay, 1936, Geol. Soc. America Bull., v. 47 , p. 556 , pl. 1, fig. 6, pl. 2, figs. 5, 12, pl. 3, fig. 10 .

Both White and Stanton emphasized the high variability of this species in form and sculpture and illustrated a number of specimens to show the range in variation. In particular Stanton (1899, p. 626) noted 
that "Extreme variations approach the plicate Pleuromya weberensis Meek on the one hand and the nearly smooth Pleuromya newtoni Whitfield on the others."

Of these species, Pleuromya newtoni Whitfield (1880, p. 367,368 , pl. 5 , figs. 19,20 ) has much finer ribbing, is more elongate, has lower umbones, and occurs at a higher stratigraphic level in beds of late Oxfordian age in the Black Hills (Imlay, 1947, p. 262). The type of P. weberensis (Meek) (1877, p. 137, pl. 12, figs. 11, 11a) is from Weber Canyon, Utah, where it is associated with $P$. subcompressa (Meek) (1877, p. 136). It lacks the posterior end of its shell and is possibly only a finely ribbed variant of $P$. subcompressa (Meek).

Among Canadian species, the most similar is $P$. postculminata McLearn (1924, p. 55, pl. 9, fig. 6). This species is apparently differentiated by having a shallow sulcus that extends from the umbo to near the middle of the ventral margin where it produces an emargination. On P. subcompressa (Meek) an emargination of the ventral margin is generally less distinct and occurs nearer the anterior end.

$P$. subcompressa (Meek) is very common in beds of Bajocian to Callovian age in central and western Wyoming and Montana, in southeastern Idaho, and in northern Utah. The Geological Survey collections from central and southern Utah contain only six specimens of this species.

The original description of P. subcompressa (Meek) is in a footnote to a list headed "Near Lower Canyon of Yellowstone River." Consequently the primary types should include the specimens from the Lower Canyon of the Yellowstone River that Meek studied (see Crickmay, 1936, p. 557). These specimens were collected by A. C. Peale in 1872 and were illustrated in part by C. A. White in 1880 (pl. 38, figs. $5 \mathrm{a}-\mathrm{d}$ ). The original collection made by A. C. Peale includes seven specimens, all technically cotypes. Of these the writer selects as lectotype the specimen illustrated by White on his plate 38 , figures $5 \mathrm{c}$, d.

Types: Cotypes USNM 8061; plesiotypes USNM 8180, 28958-28961. The specimen from Weber Canyon, Utah, figured by Meek (1877, pl. 12, figs. 6, 6a) is lost.

Occurrences in central and southern Utah: USGS Mesozoic locs. 17462, 18922, 20351, 20364, and 25682.

Genus PHOLADOMYA G. B. Sowerby, 1823

\section{Pholadomya kingi Meek}

Plate 4, figure 36

Pholadomya kingi Meek, 1873, U.S. Geol. Survey Terr. (Hayden) 6th Ann. Rept., 1872, p. 473.

Pholadomya kingi Meek. White, 1880, U.S. Geol. Survey Terr. (Hayden) 12th Ann. Rept., 1878, pt. 1, p. 150, pl. 38, figs. $3 \mathrm{a}, \mathrm{b}$.
Pholadomya kingi Meek. Stanton, 1899, U.S. Geol. Survey Mon. 32, pt. 2, p. 624, pl. 74, figs. 1-3.

Pholadomya kingi Meek. Butler and others, 1920, U.S. Geol. Survey Prof. Paper 111, pl. 10, figs. 1, 2.

This species is characterized by having from 8 to 14 strong radial ribs that cover the middle part and most of the anterior part of the shell. It is moderately common in the western part of the western interior region in beds of Bajocian to early Callovian age. Four specimens have been found in central Utah.

Types: Holotype USNM 7815; plesiotypes USNM 28957, 132003.

Occurrences in central Utah: USGS Mesozoic locs. 6941, 12558, and 17269 .

\section{Pholadomya inaequiplicata Stanton}

Plate 4, figures 37,38

Pholadomya inaequiplicata Stanton, 1899, U.S. Geol. Survey Mon. 32, pt. 2, p. 625, pl. 74, fig. 4 .

This species, as characterized by Stanton, differs from $P$. kingi Meek by being smaller, more ventricose, less elongate, and by having about 20 radiating ribs that cover more of the shell. Judging by the collections now available, $P$. inaequiplicata Stanton shows the same variation in shape as $P$. kingi Meek and differs only by having finer more numerous ribs. Both species have the same vertical range and are commonly associated.

Pholadomya inaequiplicata Stanton is represented by 27 specimens in collections from central and southern Utah.

Types: Holotype USNM 28956; paratypes USNM 30668; plesiotypes USNM 132004, 132005.

Occurrences in central and southern Utah: USGS Mesozoic locs. 6941, 7890, 25671, 25681, 26307, 28468, and 28493.

\section{Genus GONIOMYA Agassiz, 1842}

\section{Goniomya montanaensis Meek}

Plate 4, figures 34,35

Goniomya montanaensis Meek, 1873, U.S. Geol. Survey Terr. (Hayden) 6th Ann. Rept., 1872, p. 473.

Goniomya montanaensis Meek. White, 1880, U.S. Geol. Survey Terr. (Hayden) 12th Ann. Rept., 1878, p. 151, pl. 37, fig. 8 a.

In this species the ribs do not form a sharp $V$ near the middle of the flanks as is common in Goniomya, but rather resemble a $V$ whose apex is spread apart and connected by a horizontal bar. The anterior part of the shell bears strong ribs that are steeply inclined, the middle part of the shell bears weak horizontal ribs, and the posterior part of the shell bears strong vertical ribs. The junction of these ribs is rather abrupt on the upper part of the shell but becomes less abrupt near the ventral margin where all the ribs become weaker. 
This species has a rib pattern similar to that on Goniomya hemicostata Morris and Lycett (1853, p. 120, pl. 12, fig. 3) from the Great Oolite of England, but its beaks are situated more anteriorly, and its anterior margin is more broadly rounded.

Goniomya montanaensis Meek is rare in beds of Bajocian age in the western interior region. The holotype was obtained from the middle of the Piper Formation on the west side of the Lower Canyon of the Yellowstone River near Livingston, Mont. A single external mold of the species occurs in the Geological Survey collections from central Utah.

Type: Holotype USNM 7814.

Occurrence in central Utah: USGS Mesozoic loc. 20351.

\section{Goniomya? n. sp. undet. \\ Plate 4, figure 39}

This species is represented by a single highly convex left valve on which the umbo is situated about one-third of the length of the shell from the anterior end. The dorsal part of the valve bears strong ribs that are bent sharply twice to form two acute $V$-shaped folds of which the most anterior is the larger. Some of the ribs on the posterior part of the larger fold continue beyond the apex of the $V$ as far as the anterior ventral margin as fairly strong ribs. The remainder of the ventral margin and all of the posterior margin are smooth except for growth lines. In addition, the anterior part of the shell anterior to the largest $V$ bears 8 or 9 weak ribs directed obliquely downward and anteriorly at nearly a right angle to the axis of the $V$.

The zigzag rib pattern bears some resemblance to that on $G$. normanniana (d'Orbigny) as figured by Arkell $(1935$, p. 48 , figs. 8,9$)$, but the large $V$-shaped fold as well as the umbo is much nearer the anterior end of the shell, and the apex of the $V$ is not separated by a horizontal bar. The generic status of the species is questionable because of the unusual direction of the ribs at the anterior end of the shell.

Figured specimen: USNM 132006.

Occurrence in Utah: USGS Mesozoic loc. 6944.

\section{Genus MYOPHOLAS H. Douville, 1907 \\ Myopholas hardyi Imlay, n. sp. \\ Plate 4, figures 12-14}

The genus Myopholas is represented by two fairly complete external molds and by several incomplete molds in the Arapien Shale of central Utah. These show that the shell is highly convex, elongate ovate, and nearly twice as long as high. The posterior end is more narrowly rounded than the anterior end and apparently is gaping. The umbones are broad, strongly incurved, and slightly anterior to the middle of the shell. The anterior part of the shell bears five strong sharp widely spaced radial ribs. The medial part of the shell bears 6 or 7 less sharp closely spaced ribs. These ribs are bound posteriorly by a deep and broad sulcus that extends radially from the posterior side of the umbo to the ventral margin. The sulcus shows faint traces of radial ribbing, but the posterior end of the shell does not bear radial ribs. The entire surface of the shell is marked by weak concentric ribs.

The holotype has a length of $11.5 \mathrm{~mm}$, a height of $6 \mathrm{~mm}$, and a convexity (one valve) of about $2.5 \mathrm{~mm}$.

The Utah specimens of Myopholas are similar in appearance to $M$. acuticosta (J. de C. Sowerby) (1827, p. 88, pl. 546, fig. 1; Arkell, 1935, p. 349, pl. 45, figs. 14-16; Lissajous, 1923, pl. 32, figs. 8, 8a) from the Bathonian and Callovian of Europe but have a broader deeper radial sulcus and lack radial ribs posterior to the sulcus.

Types: Holotype USNM 132007; paratype USNM 132008.

Occurrences in central Utah: USGS Mesozoic locs. 21448 and 21449.

This species is named after C. T. Hardy, Utah State Agricultural College, who collected the type specimens.

\section{Genus CERCOMYA Agassiz, 1843}

Cercomya punctata (Stanton)

Plate 4, fig ure 31

Anatina (Cercomya) punctata Stanton, 1899, U.S. Geol. Survey Mon. 32 , pt. 2, p. 628, pl. 74, fig. 5 .

Anatina cf. A. punctata Stanton. MeLearn, 1924, Royal Soc. Canada, Proc. and Trans., 3d ser., v. 18, sec. 4 , p. 56, pl. 9 , fig. 11.

?Anatina (Cercomya) semiradiata Whiteaves, 1900, Canada Geol. Survey, Mesozoic fossils, v. 1, pt. 4, p. 288, pl. 37, fig. 4 .

This species is distinguished from most species of Cercomya by being less elongate and by the presence of distinct granular radiating ribs on the middle of the posterior part of the shell. The specimen of Cercomya from the Callovian beds of Alberta (McLearn, 1924, p. 56 , pl. 9, fig. 11) is nearly identical in appearance with the holotype of Stanton's species. Cercomya semiradiata Whiteaves (1900, p. 288, pl. 37, fig. 4) from the Callovian beds of the Queen Charlotte Islands compares closely with Stanton's species in shape and in the presence of fine radial ribs but may have a more swollen triangular area posterior to the narrow median sulcus that descends from the beak to the ventral margin. Among European species probably the most similar is C. striata Agassiz (1842, p. 149, pl. 11, figs. 13-15; Loriol, 1872 , p. 197, pl. 12, figs. 10, 11; Cottreau, 1928 , p. 95,1929 , pl. 52, fig. 14), but it is definitely much more elongate posteriorly, and the radial striae are not nearly as strong as the radial ribs on $C$. punctata (Stanton).

Cercomya punctata (Stanton) is moderately common in the western part of the western interior region in 
beds of Bajocian to early Callovian age. It is represented by three specimens from Garfield County, Utah, in the Geological Survey collections.

Types: Holotype USNM 28945; paratype USNM 30593 plesiotype USNM 132009.

Occurrences in southern Utah: USGS Mesozoic locs. 25671 and 26307.

\section{Genus THRACIA Leach, 1823}

Thracia weedi Stanton

Plate 4, figures 32,33

Thracia weedi Stanton, 1899, U.S. Geol. Survey Mon. 32, pt. 2, p. 627 , pl. 75 , figs. $1-3$.

The original description is as follows:

Shell of medium size, thin, compressed, elongate, subelliptical in outline; beaks rather prominent, submedium; dorsal margin sloping rather rapidly and almost equally before and behind the beaks; anterior end broadly rounded, most prominent below; posterior end subtruncate; ventral margin slightly convex, somewhat sinuous toward the posterior end; posterior umbonal ridge narrow and sharply defined; surface marked by irregular concentric undulations and by numerous growth lines.

The preceding description needs some modification. The beaks are actually slightly posterior to the median part of the shell and are directed slightly backward, the posterior dorsal margin is slightly concave, and on most specimens the right valve is distinctly larger than the left. The shape varies considerably from one specimen to another, but this is a characteristic of the genus (Arkell, 1936, p. 355).

This species differs from Thracia convexa Warren (1932, p. 26, pl. 1, fig. 19) from beds of Bajocian age near Blairmore, Alberia, by being much less convex and by its beaks being nearer the median part of the shell. Its umbones are much less prominent than in $T$. semiplanata Whiteaves $(1884$, p. 221,222 , pl. 29 , figs. 5, 5a-c) from beds of Callovian age on the Queen Charlotte Islands. Among European species it shows more resemblance to $T$. lens Agassiz (1845, p. 267, pl. 36, figs. 1-15), but its beaks are smaller and more centrally situated and it is apparently more compressed.

Thracia weedi Stanton is fairly common in shaly beds of Bajocian age in and near Yellowstone National Park. In southern Utah it is represented by 64 specimens of which 60 are from a single spot in Garfield County.

The specimen illustrated by Stanton (1899) on his plate 75 , figure 2 , is herein selected as the lectotype of Thracia weedi Stanton. This specimen is from USGS Mesozoic locality 1145 from a streambed west of Little Quadrant Mountain in Yellowstone National Park.

Types: Lectotype USNM 28994; syntypes USNM 28943; plesio type USNM 132010.

Occurrences in southern Utah: USGS Mesozoic locs. 16701, 16703, 25671, 26307, 28468, 28494, and 28495.

\section{LITERATURE CITED}

Agassiz, Louis, 1840-45, Études critiques sur les mollusques fossiles: Neuchatel, 3 v. (v. 1. (1840) Les Trigonies du Jura et de la Craie Suisses; v. 2-3 (1842-45) Monographie des Myes).

Arkell, W. J., 1929-37, A monograph of British corallian Lamellibranchia: London, Palaeontographical Soc., 392 p., $56 \mathrm{pls}$.

Baker, A. A., 1947, Stratigraphy of the Wasatch Mountains in the vicinity of Provo, Utah: U.S. Geol. Survey Oil and Gas Inv. Prelim. Chart 30.

Baker, A. A., Dane, C. H., and Reeside, J. B., Jr., 1936, Correlation of the Jurassic formations of parts of Utah, Arizona, New Mexico and Colorado: U.S. Geol. Survey Prof. Paper 183,66 p., 33 pls., 16 figs.

Butler, B. S., and others, 1920, The ore deposits of Utah: U.S. Geol. Survey Prof. Paper 111, 672 p., 57 pls., 74 figs.

Cottreau, Jean, 1928-29, Types du prodrome de paléontologie stratigraphique universelle d'Orbigny, v. 2, Callovien-Portlandien: Annales paléontologie, v. 17 (1928), p. 65-96, pls. $47-51$; v. 18 (1929), p. $97-132$, pls. $52-57$.

Cox, L. R., 1929, Notes on the Mesozoic family Tancrediidae, with descriptions of several British Upper Jurassic species, and of a new genus, Eodonax; Annals and Mag. Nat. History, ser. 10 , v. 3 , p. $569-594$, pls. $13,14$.

1947, The lamellibranch family Cyprinidae in the Lower Oolites of England: Malacological Soc. London Proc., v. 47, pt. 4 , p. 141-184, pls. 8-10, 56 text figs.

1952, The Jurassic lamellibranch fauna of Cutch (Kachh). No. 3, Families Pectinidae, Amusiidae, Plicatulidae, Limidae, Ostreidae and Trigoniidae (supplement): India Geol. Survey Mem., Palaeontologia Indica, ser. 9, v. 3, pt. 4, 128 p., $12 \mathrm{pls}$.

Crickmay, C. H., 1930, The Jurassic rocks of Ashcroft, British Columbia: California Univ., Dept. Geol. Sci., Bull., v. 19 , no. 2 , p. $23-74$, pls. $2-7$.

- 1936, Study in the Jurassic of Wyoming: Geol. Soc. America Bull., v. 47, p. 541-564, pls. 1-3.

Gilluly, James, 1929, Geology and oil and gas prospects of part of the San Rafael Swell, Utah: U.S. Geol. Survey Bull. 806-C, 128 p., pls. 30-35.

Gottsche, Carl, 1878, Ueber jurassische Versteinerungen aus der argentinischen Cordillera: Palaeontographica, Supp. 3, no. 2, pt. 3, p. 1-51, pls. 1-8.

Gregory, H. E., 1950, Geology and geography of the Zion Park region, Utah and Arizona: U.S. Geol. Survey Prof. Paper 220,200 p., 5 pls., 133 figs.

1951, The geology and geography of the Paunsaugunt region, Utah: U.S. Geol. Survey Prof. Paper 226, 116 p., 5 pls., 63 figs.

Hall, James, and Whitfield, R. P., 1877, Paleontology: U.S. Geol. Explor. 40th Parallel (King), v. 4, pt. 2, p. 199-302, pls. $1-7$.

Hardy, C. T., 1952a, Eastern Sevier Valley, Sevier and San Pete Counties, Utah-with reference to formations of Jurassic age: Utah Geol. and Mineralog. Survey Bull. 43, 98 p. 1952b, Stratigraphy and structure of the Arapien shale and Twist Gulch formation in Sevier Valley, Utah: Abstracts of Doctoral Dissertations, no. 62, Ohio State Univ. Press, p. 91-97.

- 1962, Mesozoic and Cenozoic stratigraphy of northcentral Utah: Brigham Young Univ. Geology Studies, v. 9, pt. 1 , p. $50-64$.

Hardy, C. T., and Zeller, H. D., 1953, Geology of the westcentral part of the Gunnison Plateau, Utah: Geol. Soc. America Bull., v. 64, p. 1261-1278. 
Imlay, R. W., 1947, Marine Jurassic of Black Hills area, South Dakota and Wyoming: Am. Assoc. Petroleum Geologists Bull., v. 31 , no. 2, p. 227-273, 3 figs.

1950, Jurassic rocks in the mountains along the west side of the Green River Basin, in Wyoming Geol. Assoc., Southwest Wyoming, Guidebook 5th Ann. Field Conf.: Casper, Wyo., p. 37-48, 3 figs.

1952a, Marine origin of the Preuss sandstone of Idaho, Wyoming, and Utah: Am. Assoc. Petroleum Geologists Bull., v. 36 , no. 6, p. 1735-1753, 4 figs.

1952b, Correlation of the Jurassic formations of North America exclusive of Canada: Geol. Soc. America Bull., v. 63 , p. $953-992,2$ pls., 4 figs.

1953, Characteristics of the Jurassic Twin Creek linestone in Idaho, Wyoming and Utah, in Intermountain Association of Petroleum Geologists, Guide to the geology of northern Utah and southeastern Idaho, 4th annual field conference 1953: Salt Lake City, Utah, p. 54-62.

- 1956a, Interpretations of the marine Jurassic fossil record at Lower Slide Lake, Teton County, Wyoming, in Wyoming Geol. Assoc., Jackson Hole, Guidebook, 11th Ann. Field Conf.: Casper, Wyo., p. 70-71.

1956b, Marine Jurassic exposed in Bighorn Basin, Pryor Mountains, and northern Bighorn Mountains, Wyoming and Montana: Am. Assoc. Petroleum Geologists Bull., v. 40, no. 4, p. 562-599, 7 figs.

1957, Paleoecology of Jurassic seas in the western interior of the United States: Geol. Soc. America Bull., v. 67, p. 469-504, 8 figs.

Johnson, K. D., 1959, Structure and stratigraphy of the Mount Nebo-Salt Creek area: Brigham Young Univ. Research Studies, Geol. Ser., v. 6, no. 6, 49 p.

Kobayashi, Teiichi, and Mori, Kazuo, 1955, The Vaugoniinae from the Kitakami Mountains in north Japan; On the Jurassic trigonians in Japan, part III: Japanese Jour. Geology and Geography, v. 26, nos. $1-2$, p. $73-88$, pls. 3,4 .

Kobayashi, Teiichi, and Tamura, Minoru, 1955, The Myophorellinae from north Japan; Studies on the Jurassic trigonians in Japan, part IV: Japanese Jour. Geology and Geography, v. 26, nos. 1-2, p. 89-103, pls. 5, 6.

Lissajous, Marcel, 1923, Étude sur la faune du Bathonien des environs de Macon: Lyon Univ., Fac. Sci. Lab. géologie, Travaux, Mem. 3, 286 p., 33 pls.

Logan, W. N., 1900, The stratigraphy and invertebrate faunas of the Jurassic formation in the Freeze-out Hills of Wyoming: Kansas Univ. Quart., v. 9, no. 2, ser. A, p. 109-134, pls. 25-31.

Loriol, P. de, 1872, Description des fossiles, in P. de Loriol, Royer, E., and Tombeck, H., Monographie paléontologique et géologique des étages supérieurs de la formation jurassique du departement de la Haute-Marne: Soc. linnéenne Normandie, v. 16, p. 1-484, pls. 1-26.

Lycett, John, 1863, A supplementary monograph on the Mollusca from the Stonesfield Slate, Great Oolite, Forest Marble, and Cornbrash: London, Palaeontographical Soc., 129 p., pls. 31-45.

- 1872-1883, A monograph of the British fossil Trigoniae: London, Palaeontographical Soc., $245+19$ p., $41+4$ pls.

McLearn, F. H., 1924, New pelecypods from the Fernie formation of the Alberta Jurassic: Royal Soc. Canada Proc. and Trans., 3 d ser., v. 18 , sec. 4 , p. 39-61, 9 pls.

1926, New Jurassic species from the Hazelton Group of British Columbia: Canada Geol. Survey Bull. 44, Geol. Ser. no. 46, p. $89-99$, pls. $19-25$.
Mansfield, G. R., 1927, Geography, geology, and mineral resources of southeastern Idaho: U.S. Geol. Survey Prof. Paper 152, 453 p., 70 pls., 46 figs.

Meek, F. B., 1860, Descriptions of new fossil remains collected in Nebraska and Utah, by the exploring expeditions under the command of Capt. J. H. Simpson ***: Acad. Nat. Sci. Philadelphia Proc., 1860, p. 308-315.

- 1873, Preliminary paleontological report, consisting of lists and descriptions of fossils, with remarks on the ages of the rocks in which they were found, etc., etc.: U.S. Geol. Survey Terr. (Hayden) 6th Ann. Rept., 1872, p. 431-518. - 1876, Report on the paleontological collections of the expedition, in Simpson, J. H., Report of explorations across the Great Basin of the Territory of Utah $* * *$ in 1859: Washington, D.C., p. 337-373.

1877, Paleontology: U.S. Geol. Explor. 40th Parallel (King), v. 4 pt. 1, p. 1-197, pls. 1-17.

Meek, F. B., and Hayden, F.V. 1858, Descriptions of new organic remains collected in Nebraska Territory $* * *$ : Acad. Nat. Sci. Philadelphia Proc. 1858 , p. 41-59.

- 1860, Descriptions of new organic remains from the Tertiary, Cretaceous and Jurassic rocks of Nebraska: Acad. Nat. Sci. Philadelphia Proc. 1860, p. 175-185.

1865, Palaeontology of the upper Missouri; invertebrates: Smithsonian Inst., Smithsonian Contr. Knowledge, v. 14, no. 172 , p. 1-135, pls. 1-5.

Morris, John, and Lycett, John, 1850-54, A monograph of the Mollusca from the Great Oolite, chiefly from Minchinhampton and the coast of Yorkshire: London, Palaeontographical Soc., 3 v. in 1 (Pt. 1, Univalves; Pt. 2-3, Bivalves).

Pipiringos, G. N., 1957, Stratigraphy of the Sundance, Nugget and Jelm formations in the Laramie basin, Wyoming: Wyoming Geol. Survey Bull. 47, 63 p., 5 pls. 3 figs.

Schultz, A. R., 1914, Geology and geography of a portion of Lincoln County, Wyo.: U.S. Geol. Survey Bull. 543, 141 p., $11 \mathrm{pls}$.

Sowerby, J. De C., 1823-29, The mineral conchology of Great Britain: 1823 , v. 4 , p. $115-148$, pls. $384-407$, v. 5 , p. $1-64$, pls. $408-443 ; 1824$, v. 5, p. $65-138$, pls. $444-485 ; 1825$, v. 5 , p. $139-168$, pls. $486-503$; 1826 , v. 6 , p. $1-86$, pls. $504-$ 545 ; 1827 , v. 6 , p. $87-156$, pls. $546-580 ; 1828$, v. 6 , p. $157-$ 200 , pls. $581-597 ; 1929$, v. 6 , p. $201-230$, pls. $598-609$, London, B. Meredith.

Spieker, E. M., 1946, Late Mesozoic and early Cenozoic history of central Utah: U.S. Geol. Survey Prof. Paper 205-D, p. 117161, pls. 18-25, figs. 14-21.

Stanton, T. W., 1899, Mesozoic fossils of the Yellowstone National Park: U.S. Geol. Survey Mon. 32, pt. 2, p. 600-650, pls. $72-76$.

Veatch, A. C., 1907, Geography and geology of a portion of southwestern Wyoming: U.S. Geol. Survey Prof. Paper 56, 178 p., 26 pls., 9 text-figs.

Warren, P. S., 1932, A new pelecypod fauna from the Fernie formation, Alberta: Royal Soc. Canada Proc. and Trans., $3 \mathrm{~d}$ ser., v. 26 , sec. 4 , p. 1-36, pls. 1-5.

Wells, John W., 1942, A new species of coral from the Jurassic of Wyoming: Am. Mus. Nat. History, Am. Mus. Novitates no. 1161 , p. 1-3.

White, C. A., 1874, Preliminary report upon invertebrate fossils collected by expeditions of 1871,1872 , and 1873 , with descriptions of new species: U.S. Geog. and Geol. Surveys West 100th Meridian (Wheeler), $27 \mathrm{p}$.

- 1877, Report upon the invertebrate fossils collected in portions of Nevada, Utah, Colorado, New Mexico, and 
Arizona ***: U.S. Geog. Surveys West 100th Meridian (Wheeler), v. 4., pt. 1, 219 p., 21 pls.

1880, Contributions to invertebrate paleontology, Nos. 2-8: U.S. Geol. Survey Terr. (Hayden), 12th Ann. Rept., 1878, pt. 1, p. 5-171.

- 1884, Fossil Ostreidae of North America ***: U.S. Geol. Survey 4th Ann. Rept., p. 273-430.

Whiteaves, J. F., 1884, On the fossils of the coal-bearing deposits of the Queen Charlotte Islands collected by Dr. G. M. Dawson in 1878: Canada Geol. Survey, Mesozoic fossils, v. 1, pt. 3, p. 191-262, pls. 21-33.

1900 , On some additional or imperfectly understood fossils from the Cretaceous rocks of the Queen Charlotte Islands, with a revised list of the species from these rocks: Canada Geol. Survey, Mesozoic fossils, v. 1, pt. 4, p. 263307.

Whitfield, R. P., 1876, Descriptions of new species of fossils, in Ludlow, William, Report of a reconnaissance from Carroll, Montana Territory, on the upper Missouri, to the Yellowstone National Park and return, made in the summer of 1875: Washington, D.C., p. 141-145, also in U.S. [War Dept.], Chief Engineers, Ann. Rept. 1876 (U.S. 44th Cong. 2d sess., H. Exec. Doc. 1, pt. 2, v. 2, pt. 3), App. NN: p. 694-699.
1877, Preliminary report on the paleontology of the Black Hills, containing descriptions of new species of fossils from the Potsdam, Jurassic, and Cretaceous formations of the Black Hills of Dakota: U.S. Geog. and Geol. Survey Rocky Mtn. Region (Powell), 49 p.

_- 1880, Paleontology of the Black Hills of Dakota, in Newton, Henry, and Jenney, W. P., Report on the geology and resources of the Black Hills of Dakota: U.S. Geog. and Geol. Survey Rocky Mtn. Region (Powell), p. 329-468, pls. 1-15.

Whitfield, R. P., and Hovey, E. O., 1906, Remarks on and descriptions of Jurassic fossils of the Black Hills: Am. Mus. Nat. History Bull., v. 22, art. 23, p. 389-402, pls. 42-62.

Willard, M. E., and Callaghan, Eugene, 1962, Geology of the Marysvale quadrangle, Utah: U.S. Geol. Survey Geol. Quad. Map GQ-154, scale 1:62,500.

Wright, J. C., and Dickey, D. D., 1958, Pre-Morrison Jurassic strata of southeastern Utah; in Intermountain Association of Petroleum Geologists, Guidebook to the geology of the Paradox basin, 9th annual field conference 1958: Salt Lake City, Utah, p. 172-181.

___ 1963, Relations of the Navajo and Carmel Formations in southwestern Utah and adjoining Arizona: Art. 197 in U.S. Geol. Survey Prof. Paper 450-E, p. E63-E67. 


\section{INDEX}

[Italic page numbers indicate descriptions]

A

Page

acuticosta, Myopholas _......... C37

Ages and correlations ...........................

americana, Trigonia ${ }_{---}$4, 8, 9,10,12, 28, 29,30,32; pl. 3

Ammonites _.............. 4,10

Anatina punctata ........................... 37

(Cercomya) punctata .................... 37

semiradiata.............................- $\quad 37$

Anomia ............................. 2,26 sp_..................... 4, 10, 12, 26; pl. 1 Anomiidae.

Arapien Shale-..... 2, 3, 5,6

Arcticoceras _.............................. 29 codyense............................... 30

argo, Scaphogonia

Astarte .................... 2, 39

livingstonensis.............................. 10,34

meeki_............................... 4, 8,9,12

(Coelastarte)_............. 4, 8,12,22, $94 ;$ pl. 4 aytonensis......................... 33 interlineata.............. 4,6,34; pl. 4 livingstonensis.......... 3, 4,6,10,12, s9; pl. 4 rectangula

$\mathrm{sp}$ Astartidae................................. $\quad 2$ asteriscus, Pentacrinus. aytonensis, Astarte (Coelastarte)

bathonica, Quenstedtia _._. 4, 9,11,12,35; pl. 4 bellistriatus, Camptonectes.

Biologic analysis

Cadoceras

4,29

Camptonectes...................... 1, 2, 5, 7,22,23,25

bellistriatus. ................................ 26

distans

extenuatus..........................

platessiformis . ............ 4,6,8,9,10,12,26; pl. 2 stygius_.............. 4, $6,8,9,10,11,12,25 ; \mathrm{pl} .2$ sp. ................................ $6,8,9,10,11,12$

Carmel Formation.

Cercomya............................... 2, 97 punctata semiradiata._. striata.

(Cercomya) punctata, Anatina semiradiata, Anatina.................... 37

Chondroceras .................................. 3

cinnabarensis, Cyprina Pronoella........ 4, 6, 8, 9, 10,11,12, 84,$35 ;$ pl. 4

codyense, Arcticoceras...................... 30

Coelastarte ............................ 2, 38,34 livingstonensis (Coelastarte)

(Coelastarte), Astarte aytonensis, Astarte..................... interlineata, Astarte.......... 4, 6,34; pl. 4 livingstonensis, Astarte..... 3,4,6,10,12,99; pl. 4 rectangula, Astarte..................... 33 Coelastarte

Colonial corals

conradi, Myophorella (Promyophorella)

Trigonia

Vaugonia ........ 4, 6,8,9,10,11, 12,30,31; pl. 3

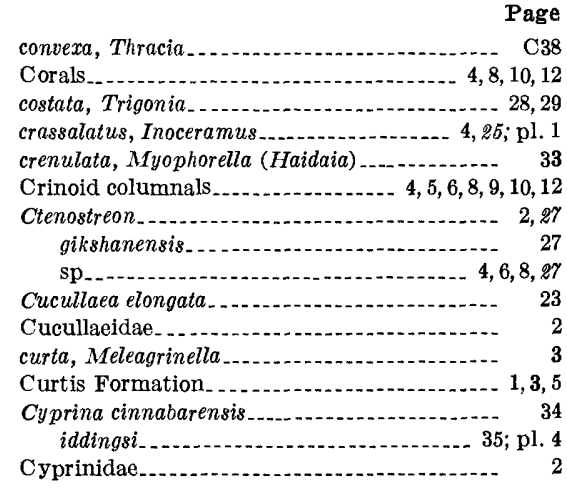

D

dawsoni, Myophorella (Haidai) ................ 33 denticulata, Trigonia._....................... 28

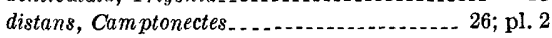
dowlingi, Ostrea

\section{E}

Echinoid fragments........ 4, 5, 6, 8, 9, 12 elegantissima, Trigonia_..... 4, 8, 9, 10,11, 12, 29; pl. 3 elongata, Cucullaea engelmanni, Lopha................ 4, 8,9,12,28; pl. 1 Ostrea Entrada Sandstone............................ 5 Eocallista extenuatus, Camptonectes................... 26

\section{F}

Faunal associations and comparisons ......... 6 ferrieri, Trigonia.............................. 32

\section{G}

Gastropods $\ldots \ldots \ldots \ldots, \ldots, \ldots, 9,10,11,12$ geniculata, Vaugonia (Hijitrigonia)

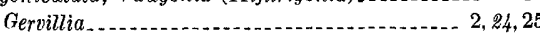
montanaensis _............ 3,4,5,9,10,12,24; pl. 1 sparsilirata .......................... 24; pl. 1 (Gervillia) perplana, Myalina........ gikshanensis, Ctenostreon ...................... 27

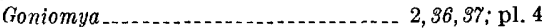
hemicostata $\ldots . . . \ldots \ldots$ montanaensis . ........... 3, $4, \mathbf{1 0}, \mathbf{3 6}, \mathbf{3 7} ; \mathrm{pl} .4$ normanniana....................... 37

Grammatodon

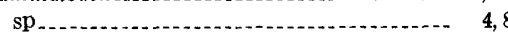

Gryphaea

planoconvexa................................ 24

guhsani, Trigonia................. 28

H

haguei, Idonearca............................ 23 Haidaia.......................................... 2, 23

(Haidaia) crenulata, Myophorella............- 33 dawsoni, Myophorella................... 33 yellowstonensis, Myophorella _... 3, 4, 10,33; pl. 3 Hamulus subquadratus.................. 2, 10; pl. 4 hardyi, Myopholas...................... 4, 6, 37; pl. 4

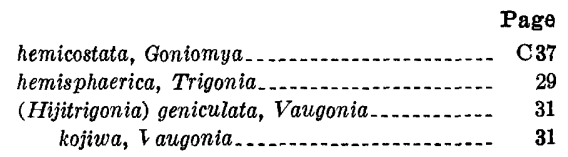

iddingsi, Cyprina........................ 35; pl. 4 Idonearca haguei.................................. 23 sp $\ldots \ldots \ldots \ldots \ldots \ldots$ imbricatus, Modiolus.......................... 23 inaequiplicata, Pholadomya....- 4, 6, 9, 10,12,36; pl. 4 Inoceramus................................ 2, 25 crassalatus_..................... 4, 25; pl. 1 interlineata, Astarte (Coelastarte) _....... 4,6,34; pl. 4 Introduction.

Isocyprina

Isognomon ............................... 2, 7, 22, 24,25 perplana promytiloides............................... 24 weelaupensis............................... 24

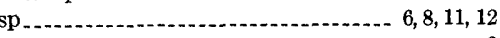
Isognomonidae ................................ 2 isonema, $V$ olsella scalprum............... isonemus, Modiolus..................... 4, 6, 24; pl. 1

$\mathrm{J}$

jurassica, Pinna............................. 25

K

Kepplerites.

kingi, Pholadomya................... 4,6,10,36; pl. 4 Pinna.............................. 4, 12,25 kojiwa, Vaugonia (Hijitrigonia)

\section{$\mathbf{L}$}

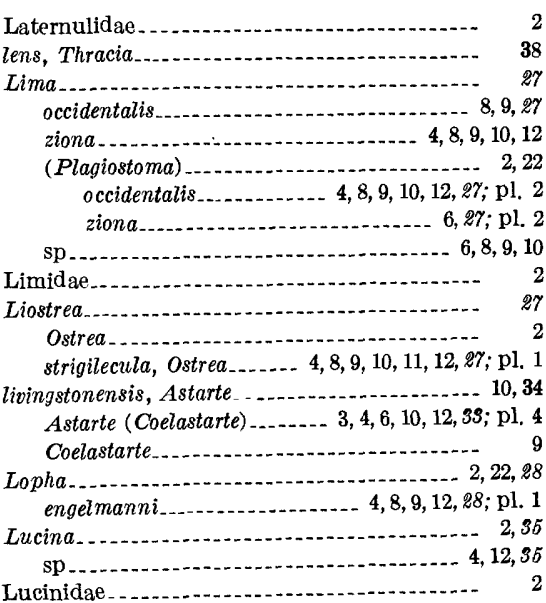

$\mathrm{M}$

Mactromya .

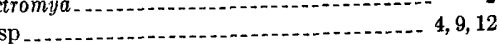
mariajosephinae, Vaugonia................... $\quad 30$ meeki, Astarte. $4,8,9,12$ 


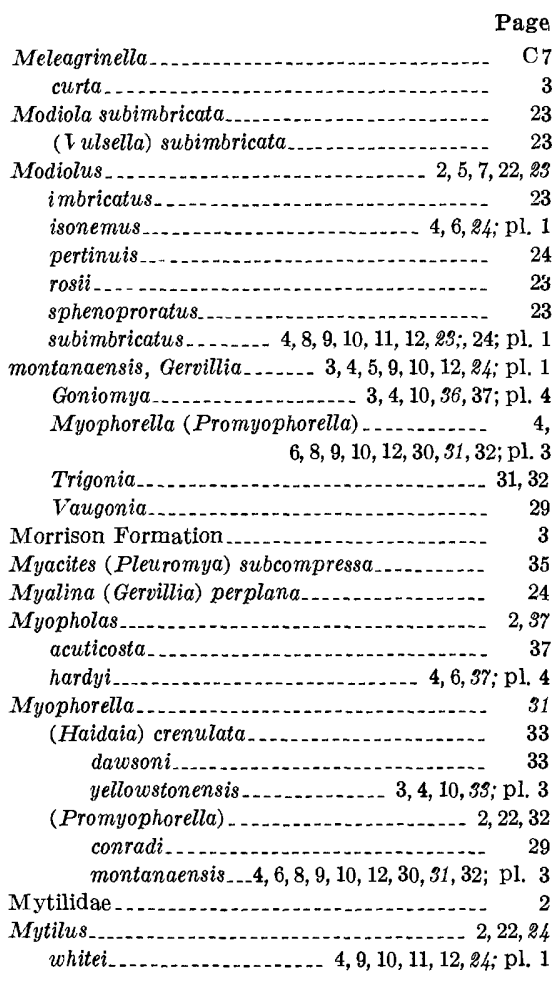

$\mathrm{N}$

Navajo Sandstone................................ navis, Trigonia

Nerineid gastropods........................ 4 newtoni, Pleuromya normanniana, Goniomya.................... 37 Nucula sp $\ldots . . \ldots$ 4, 8,$29 ; \mathrm{pl} 1$ Nuculidae...................

Nugget Sandstone............................... 6

occidentalis, Lima............................. 8,9,27 Lima (Plagiostoma) ........ 4, 8, 9, 10, 12, 27; pl. 2

Ostrea ............................ 1,2, 5, 7,22, 27 dowlingi................................ 28 engelmanni.............................. 28 strigilecula _.......................... 6, 27, 28

(Liostrea) strigilecula......... 4, 8,9,10,11, 12, 27; pl. 1 Ostreidae

Parallelodontidae

Pectinid

Pentacrinus asteriscus

peregrina, Plicatula

Perna -

perplana, Isognomon_._._ 4, 8, 9, 10,11, 12, 24, 25; pl. 1 Myalina (Gervillia) .................... 24 pertinuis, Modiolus ..................... 24 Pholadidae.......................................

Pholadomya............................... 22, 36 inaequiplicata ............ 4, 6, 9, 10, 12, 36; pl. 4 kingi.......................... 4, 6, 10, $36 ; \mathrm{pl} .4$ sp

Pholadomyidae

Pinna_............................... 2, 25 jurassica

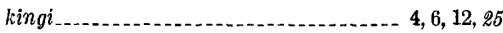
sp $\ldots \ldots \ldots$

Pinnidae.

7

3

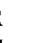

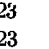

\section{.}

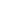

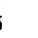
4 


\section{PLATES 1-4}




\section{PLATE 1}

[Figures natural size unless otherwise indicated]

Figures 1-3. Nucula sp. (p. C23).

Figured specimens USNM 131917 from USGS Mesozoic loc. 19432.

4-6. Mytilus ef. M. whitei Whitfield (p. C24).

4. Lateral view of figured specimen USNM 131925 from USGS Mesozoic loc. 15551.

5, 6. Lateral and dorsal views of figured specimen USMN 131924 from USGS Mesozoic loc. 6941.

7-9. Lopha off. L. engelmanni (Meek) (p. C28).

7. Figured specimen USNM 131954 from USGS Mesozoic loc. 17353.

8, 9. Figured specimen USNM 131955 from USGS Mesozoic loc. 5263.

10-12. Idonearca? sp. (p. C23).

Figured specimen USNM 131918 from USGS Mesozoic loc. 26307. Fig. 2 shows internal cast of left valve.

13-15. Plicatula sp. (p. C26).

13, 14. Finely ribbed specimens USNM 131941 from USGS Mesozoic loc. 13756.

15. Coarsely ribbed specimen USNM 131942 from USGS Mesozoic loc. 12555.

16-20. Modiolus subimbricatus (Meek) (p. C23).

16. Syntype USNM 131920 from Lower Canyon of the Yellowstone River, Montana.

17. Plesiotype USNM 131922 from USGS Mesozoic loc. 17054.

18. Lectotype USNM 131919 from Lower Canyon of the Yellowstone River, Montana.

19. Syntype USNM 131921 from Lower Canyon of the Yellowstone River, Montana.

20. Plesiotype USNM 131923 from USGS Mesozoic loc. 16624.

21. Gervillia sparsilirata Whitfield (p. C24).

Lateral view of plaster replica of holotype 21281 in the Yale Peabody Museum. This species is a synonym of $G$. montanaensis Meek.

22. Modiolus isonemus (Meek) (p. C24).

Holotype USNM 12545 from Weber Canyon, Utah.

23. Anomia? sp. (p. C26).

Figured specimen USNM 131943 from USGS Mesozoic loc. 18922.

24,25 . Inoceramus crassalatus White (p. C25).

24. Syntype USNM 131930 from North Fork of Virgin River, Utah.

25. Lectotype USNM 131929 from North Fork of Virgin River, Utah.

26-29. Ostrea (Liostrea) strigilecula White (p. C27).

26. Left valve of plesiotype USNM 131953 from USGS Mesozoic loc. 16202.

27. Left valve of lectotype USNM 131951 from south of Dirty Devil River, Utah.

28. Interior of right valve of syntype USNM 8581 from same locality as the lectotype.

29. Interior of right valve of syntype USNM 131952 from same locality as the lectotype.

30, 31, 35, 36. Isognomon ef. I. perplana (Whitfield) (p. C25).

30, 31. Internal casts showing ligamental areas of figured specimens USNM 131929 from USGS Mesozoic loc. 16624 .

35. Internal mold of right valve of figured specimen USNM 131928 from USGS Mesozoic loc. 963.

36. Left valve of internal mold assigned by White to Mytilus whitei Whitfield. Figured specimen USNM 8177 from near Fontenelle Canyon on west side of Green River Basin, Wyoming.

32-34. Isognomon perplana (Whitfield) (p. C24).

32. Right valve of plesiotype USNM 131926 from USGS Mesozoic loc. 1158 in Yellowstone National Park.

33. Left valve of holotype 21280 in the Yale Peabody Museum from the south end of the Bridger Range,

Montana.

34. Left valve of plesiotype USNM 131927 from USGS Mesozoic loc. 1158 in Yellowstone National Park. 
GEOLOGICAL SURVEY
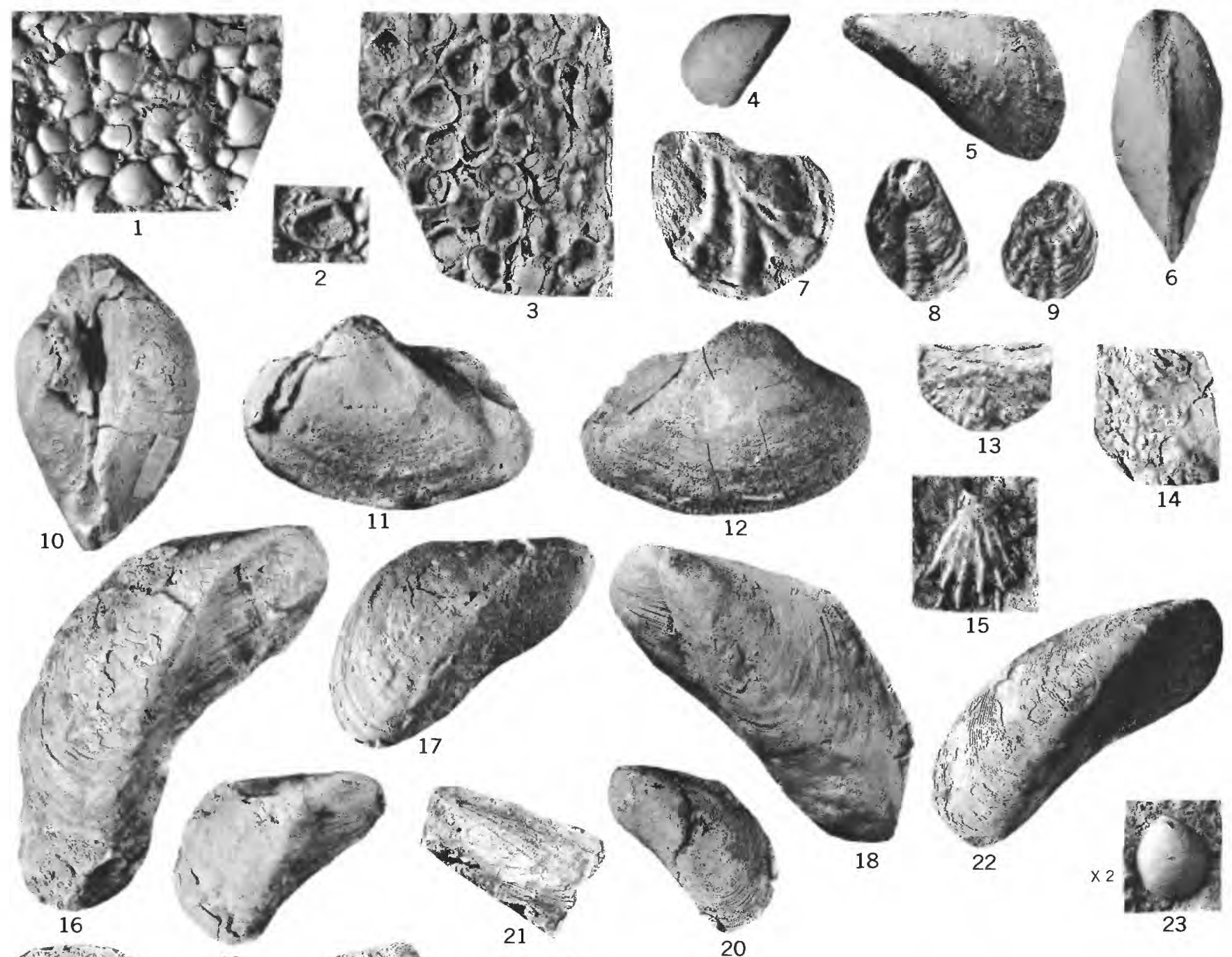

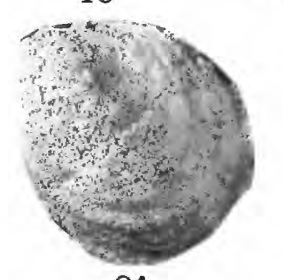

24
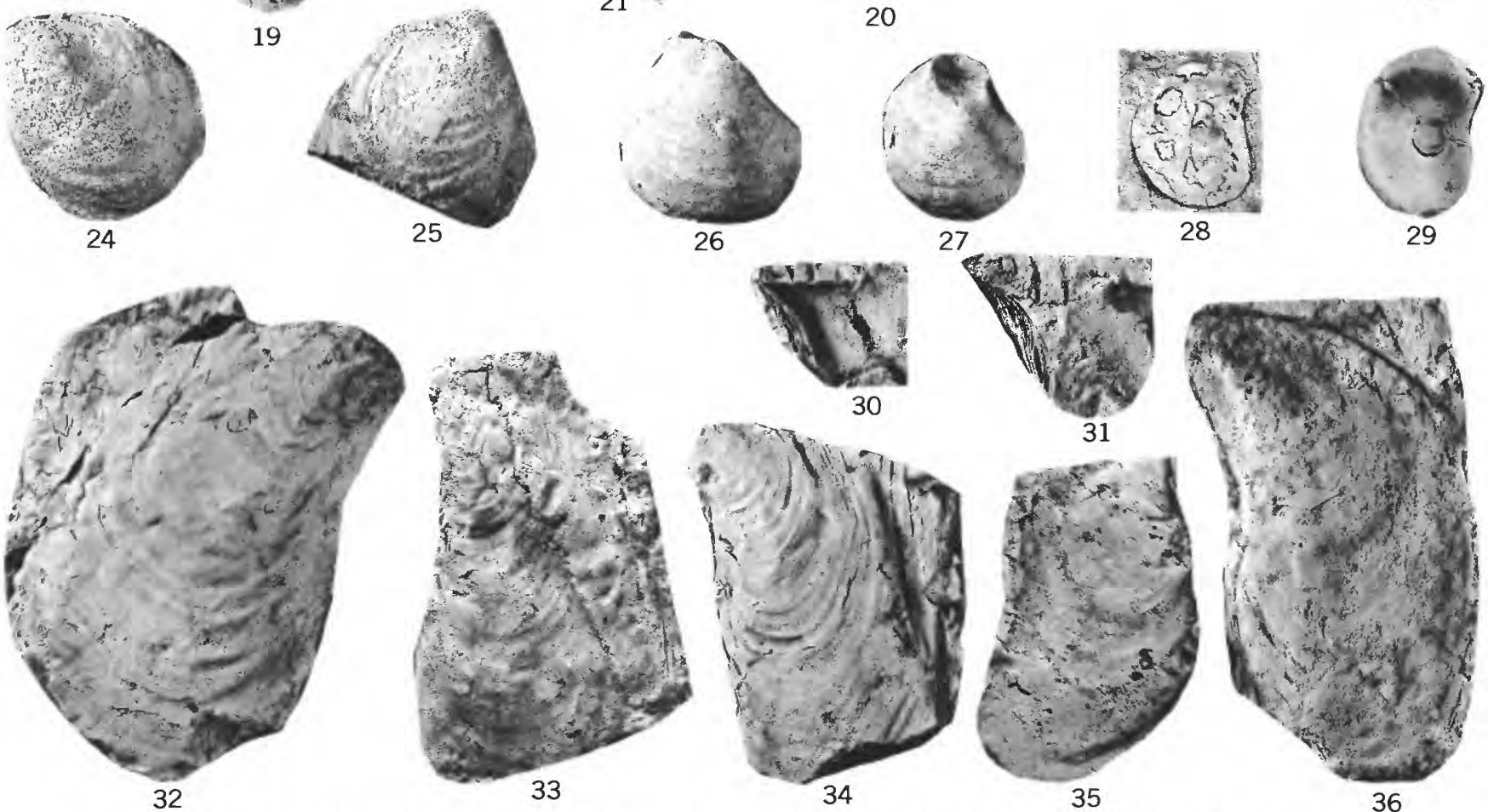

NUCULA, MYTILUS, LOPHA, IDONEARCA ?, PLICATULA, MODIOLUS, ANOMIA?, GERVILLIA, INOCERAMUS, OSTREA (LIOSTREA), AND ISOGNOMON 


\section{PLATE 2}

[Figures natural size unless otherwise indicated]

Figures 1-10. Camptonectes stygius White (p. C25).

1. Right valve of lectotype, USNM 131931 from southern Utah.

2. Interior of right valve of syntype USNM 8580 from the type lot.

3. Right valve of syntype USNM 131932 from the type lot.

4. Small right valve of syntype USNM 131934 from the type lot.

5. Small right valve of syntype USNM 131933 from the type lot.

6. Right valve of plesiotype USNM 131936 from USGS Mesozoic loc. 17353.

7. Right valve of plesiotype USNM 131937 from USGS Mesozoic loc. 10436.

8, 9. Left valve and posterior view of plesiotype USNM 131935 from USGS Mexozoic loc. 18281.

10. Left valve of plesiotype USNM 131938 from USGS Mesozoic loc. 10436.

11-14. Camptonectes platessiformis White (p. C26).

11, 12. Left valve of holotype USNM 8179 from north base of Aquarius Plateau, Utah. Fig. 11 is shown $\times 2$.

13. Left valve of plesiotype USNM $131940(\times 2)$ from USGS Mesozoic loc. 16624.

14. Left valve of plesiotype USNM $131939(\times 2)$ from USGS Mesozoic loc. 25669.

15, 16. Camptonectes distans Stanton (p. C26).

Right valve of holotype USNM 28933 from USGS Mesozoic loc. 1158 in Yellowstone National Park.

17-20. Lima (Plagiostoma) occidentalis Hall and Whitfield (p. C27).

17. Left valve of plesiotype USNM 131946 from USGS Mesozoic loc. 26309.

18. Left valve of plesiotype USNM 131945 from USGS Mesozoic loc. 27474.

19. Right valve of plesiotype USNM 131944 from USGS Mesozoic loc. 16624.

20. Left valve of holotype USNM 12539 from the Flaming Gorge near Manila, Utah.

21-26. Lima (Plagiostoma) ziona Imlay, n. sp. (p. C27).

21. Small left valve of paratype USNM 131950 from USGS Mesozoic loc. 25675.

22-24. Posterior view, left valve and right valve of holotype USNM 131947 from USGS Mesozoic loc. 12749.

25. Small right valve of paratype USNM 131949 from USGS Mesozoic loc. 25675.

26. Complete large left valve of paratype USNM 131948 from USGS Mesozoic loc. 27469. 
GEOLOGICAL SURVEY
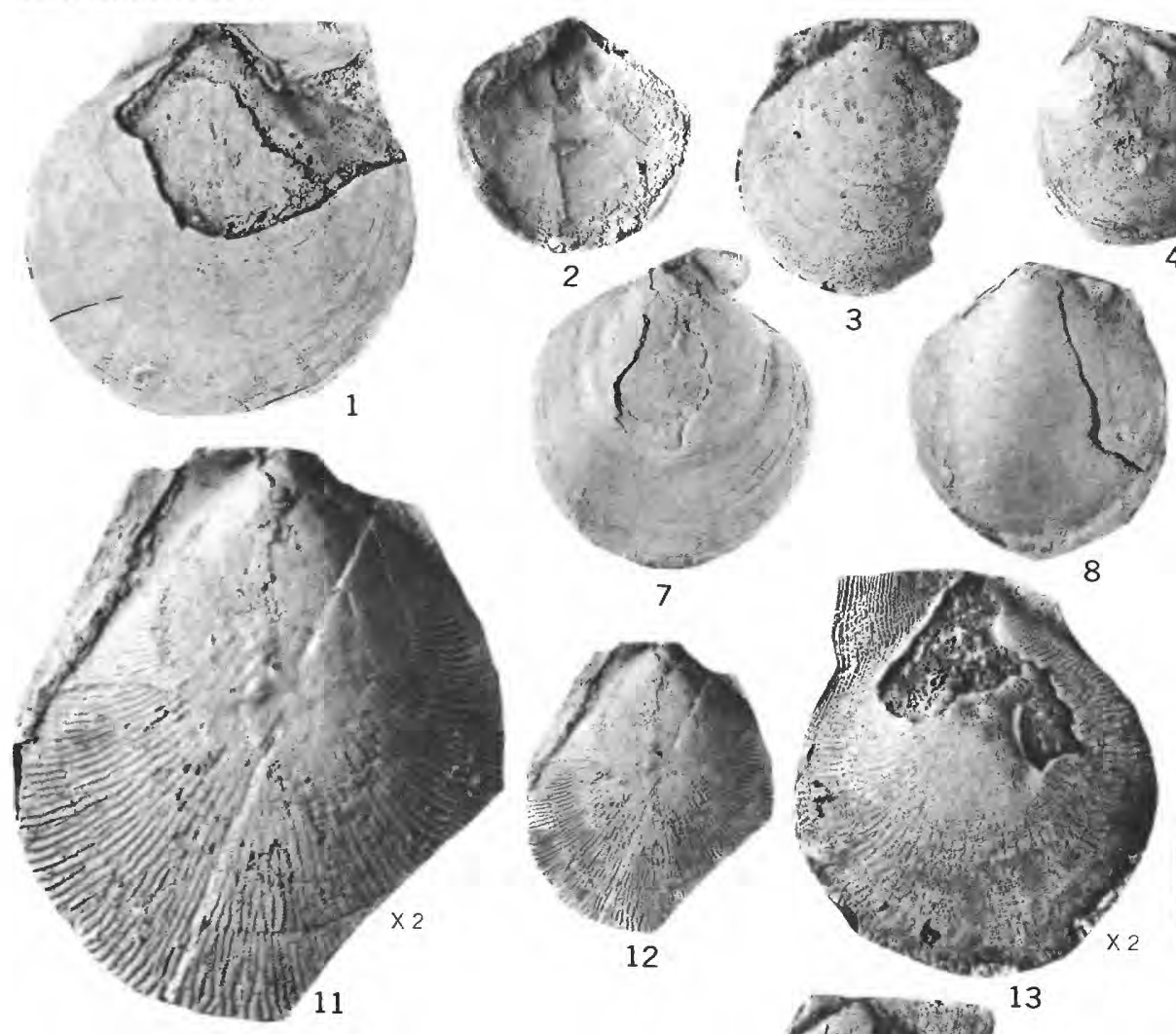

12
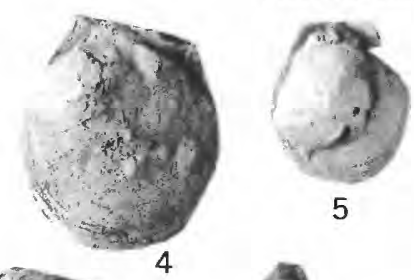

5
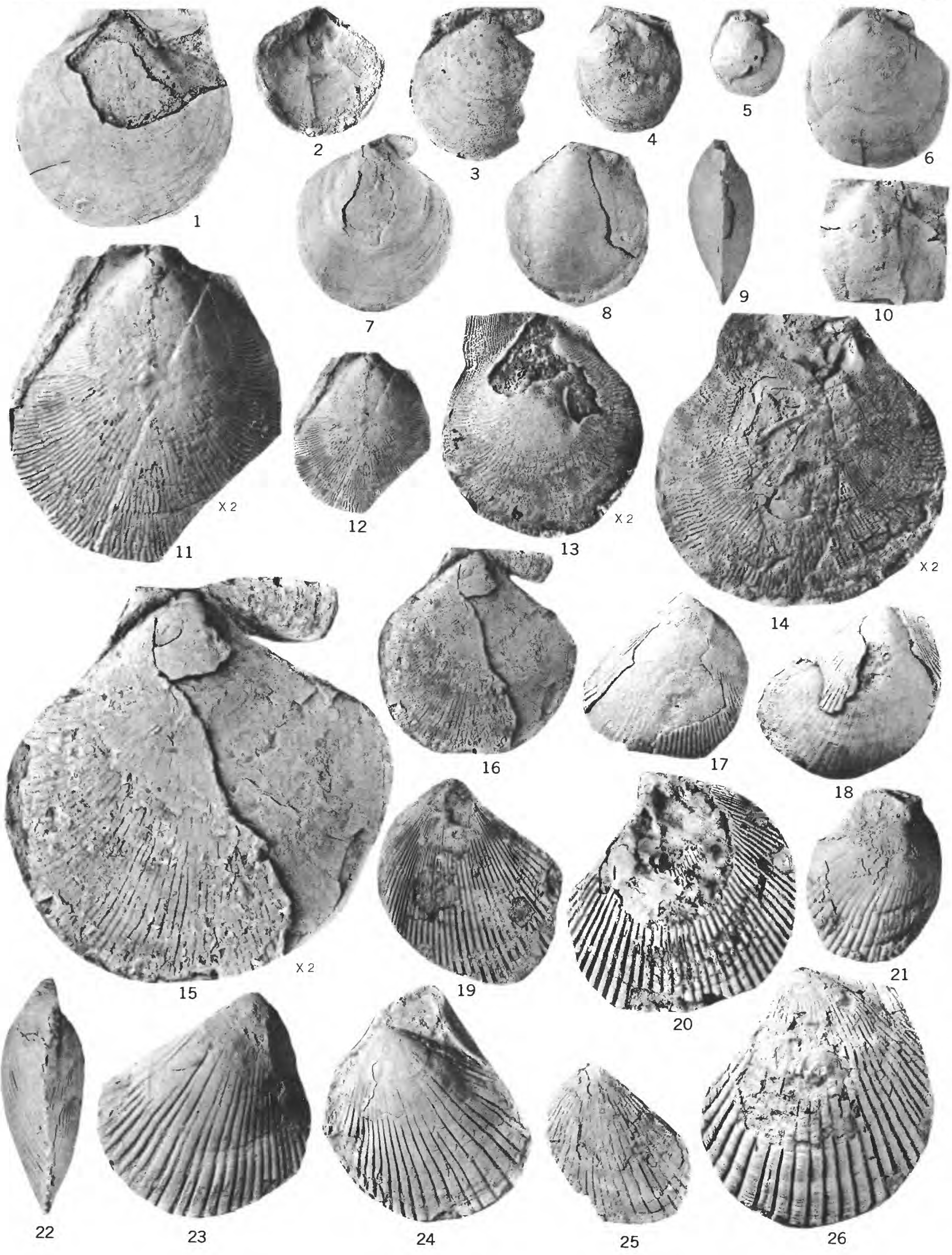

CAMPTONECTES AND LIMA 


\section{PLATE 3}

[All figures are natural size]

Figures 1-7. Trigonia elegantissima Meek (p. C29).

1. Coarsely ribbed right valve of plesiotype USNM 131963 from USGS Mesozoic loc. 11439.

2. Finely ribbed left valve of plesiotype USNM 28935 from USGS Mesozoic loc. 1182, Cinnabar Mountain, Montana. This specimen was illustrated by Stanton.

3. Coarsely ribbed left valve of plesiotype USNM 131964 from USGS Mesozoic loc. 25686.

4. Moderately ribbed plesiotype USNM 131960 from USGS Mesozoic loc. 20974, Lower Slide Lake, Teton County, Wyo.

5. Plesiotype USNM 131959 from USGS Mesozoic loc. 20974, Lower Slide Lake, Wyoming.

6. Moderately ribbed plesiotype USNM 131961 from USGS Mesozoic loc. 19422.

7. Finely ribbed plesiotype USN M 131962 from USGS Mesozoic loc. 19422.

8-13. Trigonia americana Meek (p. C28).

8, 13. Dorsal and lateral views of right valve of plesiotype USNM 131958 from USGS Mesozoic loc. 25866 in sec. 35 , T. 1 S., R. 9 E., Park County, Mont.

9. Lectotype USNM 131956 from mouth of Yellowstone Canyon near Livingston, Mont.

10. Syntype USNM 7794 from same locality as lectotype.

11. Syntype USNM 7819 from same locality as lectotype.

12. Syntype USNM 131957 from same locality as lectotype.

14-21. Vaugonia conradi (Meek and Hayden) (p. C30).

14. Plesiotype USNM 131965 from USGS Mesozoic loc. 20362 near Cody, Wyo.

15. Holotype USNM 212 from the southwest base of the Black Hills, Wyoming. The specimen is corroded and lacks posterior end but is comparable to specimen shown in fig. 14 .

16. Corroded right valve of plesiotype USNM 131968 from USGS Mesozoic loc. 25669.

17. Corroded right valve of plesiotype USNM 131966 from USGS Mesozoic loc. 26309.

18. Corroded right valve of plesiotype USNM 131969 from USGS Mesozoic loc. 25669.

19. Corroded right valve of plesiotype USNM 131967 from USGS Mesozoic loc. 25686.

20. Left valve of plesiotype USNM 131971 from USGS Mesozoic loc. 20958 from Lower Slide Lake, Teton County, Wyo.

21. Right valve of plesiotype USNM 131970 from USGS Mesozoic loc. 20958, Wyoming. Compare sharpness of ribbing in internal molds shown in figs. 20 and 21 with corroded internal molds shown in figs. 15-19.

22. Vaugonia quadrangularis (Hall and Whitfield) (p. C30).

Holotype USNM 12537 from Como Bluff, Wyo. Photograph made from rubber cast of an external mold that lacks part of anterior end.

23-26. Vaugonia utahensis Imlay, n. sp. (p. C31).

23, 24. Dorsal and lateral views of right valve of holotype USNM 131973 from USGS Mesozoic loc. 26309.

25. Left valve of paratype USNM 131975 from USGS Mesozoic loc. 27473.

26. Right valve of paratype USNM 131974 from USGS Mesozoic loc. 27473.

27-32. Myophorella (Haidaia) yellowstonensis Imlay, n. sp. (p. C33).

27. Right valve of holotype USNM 131983 from USGS Mesozoic loc. 27624, near East Boulder River, Montana, sec. 23, T. 3 S., R. 12 W., about $165 \mathrm{ft}$ below top of Piper Formation.

28. Paratype USNM 131984 from USGS Mesozoic loc. 27624, Montana.

29. Paratype USNM 131985 from USGS Mesozoic loc. 13531, Utah.

30. Paratype USNM 131987 from USGS Mesozoic loc. 20967, Lower Slide Lake, Teton County, Wyo., from unit 10 of published section (Imlay, 1956a, p. 70).

31. Paratype USNM 131988 from USGS Mesozoic loc. 20967, Wyoming.

32. Paratype USNM 131986 from USGS Mesozoic loc. 21385, Madison Range, NE1/4 SE1/4 sec. 35, T. 5 S., R. 1 E., Montana, Sawtooth Formation.

33-36, 38-41. Myophorella (Promyophorella) montanaensis (Meek) (p. C31).

33. Plesiotype USNM 131978 from USGS Mesozoic loc. 13532.

34. Plesiotype USNM 131977 from USGS Mesozoic loc. 27628, 21/2 miles south of Livingston, Mont.

35. Plesiotype USNM 131979 from USGS Mesozoic loc. 6941.

36. Plesiotype USNM 131981 from USGS Mesozoic loc. 19622, 21/2 miles south of Livingston, Mont.

38. Lectotype USNM 131976 from Lower Canyon of the Yellowstone River, Montana.

39. Syntype USNM 7817 from same locality as the lectotype.

40. Plesiotype USNM 131982 from USGS Mesozoic loc. 12842.

41. Plesiotype USNM 131980 from USGS Mesozoic loc. 6941.

37. Vaugonia aff. V. conradi (Meek and Hayden) (p. C31).

Figured specimen USNM 131972 from USGS Mesozoic loc. 13529. 
GEOLOGICAL SURVEY
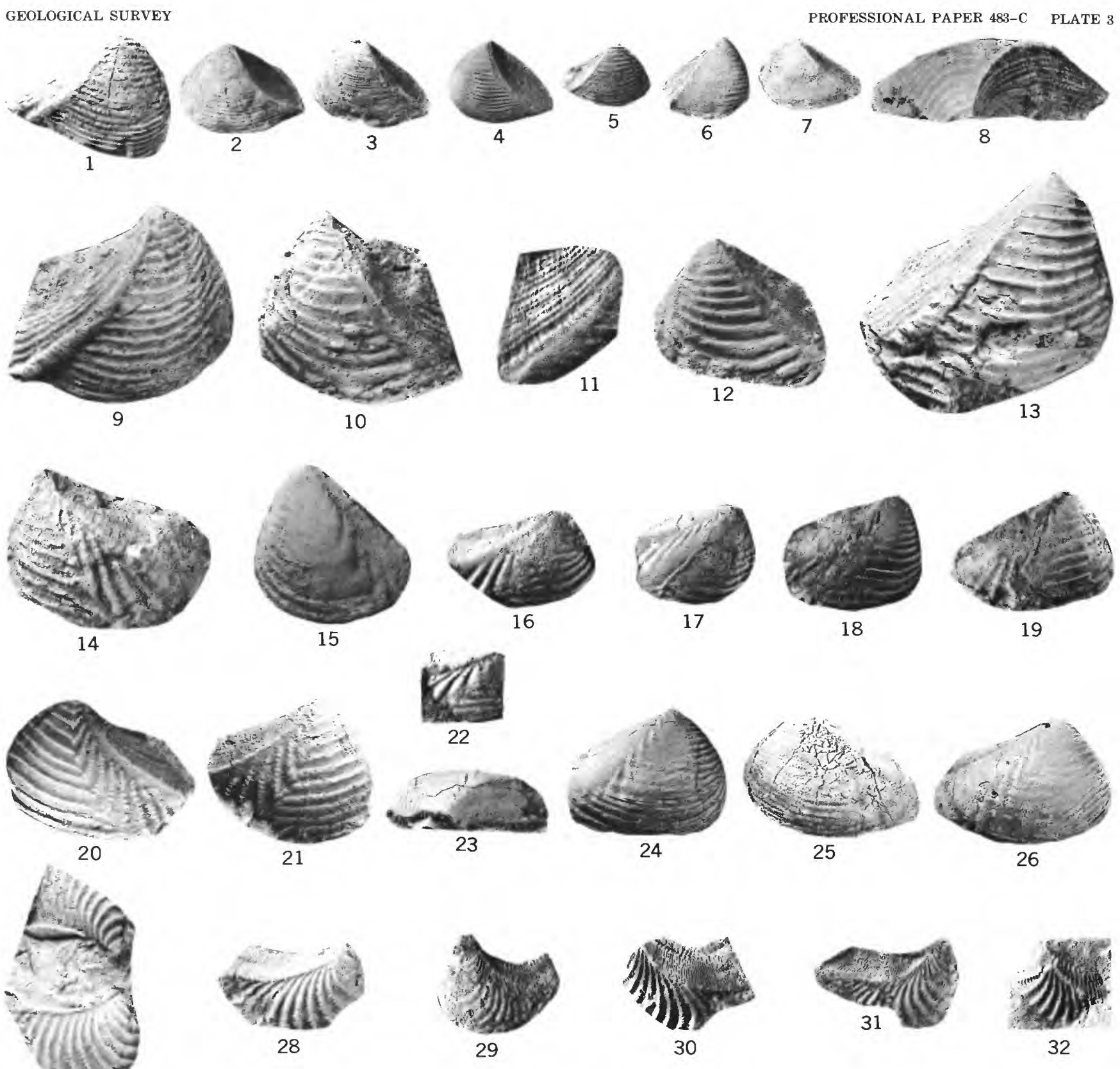
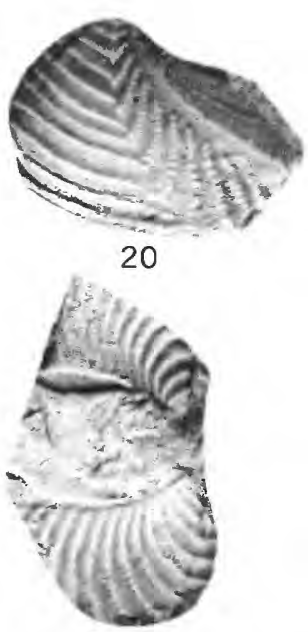

27
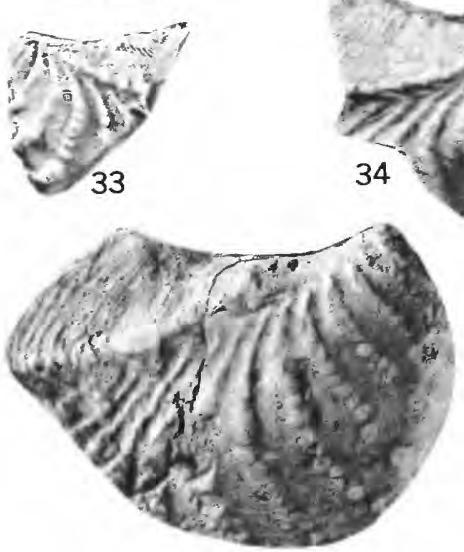

38
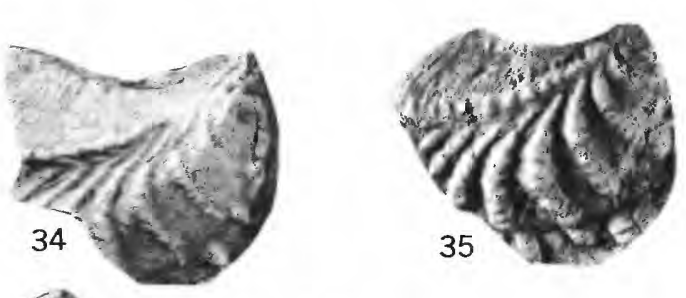
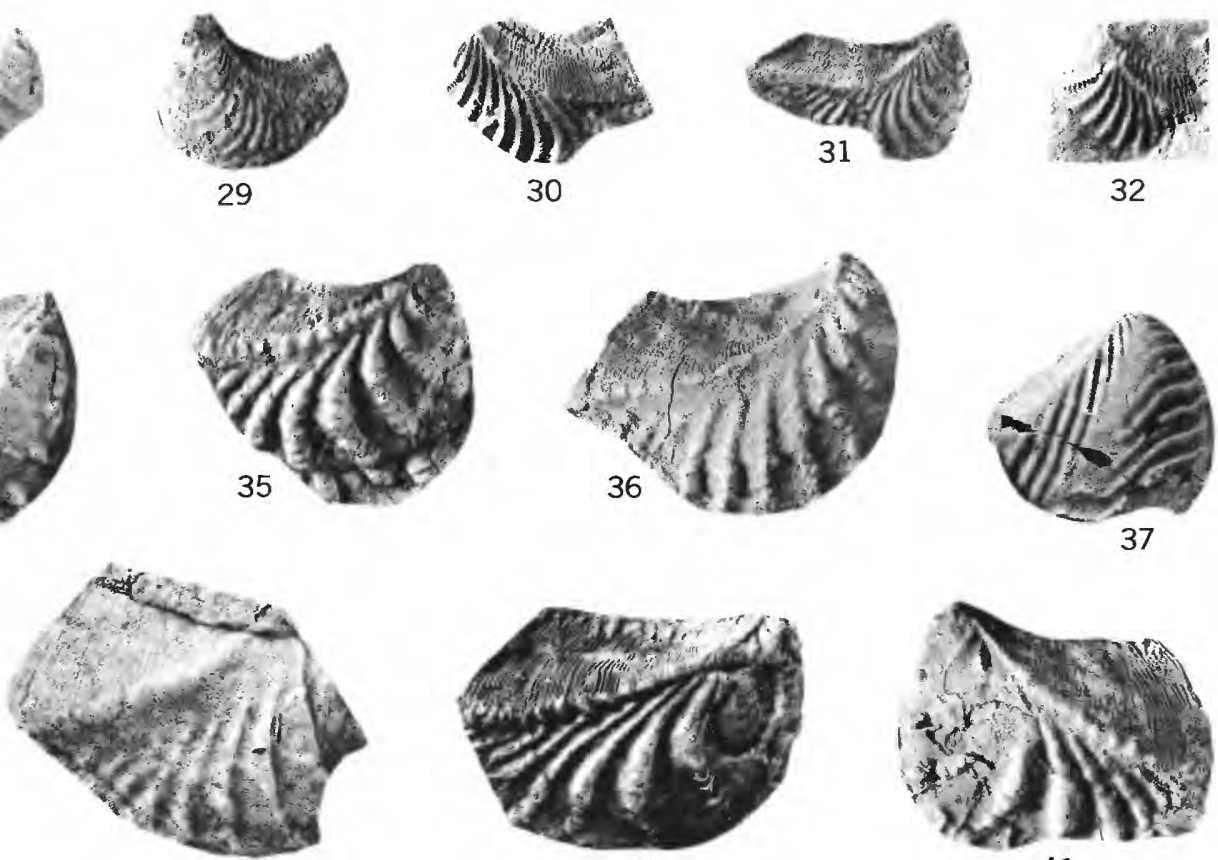

39

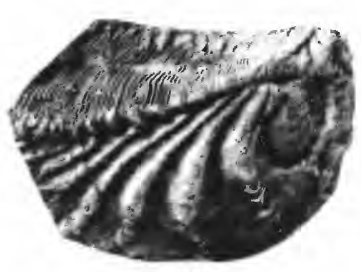

40

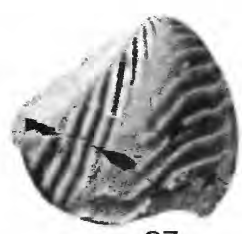

37

TRIGONIA, VAUGONIA, MYOPHORELLA (HAIDAIA), AND MYOPHORELLA (PROMYOPHORELLA) 


\section{PLATE 4}

[Figures natural size unless otherwise indicated]

Figures 1-4. Astarte (Coelastarte) livingstonensis Imlay, n. sp. (p. C33).

1. Holotype USNM 131989 from USGS Mesozoic loc. 25866 in sec. 35, T. 1 S., R. 9 E., Park County, Mont.

2. Paratype CSNM 12220 from Lower Canyon of Yellowstone River near Livingston, Mont.

3. Paratype USNM 131990 from USGS Mesozoic loc. 21385 in NE1/4SW1/4 sec. 35, T. 5 S., R. 1 E., Montana.

4. Paratype USNM 131991 from USGS Mesozoic loc. 25866, Park County, Mont.

5. Astarte (Coelastarte) ef. A. (C.) interlineata Lycett (p. C34).

Figured specimen USNM 131992 from USGS Mesozoic loc. 17269.

6-8. Astarte (Coelastarte) n. sp. undet. (p. C34).

6. Figured specimen USNM 132011 from CSGS Mesozoic loc. 28494.

7. Figured specimen USNM 131993 from USGS Mesozoic loc. 17269.

8. Figured specimen USNM 132012 from CSGS Mesozoic loc. 28495.

9-11. Quenstedtia aff. Q. bathonica Morris and Lycett (p. C35).

9. Specimen USNM 132000 from USGS Mesozoic loc. 17494.

10. Specimen USNM 132001 from USGS Mesozoic loc. 16701.

11. Specimen USNM 132002 from USGS Mesozoic loc. 12835.

12-14. Myopholas hardyi Imlay, n. sp. (p. C37).

12. View of rubber cast of external mold of paratype USNM 132008 from USGS Mesozoic loc. 21449.

13, 14. View of rubber cast of external mold of holotype USNM 132007 (X 1 and X 2) from USGS Mesozoic loc. 21448.

15-23. Pronoella uintahensis Imlay, n. sp. (p. C34).

15, 16. External and internal views of right valve of silicified holotype USNM 131994 from USGS Mesozoic loc. 20536 in northeast Utah.

17. Internal cast of paratype USNM 131996 from USGS Mesozoic loc. 16624.

18. Silicified paratype USNM 131998 from USGS Mesozoic loc. 25685.

19. Silicified paratype USNM 131999 from USGS Mesozoic loc. 25685.

20-22. Posterior, lateral and anterior views of paratype USNM 131997 from USGS Mesozoic loc. 19422.

23. Silicified paratype USNM 131995 from USGS Mesozoic loc. 20356 in northeast Utah. Shows hinge features.

24, 25. Cyprina? iddingsi Stanton (p. C35).

24. Internal cast of paratype CSNM 30653 from USGS Mesozoic loc. 1160 in Yellowstone National Park.

25. Internal mold of holotype USNM 28940 from USGS Mesozoic loc. 1173 in Yellowstone National Park.

26. Hamulus? subquadratus (Meek and Hayden).

Plesiotype USNM 132013 from USGS Mesozoic loc. 12555.

27-30. Pronoella cinnabarensis (Stanton) (p. C34).

27-29. Lateral and ventral views of lectotype USNM 28939 from USGS Mesozoic loc. 1168 in Yellowstone National Park.

30. Rubber cast of internal mold of syntype USNM 30648 from USGS Mesozoic loc. 1187 in Yellowstone National Park.

31. Cercomya punctata (Stanton) (p. C37).

Internal mold ef. plesiotype USNM 132009 from USGS Mesozoic loc. 26307.

32, 33. Thracia weedi Stanton (p. C38).

32. Left valve of lectotype USNM 28944 from USGS Mesozoic loc. 1145 in Yellowstone National Park.

33. Left valve of plesiotype USNM 132010 from USGS Mesozoic loc. 16701.

34, 35. Goniomya montanaensis Meek (p. C36).

Dorsal views of holotype CSNM 7814 shown under different lighting, from Lower Canyon of the Yellowstone

River near Livingston, Mont.

36. Pholadomya kingi Meek (p. C36).

Left valve of plesiotype USNM 132003 from USGS Mesozoic loc. 6941.

37, 38. Pholadomya inaequiplicata Stanton (p. C36).

37. Plesiotype USNM 132005 from USGS Mesozoic loc. 6941.

38. Plesiotype CSNM 132004 from USGS Mesozoic loc. 6941.

39. Goniomya? n. sp. undet. (p. C37).

Specimen USNM 132006 from USGS Mesozoic loc. 6944. 


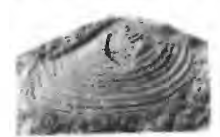

1

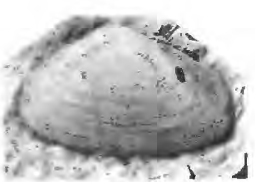

9

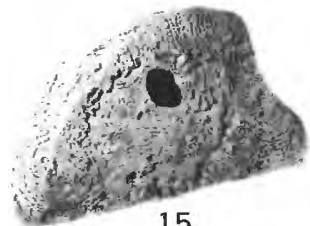

15

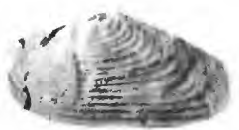

2

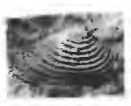

3

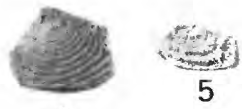

4

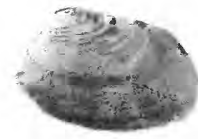

6

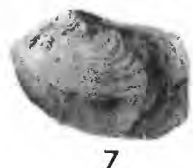

7

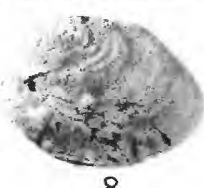

8

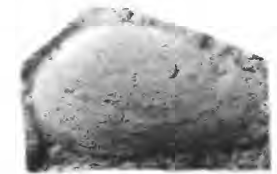

10

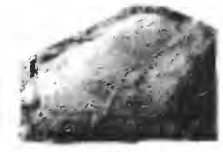

11

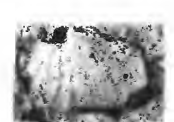

12
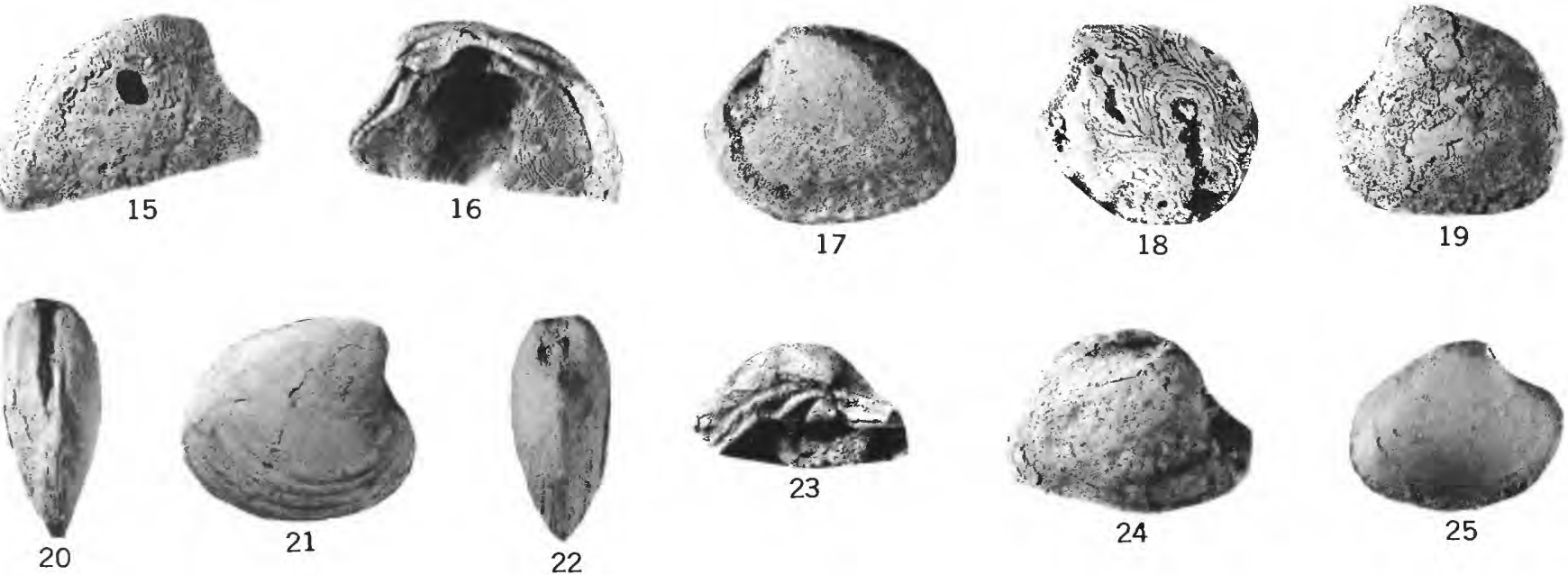

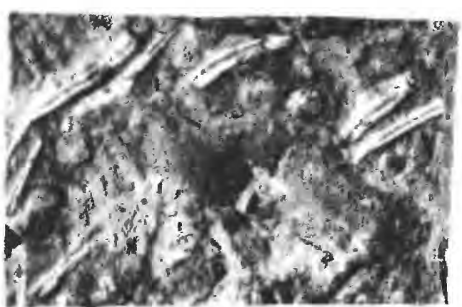

26

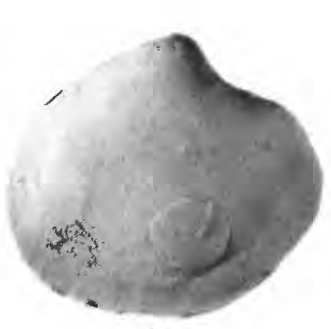

27

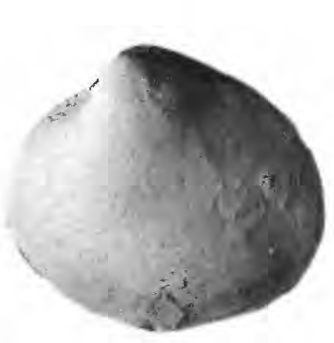

28

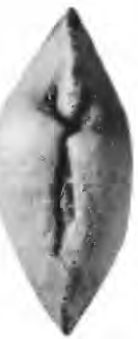

29

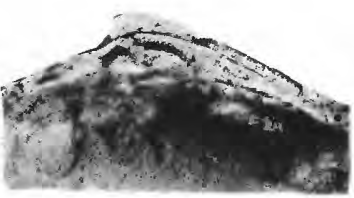

30

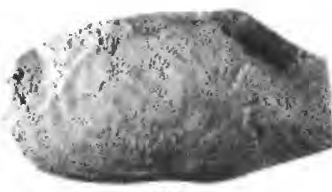

31

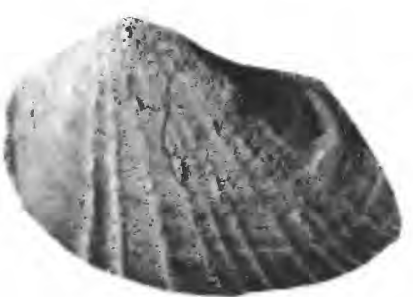

36

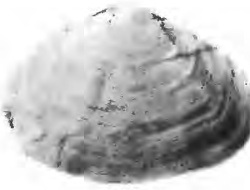

32

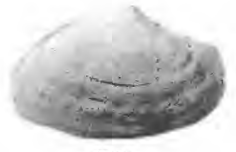

33
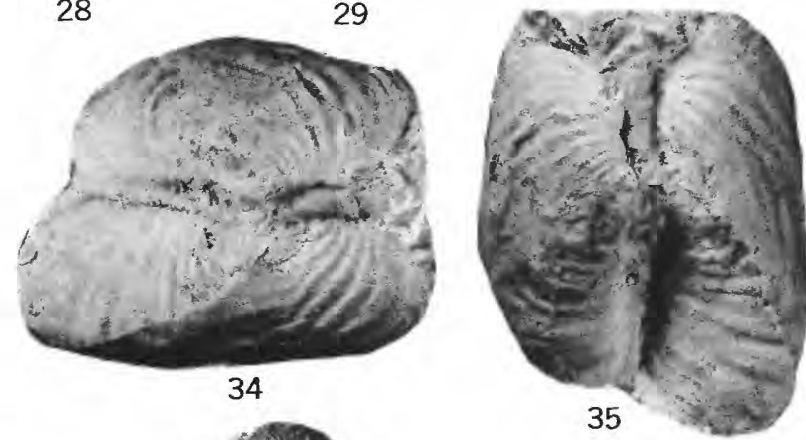

ASTARTE (COELASTARTE), QUENSTEDTTA, MYOPHOLAS, PRONOELLA, CYPRINA?, HAMULUS?, CERCOMYA, THRACIA, GONIOMYA, AND PHOLADOMYA 